Article

\title{
Decision Making Approach under Pythagorean Dombi Fuzzy Graphs for Selection of Leading Textile Industry
}

\author{
Muhammad Akram ${ }^{1, *(\mathbb{D}}$, Jawaria Mohsan Dar ${ }^{1}$ and Sundas Shahzadi ${ }^{2}$ \\ 1 Department of Mathematics, University of the Punjab, New Campus, Lahore 4590, Pakistan; \\ jiadar4@gmail.com \\ 2 Department of Mathematics, University of Education, Lower Mall Campus, Lahore, Pakistan; \\ sundas1011@gmail.com \\ * Correspondence: m.akram@pucit.edu.pk
}

Received: 17 November 2019; Accepted: 12 December 2019; Published: 13 December 2019

\begin{abstract}
Graphs play a pivotal role in structuring real-world scenarios such as network security and expert systems. Numerous extensions of graph theoretical conceptions have been established for modeling uncertainty in graphical network situations. The Pythagorean Dombi fuzzy graph (PDFG), a generalization of the fuzzy Dombi graph (FDG), is very useful in representing vague relations between several objects, whereas the operational parameter has a flexible nature in decision-making problems. The main objective of this research study is to expand the area of discussion on PDFGs by establishing fruitful results and notions related to operations such as the direct product, Cartesian product, semi-strong product, strong product, and composition on PDFGs. Certain concepts, including the degree of vertices and total degree, are discussed as its modifications. Meanwhile, these outcomes are considered on PDFGs maintaining the strongness property. At the end, an algorithm for Pythagorean Dombi fuzzy multi-criteria decision-making is given, and a numerical example based on the selection of a leading textile industry is put forward to clarify the suitability of the proposed approach.
\end{abstract}

Keywords: Pythagorean Dombi fuzzy graphs; operations on PDFGs; strongness property; decision-making application

\section{Introduction}

In the last several years, many operators were established that occurred in various monographs with regard to fuzzy logic; specifically, min-max, Frank, Einstein, product, Hamacher, and Dombi operators. These parametric families gain one's attention from a practical point of view as different arguments can be made by taking into account different values of the parameters.

Zadeh [1] proposed the concept of the min operator for introducing a fuzzy set (FS). Hamacher [2] showed that these operators can be easily created by considering the solution of associative operation equality. Later, rational structures were obtained under Kuwagaki's results [3]. From that time, a more generalized form, i.e., triangular norms (t-norms) and triangular conorms ( $\mathrm{t}$-conorms), was explored by the scholars active in the fuzzy theory area. Within the probabilistic metric framework, $t$-norms and $\mathrm{t}$-conorms were initiated by Menger [4], where the distance between objects was narrated by numbers. Many axioms concerning t-norms and t-conorms were given by Schweizer and Sklar [5]. Furthermore, Alsina et al. [6] certified these norms as standard models for defining the union and intersection of FSs. Several summarizations and extensions of meaningful results of $\mathcal{T}$-operators can be observed in Klement et al. [7] and [8], respectively. Zadeh's min and max operators have been widely 
used in almost every fuzzy logic application, but from the theoretical and experimental perspective, other $\mathcal{T}$-operators may produce better outcomes, especially in decision-making situations, such as the product operator may be preferred over the minimum operator [9]. Before the appropriate choice of these operators, one has to examine the features of $\mathcal{T}$-operators like simplicity, suitability, and the implementation of software and hardware. Since the work and study on these operators has expanded, a variety of choices exists for selecting $\mathcal{T}$-operators that may be preferred for a given analysis.

Graphs connect objects, but if in the connection there exists vagueness, then it can be considered as a fuzzy graph (FG). Rosenfeld [10] presented the structure of FG by establishing the fuzzy relation on FS with minimum and maximum operators. Further, several operations on FGs were discussed by Mordeson and Peng [11]. As the hesitant part was not clearly expressed, therefore Atanassov [12] generalized FSs to intuitionistic fuzzy sets (IFSs) by appointing membership $\mu$ and non-membership grade $v$ to the units, fulfilling the requirement $\mu+v \leq 1$ with hesitant part $\pi=1-\mu-v$. Due to the wide range of graph theory applications, Shannon and Atanassov [13] gave the proposal of IFGsby taking into account intuitionistic fuzzy relations on IFSs. To deal with impreciseness and complex uncertainty, Yager [14-16] developed Pythagorean fuzzy sets (PFSs) with the requirement $\mu^{2}+v^{2} \leq 1$, where $v$ and $\mu$ depict non-membership and membership grade, respectively. After that, the dual aspects of a unit were explained by Zhang and Xu [17] with the Pythagorean fuzzy number (PFN). In any decision-making atmosphere, the encouragement of PFSs can be seen; a professional provides the preferable information with membership $\mu=0.8$ and non-membership $v=0.3$, and one may observe that the IF number fails to tackle this state, as $0.8+0.3>1$. Besides, $(0.8)^{2}+(0.3)^{2} \leq 1$. Hence, a greater amount of haziness can be dealt with by the help of PFSs. The concept of PFS has been prosperously applied in numerous fields [17,18]. In practical MCGDM situations, Akram et al. [19,20] proved that PFSs are much more reliable in handling haziness. Under the PF environment, some operations [21] and the TODIM technique for MCDM problem [22] have been studied. Moreover, it has been examined from several perspectives, in particular aggregation operators [23,24]. Garg [25-28] inspected and explored numerous applications of PFSs in a decision support system. As an extensive range of applications, such as database theory, optimization of networks, and decision-making are covered by means of graphs, hence on this basis, Naz et al. [29] presented the notion of Pythagorean fuzzy graphs (PFGs) by considering min and max operators. Verma et al. [30] discussed strong PFGs and proffered complements. The energy of PFGs was put forward by Akram and Naz [31]. Under PF circumstances, Akram et al. [32-35] presented certain graphs and analyzed their essential characteristics. Naz et al. [36] developed operations and their application under single valued neutrosophic situation. Akram and Habib [37] discussed the regularity of $q$-rung picture fuzzy graphs with applications. Habib et al. [38] presented the notion of $q$-rung orthopair fuzzy competition graphs by considering the most wide spread max and min operators and gave an application in the soil ecosystem. Akram et al. [39] explored the concept of the edge regularity of $q$-rung picture fuzzy graphs.

Dombi [40] inaugurated the Dombi operator with operational parameter $\lambda$ in 1982. Afterward, he [41] generalized them. The sign of this operational parameter makes it exceptional. It is very helpful in making decisions, as by choosing different values of $\lambda$, distinct arguments can be formed depending on one's requirement. For this precedence, Dombi operations were later used by Chen and Ye [42], Shi and Ye [43], and Jana et al. [44] in MCDM situations. By using the Dombi-Bonferroni mean operator, Liu et al. [45] solved the MCGDM problem. In a hesitant fuzzy environment, He [46] explored typhoon disaster assessment by taking into account Dombi operators. Recently, Akram et al. [47] proffered Pythagorean Dombi fuzzy aggregation operators and gave an application for better understanding. From the existing research, it is noted that in decision-making areas, Dombi operational parameters have an excellent nature. Since FG can easily model and structure decision-making situations with vagueness, a very insufficient attempt has been made to utilize the Dombi operator in graph theory. Hence, on this base, Ashraf et al. [48] provided the notion of the Dombi fuzzy graph (DFG). As PF models are more versatile and practical than fuzzy and IF models for describing uncertain information that appear in decision-making circumstances, such as mathematics, engineering, medical, artificial 
intelligence, and social sciences, Akram et al. [49] presented the idea of PDFG by considering the point that for extending classical graphs to PFGs, max and min operators are not always preferred to deal with certain world problems. Furthermore, the development inaugurated by Klement, Hamachar, Alsina, and other inventors was put together in the area of PFG theory, and the use of the $\mathcal{T}$-operator, mainly, the Dombi operator was demonstrated. Graph operations produce new classes of graphs from initial ones, which in turn may be useful for the modeling and recognition of computer network designs. In this research article, various operations of the proposed graph, such as the direct product, semi-strong product, strong product, Cartesian product, and composition, are developed, and a number of their significant characteristics are explored, as they are widely used for structuring reliable models. These graph products can be utilized to create and examine series of real-world networks, in particular, communication and road networks. Further, the degree of vertices and total degree are defined as a modification, of the resultant PDFGs, acquired from the given PDFGs using these operations.

The presented research article is structured as follows: Section 2 proposes the novel concept of certain Pythagorean Dombi fuzzy graphs such as direct, Cartesian, semi-strong, strong, and composition with appropriate illustrations. Section 3 presents a decision-making algorithm in the Pythagorean Dombi fuzzy environment and solves a numerical example to illustrate the developed method. Section 4 contains concluding remarks and points out directions for future work.

\section{Certain Pythagorean Dombi Fuzzy Graphs}

In this section, certain Pythagorean Dombi fuzzy graphs including direct, Cartesian, semi-strong, strong, and composition are defined with their essential features, as these graphs play a crucial role in structuring and designing reliable communication and road networking models.

\subsection{Direct Product of Pythagorean Dombi Fuzzy Graphs}

Definition 1. Let $\mathbf{A}_{j}$ and $\mathbf{B}_{j}$ be the Pythagorean fuzzy subsets of $\mathbf{V}_{j}$ and $\mathbf{E}_{j}(j=1,2)$, respectively. The direct product of PDFGs $\mathbf{G}_{1}=\left(\mathbf{A}_{1}, \mathbf{B}_{1}\right)$ and $\mathbf{G}_{2}=\left(\mathbf{A}_{2}, \mathbf{B}_{2}\right)$ of underlying crisp graphs $\mathbf{G}_{1}^{\prime}=\left(\mathbf{V}_{1}, \mathbf{E}_{1}\right)$ and $\mathbf{G}_{2}^{\prime}=\left(\mathbf{V}_{2}, \mathbf{E}_{2}\right)$, respectively, is represented by $\mathbf{G}_{1} \times \mathbf{G}_{2}=\left(\mathbf{A}_{1} \times \mathbf{A}_{2}, \mathbf{B}_{1} \times \mathbf{B}_{2}\right)$, defined as:

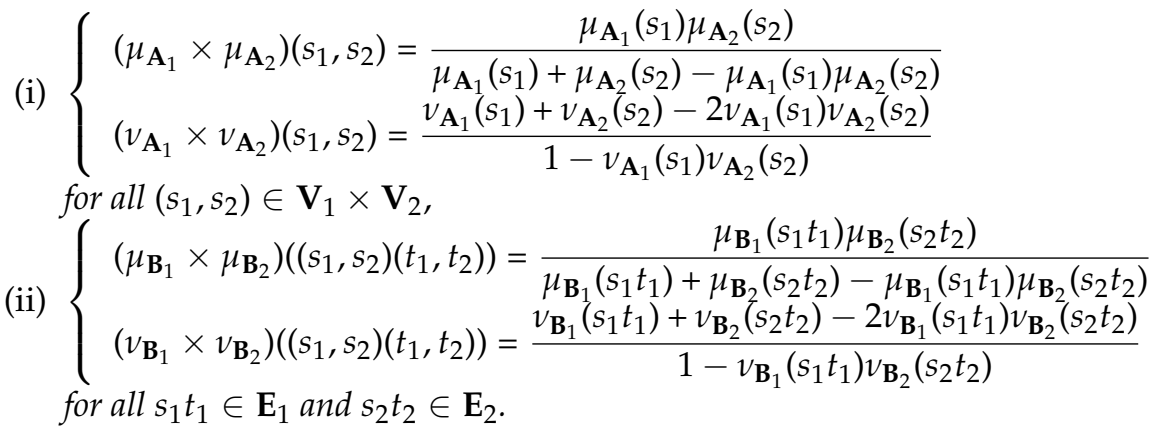

Example 1. Consider PDFGs $\mathbf{G}_{1}=\left(\mathbf{A}_{1}, \mathbf{B}_{1}\right)$ and $\mathbf{G}_{2}=\left(\mathbf{A}_{2}, \mathbf{B}_{2}\right)$ on $\mathbf{V}_{1}=\left\{s_{1}, s_{2}\right\}$ and $\mathbf{V}_{2}=\left\{t_{1}, t_{2}\right\}$, respectively, as represented in Figure 1. Their direct product $\mathbf{G}_{1} \times \mathbf{G}_{2}$ is given in Figure 2 .

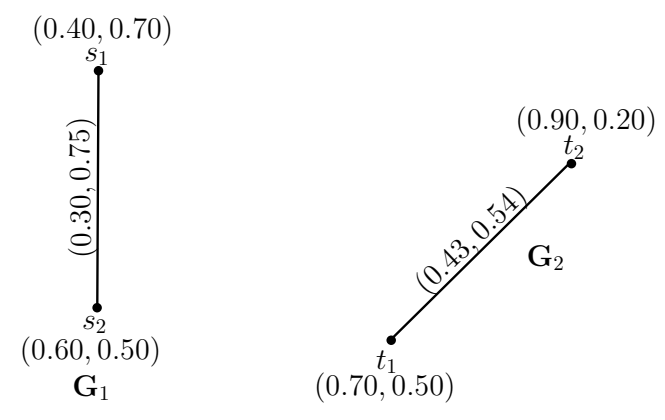

Figure 1. Pythagorean Dombi fuzzy graphs. 


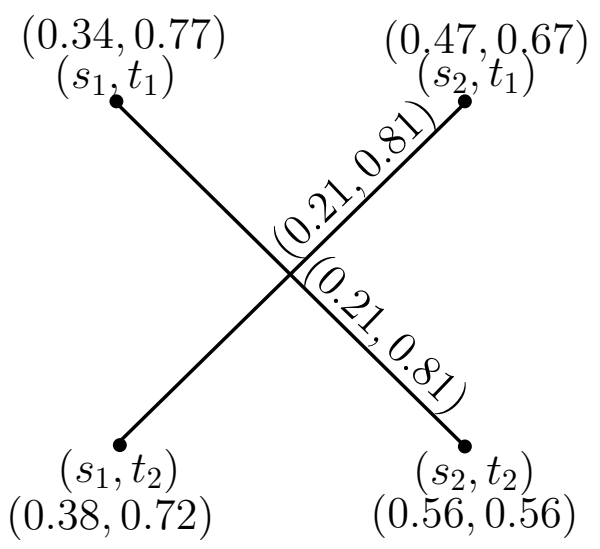

Figure 2. Direct product of two PDFGs.

By routine computations, one can view from Figure 2 that $\mathbf{G}_{1} \times \mathbf{G}_{2}=\left(\mathbf{A}_{1} \times \mathbf{A}_{2}, \mathbf{B}_{1} \times \mathbf{B}_{2}\right)$ is a PDFG of the underlying crisp graph $\mathbf{G}_{1}^{\prime} \times \mathbf{G}_{2}^{\prime}=\left(\mathbf{V}_{1} \times \mathbf{V}_{2}, \mathbf{E}_{1} \times \mathbf{E}_{2}\right)$.

Proposition 1. If $\mathbf{G}_{1}=\left(\mathbf{A}_{1}, \mathbf{B}_{1}\right)$ and $\mathbf{G}_{2}=\left(\mathbf{A}_{2}, \mathbf{B}_{2}\right)$ are two PDFGs of underlying crisp graphs $\mathbf{G}_{1}^{\prime}=\left(\mathbf{V}_{1}, \mathbf{E}_{1}\right)$ and $\mathbf{G}_{2}^{\prime}=\left(\mathbf{V}_{2}, \mathbf{E}_{2}\right)$, respectively, then the direct product $\mathbf{G}_{1} \times \mathbf{G}_{2}$ of $\mathbf{G}_{1}$ and $\mathbf{G}_{2}$ is the PDFG of $\mathbf{G}_{1}^{\prime} \times \mathbf{G}_{2}^{\prime}$.

Proof. Assume that $\mathrm{G}_{1}$ and $\mathrm{G}_{2}$ are two PDFGs of underlying crisp graphs $\mathbf{G}_{1}^{\prime}$ and $\mathrm{G}_{2}^{\prime}$, respectively. Further, suppose that $\mathbf{G}=\mathbf{G}_{1} \times \mathbf{G}_{2}=\left(\mathbf{A}_{1} \times \mathbf{A}_{2}, \mathbf{B}_{1} \times \mathbf{B}_{2}\right)$ is the direct product of $\mathbf{G}_{1}$ and $\mathbf{G}_{2}$, where $\mathbf{A}=\mathbf{A}_{1} \times \mathbf{A}_{2}$ and $\mathbf{B}=\mathbf{B}_{1} \times \mathbf{B}_{2}$ are the PF vertex and edge set, respectively. Let $\left(s_{1}, s_{2}\right)\left(t_{1}, t_{2}\right) \in \mathbf{E}_{1} \times \mathbf{E}_{2}$.

If $s_{1} t_{1} \in \mathbf{E}_{1}$ and $s_{2} t_{2} \in \mathbf{E}_{2}$, then the membership grade is:

$$
\begin{aligned}
\left(\mu_{\mathbf{B}_{1}} \times \mu_{\mathbf{B}_{2}}\right)\left(\left(s_{1}, s_{2}\right)\left(t_{1}, t_{2}\right)\right) & =\mathcal{T}\left(\mu_{\mathbf{B}_{1}}\left(s_{1} t_{1}\right), \mu_{\mathbf{B}_{2}}\left(s_{2} t_{2}\right)\right) \\
& \leq \mathcal{T}\left(\frac{\mu_{\mathbf{A}_{1}}\left(s_{1}\right) \mu_{\mathbf{A}_{1}}\left(t_{1}\right)}{\mu_{\mathbf{A}_{1}}\left(s_{1}\right)+\mu_{\mathbf{A}_{1}}\left(t_{1}\right)-\mu_{\mathbf{A}_{1}}\left(s_{1}\right) \mu_{\mathbf{A}_{1}}\left(t_{1}\right)},\right. \\
& \left.\frac{\mu_{\mathbf{A}_{2}}\left(s_{2}\right) \mu_{\mathbf{A}_{2}}\left(t_{2}\right)}{\mu_{\mathbf{A}_{2}}\left(s_{2}\right)+\mu_{\mathbf{A}_{2}}\left(t_{2}\right)-\mu_{\mathbf{A}_{2}}\left(s_{2}\right) \mu_{\mathbf{A}_{2}}\left(t_{2}\right)}\right)
\end{aligned}
$$

Taking $\mu_{\mathbf{A}_{1}}\left(s_{1}\right)=u, \mu_{\mathbf{A}_{1}}\left(t_{1}\right)=v, \mu_{\mathbf{A}_{2}}\left(s_{2}\right)=w$, and $\mu_{\mathbf{A}_{2}}\left(t_{2}\right)=x$, we have:

$$
\begin{aligned}
& \left(\mu_{\mathbf{B}_{1}} \times \mu_{\mathbf{B}_{2}}\right)\left(\left(s_{1}, s_{2}\right)\left(t_{1}, t_{2}\right)\right) \leq \mathcal{T}\left(\frac{u v}{u+v-u v}, \frac{w x}{w+x-w x}\right) \\
& =\frac{\frac{u v w x}{(u+v-u v)(w+x-w x)}}{\frac{u v}{u+v-u v}+\frac{w x}{w+x-w x}-\frac{u v w x}{(u+v-u v)(w+x-w x)}} \\
& =\frac{\frac{u v w x}{(u+w-u w)(v+x-v x)}}{\frac{u v}{u+w-u w}+\frac{w x}{v+x-v x}-\frac{u v w x}{(u+w-u w)(v+x-v x)}} \\
& =\frac{\left(\mu_{\mathbf{A}_{1}} \times \mu_{\mathbf{A}_{2}}\right)\left(s_{1}, s_{2}\right)\left(\mu_{\mathbf{A}_{1}} \times \mu_{\mathbf{A}_{2}}\right)\left(t_{1}, t_{2}\right)}{\left(\mu_{\mathbf{A}_{1}} \times \mu_{\mathbf{A}_{2}}\right)\left(s_{1}, s_{2}\right)+\left(\mu_{\mathbf{A}_{1}} \times \mu_{\mathbf{A}_{2}}\right)\left(t_{1}, t_{2}\right)-\left(\mu_{\mathbf{A}_{1}} \times \mu_{\mathbf{A}_{2}}\right)\left(s_{1}, s_{2}\right)} . \\
& \left(\mu_{\mathbf{A}_{1}} \times \mu_{\mathbf{A}_{2}}\right)\left(t_{1}, t_{2}\right)
\end{aligned}
$$


Likewise, for the non-membership grade:

$$
\begin{aligned}
\left(v_{\mathbf{B}_{1}} \times v_{\mathbf{B}_{2}}\right)\left(\left(s_{1}, s_{2}\right)\left(t_{1}, t_{2}\right)\right) & =\mathcal{S}\left(v_{\mathbf{B}_{1}}\left(s_{1} t_{1}\right), v_{\mathbf{B}_{2}}\left(s_{2} t_{2}\right)\right) \\
& \leq \mathcal{S}\left(\frac{v_{\mathbf{A}_{1}}\left(s_{1}\right)+v_{\mathbf{A}_{1}}\left(t_{1}\right)-2 v_{\mathbf{A}_{1}}\left(s_{1}\right) v_{\mathbf{A}_{1}}\left(t_{1}\right)}{1-v_{\mathbf{A}_{1}}\left(s_{1}\right) v_{\mathbf{A}_{1}}\left(t_{1}\right)},\right. \\
& \left.\frac{v_{\mathbf{A}_{2}}\left(s_{2}\right)+v_{\mathbf{A}_{2}}\left(t_{2}\right)-2 v_{\mathbf{A}_{2}}\left(s_{2}\right) v_{\mathbf{A}_{2}}\left(t_{2}\right)}{1-v_{\mathbf{A}_{2}}\left(s_{2}\right) v_{\mathbf{A}_{2}}\left(t_{2}\right)}\right)
\end{aligned}
$$

Taking $v_{\mathbf{A}_{1}}\left(s_{1}\right)=p, v_{\mathbf{A}_{1}}\left(t_{1}\right)=q, v_{\mathbf{A}_{2}}\left(s_{2}\right)=n$ and $v_{\mathbf{A}_{2}}\left(t_{2}\right)=m$, we have:

$$
\begin{aligned}
& \left(v_{\mathbf{B}_{1}} \times v_{\mathbf{B}_{2}}\right)\left(\left(s_{1}, s_{2}\right)\left(t_{1}, t_{2}\right)\right) \leq \mathcal{S}\left(\frac{p+q-2 p q}{1-p q}, \frac{n+m-2 n m}{1-n m}\right) \\
& =\frac{\frac{p+q-2 p q}{1-p q}+\frac{n+m-2 n m}{1-n m}-2\left(\frac{p+q-2 p q}{1-p q}\right)\left(\frac{n+m-2 n m}{1-n m}\right)}{1-\left(\frac{p+q-2 p q}{1-p q}\right)\left(\frac{n+m-2 n m}{1-n m}\right)} \\
& =\frac{\frac{p+n-2 p n}{1-p n}+\frac{q+m-2 q m}{1-q m}-2\left(\frac{p+n-2 p n}{1-p n}\right)\left(\frac{q+m-2 q m}{1-q m}\right)}{1-\left(\frac{p+n-2 p n}{1-p n}\right)\left(\frac{q+m-2 q m}{1-q m}\right)} \\
& \left(v_{\mathbf{A}_{1}} \times v_{\mathbf{A}_{2}}\right)\left(s_{1}, s_{2}\right)+\left(v_{\mathbf{A}_{1}} \times v_{\mathbf{A}_{2}}\right)\left(t_{1}, t_{2}\right)-2\left(v_{\mathbf{A}_{1}} \times v_{\mathbf{A}_{2}}\right)\left(s_{1}, s_{2}\right) \\
& =\frac{\left(v_{\mathbf{A}_{1}} \times v_{\mathbf{A}_{2}}\right)\left(t_{1}, t_{2}\right)}{1-\left(v_{\mathbf{A}_{1}} \times v_{\mathbf{A}_{2}}\right)\left(s_{1}, s_{2}\right)\left(v_{\mathbf{A}_{1}} \times v_{\mathbf{A}_{2}}\right)\left(t_{1}, t_{2}\right)} .
\end{aligned}
$$

Hence, it is concluded that $\mathbf{G}_{1} \times \mathrm{G}_{2}$ is a PDFG of $\mathbf{G}_{1}^{\prime} \times \mathrm{G}_{2}^{\prime}$.

Definition 2. Consider $\mathbf{G}_{1}=\left(\mathbf{A}_{1}, \mathbf{B}_{1}\right)$ and $\mathbf{G}_{2}=\left(\mathbf{A}_{2}, \mathbf{B}_{2}\right)$ to be two PDFGs. Then, for any vertex $\left(s_{1}, s_{2}\right) \in$ $\mathbf{V}_{1} \times \mathbf{V}_{2}$

$$
\begin{aligned}
\left(\mathbf{D}_{\mu}\right)_{\mathbf{G}_{1} \times \mathbf{G}_{2}}\left(s_{1}, s_{2}\right) & =\sum_{\left(s_{1}, s_{2}\right)\left(t_{1}, t_{2}\right) \in \mathbf{E}_{1} \times \mathbf{E}_{2}}\left(\mu_{\mathbf{B}_{1}} \times \mu_{\mathbf{B}_{2}}\right)\left(\left(s_{1}, s_{2}\right)\left(t_{1}, t_{2}\right)\right) \\
& =\sum_{s_{1} t_{1} \in \mathbf{E}_{1}, s_{2} t_{2} \in \mathbf{E}_{2}} \frac{\mu_{\mathbf{B}_{1}}\left(s_{1} t_{1}\right) \mu_{\mathbf{B}_{2}}\left(s_{2} t_{2}\right)}{\mu_{\mathbf{B}_{1}}\left(s_{1} t_{1}\right)+\mu_{\mathbf{B}_{2}}\left(s_{2} t_{2}\right)-\mu_{\mathbf{B}_{1}}\left(s_{1} t_{1}\right) \mu_{\mathbf{B}_{2}}\left(s_{2} t_{2}\right)}, \\
\left(\mathbf{D}_{v}\right)_{\mathbf{G}_{1} \times \mathbf{G}_{2}}\left(s_{1}, s_{2}\right) & =\sum_{\left(s_{1}, s_{2}\right)\left(t_{1}, t_{2}\right) \in \mathbf{E}_{1} \times \mathbf{E}_{2}}\left(v_{\mathbf{B}_{1}} \times v_{\mathbf{B}_{2}}\right)\left(\left(s_{1}, s_{2}\right)\left(t_{1}, t_{2}\right)\right) \\
& =\sum_{s_{1} t_{1} \in \mathbf{E}_{1}, s_{2} t_{2} \in \mathbf{E}_{2}} \frac{v_{\mathbf{B}_{1}}\left(s_{1} t_{1}\right)+v_{\mathbf{B}_{2}}\left(s_{2} t_{2}\right)-2 v_{\mathbf{B}_{1}}\left(s_{1} t_{1}\right) v_{\mathbf{B}_{2}}\left(s_{2} t_{2}\right)}{1-v_{\mathbf{B}_{1}}\left(s_{1} t_{1}\right) v_{\mathbf{B}_{2}}\left(s_{2} t_{2}\right)} .
\end{aligned}
$$

Definition 3. Consider $\mathbf{G}_{1}=\left(\mathbf{A}_{1}, \mathbf{B}_{1}\right)$ and $\mathbf{G}_{2}=\left(\mathbf{A}_{2}, \mathbf{B}_{2}\right)$ to be two PDFGs. Then, for any vertex $\left(s_{1}, s_{2}\right) \in$ $\mathbf{V}_{1} \times \mathbf{V}_{2}$

$$
\begin{aligned}
\left(\mathbf{T D}_{\mu}\right)_{\mathbf{G}_{1} \times \mathbf{G}_{2}}\left(s_{1}, s_{2}\right) & =\sum_{\left(s_{1}, s_{2}\right)\left(t_{1}, t_{2}\right) \in \mathbf{E}_{1} \times \mathbf{E}_{2}}\left(\mu_{\mathbf{B}_{1}} \times \mu_{\mathbf{B}_{2}}\right)\left(\left(s_{1}, s_{2}\right)\left(t_{1}, t_{2}\right)\right)+\left(\mu_{\mathbf{A}_{1}} \times \mu_{\mathbf{A}_{2}}\right)\left(s_{1}, s_{2}\right) \\
& =\sum_{s_{1} t_{1} \in \mathbf{E}_{1}, s_{2} t_{2} \in \mathbf{E}_{2}} \frac{\mu_{\mathbf{B}_{1}}\left(s_{1} t_{1}\right) \mu_{\mathbf{B}_{2}}\left(s_{2} t_{2}\right)}{\mu_{\mathbf{B}_{1}}\left(s_{1} t_{1}\right)+\mu_{\mathbf{B}_{2}}\left(s_{2} t_{2}\right)-\mu_{\mathbf{B}_{1}}\left(s_{1} t_{1}\right) \mu_{\mathbf{B}_{2}}\left(s_{2} t_{2}\right)} \\
& +\frac{\mu_{\mathbf{A}_{1}}\left(s_{1}\right) \mu_{\mathbf{A}_{2}}\left(s_{2}\right)}{\mu_{\mathbf{A}_{1}}\left(s_{1}\right)+\mu_{\mathbf{A}_{2}}\left(s_{2}\right)-\mu_{\mathbf{A}_{1}}\left(s_{1}\right) \mu_{\mathbf{A}_{2}}\left(s_{2}\right)}
\end{aligned}
$$




$$
\begin{aligned}
\left(\mathbf{T D}_{v}\right)_{\mathbf{G}_{1} \times \mathbf{G}_{2}}\left(s_{1}, s_{2}\right) & =\sum_{\left(s_{1}, s_{2}\right)\left(t_{1}, t_{2}\right) \in \mathbf{E}_{1} \times \mathbf{E}_{2}}\left(v_{\mathbf{B}_{1}} \times v_{\mathbf{B}_{2}}\right)\left(\left(s_{1}, s_{2}\right)\left(t_{1}, t_{2}\right)\right)+\left(v_{\mathbf{A}_{1}} \times v_{\mathbf{A}_{2}}\right)\left(s_{1}, s_{2}\right) \\
& =\sum_{s_{1} t_{1} \in \mathbf{E}_{1}, s_{2} t_{2} \in \mathbf{E}_{2}} \frac{v_{\mathbf{B}_{1}}\left(s_{1} t_{1}\right)+v_{\mathbf{B}_{2}}\left(s_{2} t_{2}\right)-2 v_{\mathbf{B}_{1}}\left(s_{1} t_{1}\right) v_{\mathbf{B}_{2}}\left(s_{2} t_{2}\right)}{1-v_{\mathbf{B}_{1}}\left(s_{1} t_{1}\right) v_{\mathbf{B}_{2}}\left(s_{2} t_{2}\right)} \\
& +\frac{v_{\mathbf{A}_{1}}\left(s_{1}\right)+v_{\mathbf{A}_{2}}\left(s_{2}\right)-2 v_{\mathbf{A}_{1}}\left(s_{1}\right) v_{\mathbf{A}_{2}}\left(s_{2}\right)}{1-v_{\mathbf{A}_{1}}\left(s_{1}\right) v_{\mathbf{A}_{2}}\left(s_{2}\right)}
\end{aligned}
$$

Example 2. Consider PDFGs $\mathbf{G}_{1}$ and $\mathbf{G}_{2}$ as in Example 1. Their direct product is presented in Figure 2. Then, by Definition 2, we must have:

$$
\begin{aligned}
& \left(\mathbf{D}_{\mu}\right)_{\mathbf{G}_{1} \times \mathbf{G}_{2}}\left(s_{1}, t_{2}\right)=\left(\mu_{\mathbf{B}_{1}} \times \mu_{\mathbf{B}_{2}}\right)\left(\left(s_{1}, t_{2}\right)\left(s_{2}, t_{1}\right)\right)=\frac{\mu_{\mathbf{B}_{1}}\left(s_{1} s_{2}\right) \mu_{\mathbf{B}_{2}}\left(t_{1} t_{2}\right)}{\mu_{\mathbf{B}_{1}}\left(s_{1} s_{2}\right)+\mu_{\mathbf{B}_{2}}\left(t_{1} t_{2}\right)-\mu_{\mathbf{B}_{1}}\left(s_{1} s_{2}\right) \mu_{\mathbf{B}_{2}}\left(t_{1} t_{2}\right)}=0.21, \\
& \left(\mathbf{D}_{v}\right)_{\mathbf{G}_{1} \times \mathbf{G}_{2}}\left(s_{1}, t_{2}\right)=\left(v_{\mathbf{B}_{1}} \times v_{\mathbf{B}_{2}}\right)\left(\left(s_{1}, t_{2}\right)\left(s_{2}, t_{1}\right)\right)=\frac{v_{\mathbf{B}_{1}}\left(s_{1} s_{2}\right)+v_{\mathbf{B}_{2}}\left(t_{1} t_{2}\right)-2 v_{\mathbf{B}_{1}}\left(s_{1} s_{2}\right) \nu_{\mathbf{B}_{2}}\left(t_{1} t_{2}\right)}{1-v_{\mathbf{B}_{1}}\left(s_{1} s_{2}\right) v_{\mathbf{B}_{2}}\left(t_{1} t_{2}\right)}=0.81 .
\end{aligned}
$$

Thus, $(\mathbf{D})_{\mathbf{G}_{1} \times \mathbf{G}_{2}}\left(s_{1}, t_{2}\right)=(0.21,0.81)$.

Further, by using Definition 3, we have:

$$
\begin{aligned}
\left(\mathbf{T D}_{\mu}\right)_{\mathbf{G}_{1} \times \mathbf{G}_{2}}\left(s_{1}, t_{2}\right) & =\left(\mathbf{D}_{\mu}\right)_{\mathbf{G}_{1} \times \mathbf{G}_{2}}\left(s_{1}, t_{2}\right)+\left(\mu_{\mathbf{A}_{1}} \times \mu_{\mathbf{A}_{2}}\right)\left(s_{1}, t_{2}\right) \\
& =0.21+\frac{\mu_{\mathbf{A}_{1}}\left(s_{1}\right) \mu_{\mathbf{A}_{2}}\left(t_{2}\right)}{\mu_{\mathbf{A}_{1}}\left(s_{1}\right)+\mu_{\mathbf{A}_{2}}\left(t_{2}\right)-\mu_{\mathbf{A}_{1}}\left(s_{1}\right) \mu_{\mathbf{A}_{2}}\left(t_{2}\right)}=0.59, \\
\left(\mathbf{T D}_{v}\right)_{\mathbf{G}_{1} \times \mathbf{G}_{2}}\left(s_{1}, t_{2}\right) & =\left(\mathbf{D}_{v}\right)_{\mathbf{G}_{1} \times \mathbf{G}_{2}}\left(s_{1}, t_{2}\right)+\left(v_{\mathbf{A}_{1}} \times v_{\mathbf{A}_{2}}\right)\left(s_{1}, t_{2}\right) \\
& =0.81+\frac{v_{\mathbf{A}_{1}}\left(s_{1}\right)+v_{\mathbf{A}_{2}}\left(t_{2}\right)-2 v_{\mathbf{A}_{1}}\left(s_{1}\right) v_{\mathbf{A}_{2}}\left(t_{2}\right)}{1-v_{\mathbf{A}_{1}}\left(s_{1}\right) v_{\mathbf{A}_{2}}\left(t_{2}\right)}=1.53 .
\end{aligned}
$$

Thus, $(\mathbf{T D})_{\mathbf{G}_{1} \times \mathbf{G}_{2}}\left(s_{1}, t_{2}\right)=(0.59,1.53)$.

Proposition 2. If $\mathbf{G}_{1}=\left(\mathbf{A}_{1}, \mathbf{B}_{1}\right)$ and $\mathbf{G}_{2}=\left(\mathbf{A}_{2}, \mathbf{B}_{2}\right)$ are strong PDFGs of underlying crisp graphs $\mathbf{G}_{1}^{\prime}=$ $\left(\mathbf{V}_{1}, \mathbf{E}_{1}\right)$ and $\mathbf{G}_{2}^{\prime}=\left(\mathbf{V}_{2}, \mathbf{E}_{2}\right)$, respectively, then direct product $\mathbf{G}_{1} \times \mathbf{G}_{2}$ of $\mathbf{G}_{1}$ and $\mathbf{G}_{2}$ is also a strong PDFG of $\mathbf{G}_{1}^{\prime} \times \mathbf{G}_{2}^{\prime}$.

Proof. The proof is the same as Proposition 1.

Proposition 3. If $\mathbf{G}_{1} \times \mathbf{G}_{2}$ of $\mathbf{G}_{1}$ and $\mathbf{G}_{2}$ is a strong PDFG, then at least $\mathbf{G}_{1}$ or $\mathbf{G}_{2}$ must be a strong PDFG.

Proof. Suppose on the contrary that $\mathbf{G}_{1}$ and $\mathbf{G}_{2}$ are not strong PDFGs. Then, for $s_{1} t_{1} \in E_{1}$ and $s_{2} t_{2} \in E_{2}$, we have:

$$
\begin{gathered}
\mu_{\mathbf{B}_{1}}\left(s_{1} t_{1}\right)<\frac{\mu_{\mathbf{A}_{1}}\left(s_{1}\right) \mu_{\mathbf{A}_{1}}\left(t_{1}\right)}{\mu_{\mathbf{A}_{1}}\left(s_{1}\right)+\mu_{\mathbf{A}_{1}}\left(t_{1}\right)-\mu_{\mathbf{A}_{1}}\left(s_{1}\right) \mu_{\mathbf{A}_{1}}\left(t_{1}\right)}=\frac{u v}{u+v-u v}, \\
v_{\mathbf{B}_{1}}\left(s_{1} t_{1}\right)<\frac{v_{\mathbf{A}_{1}}\left(s_{1}\right)+v_{\mathbf{A}_{1}}\left(t_{1}\right)-2 v_{\mathbf{A}_{1}}\left(s_{1}\right) \nu_{\mathbf{A}_{1}}\left(t_{1}\right)}{1-v_{\mathbf{A}_{1}}\left(s_{1}\right) v_{\mathbf{A}_{2}}\left(t_{1}\right)}=\frac{p+q-2 p q}{1-p q},
\end{gathered}
$$

and:

$$
\begin{gathered}
\mu_{\mathbf{B}_{2}}\left(s_{2} t_{2}\right)<\frac{\mu_{\mathbf{A}_{2}}\left(s_{2}\right) \mu_{\mathbf{A}_{2}}\left(t_{2}\right)}{\mu_{\mathbf{A}_{2}}\left(s_{2}\right)+\mu_{\mathbf{A}_{2}}\left(t_{2}\right)-\mu_{\mathbf{A}_{2}}\left(s_{2}\right) \mu_{\mathbf{A}_{2}}\left(t_{2}\right)}=\frac{w x}{w+x-w x}, \\
v_{\mathbf{B}_{2}}\left(s_{2} t_{2}\right)<\frac{v_{\mathbf{A}_{2}}\left(s_{2}\right)+v_{\mathbf{A}_{2}}\left(t_{2}\right)-2 v_{\mathbf{A}_{2}}\left(s_{2}\right) v_{\mathbf{A}_{2}}\left(t_{2}\right)}{1-v_{\mathbf{A}_{2}}\left(s_{2}\right) \nu_{\mathbf{A}_{2}}\left(t_{2}\right)}=\frac{n+m-2 n m}{1-n m} .
\end{gathered}
$$


Assume that:

$$
\begin{gathered}
\mu_{\mathbf{B}_{2}}\left(s_{2} t_{2}\right) \leq \mu_{\mathbf{B}_{1}}\left(s_{1} t_{1}\right)<\frac{u v}{u+v-u v} \leq u, \\
v_{\mathbf{B}_{2}}\left(s_{2} t_{2}\right) \leq v_{\mathbf{B}_{1}}\left(s_{1} t_{1}\right)<\frac{p+q-2 p q}{1-p g} \leq p+q-p q .
\end{gathered}
$$

Let $\left(s_{1}, s_{2}\right)\left(t_{1}, t_{2}\right) \in E_{1} \times E_{2}$. If $s_{1} t_{1} \in E_{1}$ and $s_{2} t_{2} \in E_{2}$, then the membership grade is:

$$
\begin{aligned}
& \left(\mu_{\mathbf{B}_{1}} \times \mu_{\mathbf{B}_{2}}\right)\left(\left(s_{1}, s_{2}\right)\left(t_{1}, t_{2}\right)\right)=\mathcal{T}\left(\mu_{\mathbf{B}_{1}}\left(s_{1} t_{1}\right), \mu_{\mathbf{B}_{2}}\left(s_{2} t_{2}\right)\right) \\
& <\mathcal{T}\left(\frac{u v}{u+v-u v}, \frac{w x}{w+x-w x}\right) \\
& =\frac{\frac{u v w x}{(u+v-u v)(w+x-w x)}}{\frac{u v w x}{u+v-u v}+\frac{w x}{w+x-w x}-\frac{u v w)(w+x-w x)}{(u+v-u v)(w+x)}} \\
& =\frac{\overline{(u+w-u w)(v+x-v x)}}{\frac{u v}{u+w-u w}+\frac{w x}{v+x-v x}-\frac{u v w}{(u+w-u w)(v+x-v x)}} \\
& =\frac{\left(\mu_{\mathbf{A}_{1}} \times \mu_{\mathbf{A}_{2}}\right)\left(s_{1}, s_{2}\right)\left(\mu_{\mathbf{A}_{1}} \times \mu_{\mathbf{A}_{2}}\right)\left(t_{1}, t_{2}\right)}{\left(\mu_{\mathbf{A}_{1}} \times \mu_{\mathbf{A}_{2}}\right)\left(s_{1}, s_{2}\right)+\left(\mu_{\mathbf{A}_{1}} \times \mu_{\mathbf{A}_{2}}\right)\left(t_{1}, t_{2}\right)-\left(\mu_{\mathbf{A}_{1}} \times \mu_{\mathbf{A}_{2}}\right)\left(s_{1}, s_{2}\right)} . \\
& \left(\mu_{\mathbf{A}_{1}} \times \mu_{\mathbf{A}_{2}}\right)\left(t_{1}, t_{2}\right)
\end{aligned}
$$

Likewise, for the non-membership grade:

$$
\begin{aligned}
& \left(v_{\mathbf{B}_{1}} \times v_{\mathbf{B}_{2}}\right)\left(\left(s_{1}, s_{2}\right)\left(t_{1}, t_{2}\right)\right)=\mathcal{S}\left(v_{\mathbf{B}_{1}}\left(s_{1} t_{1}\right), v_{\mathbf{B}_{2}}\left(s_{2} t_{2}\right)\right) \\
& <\mathcal{S}\left(\frac{p+q-2 p q}{1-p q}, \frac{n+m-2 n m}{1-n m}\right) \\
& =\frac{\frac{p+q-2 p q}{1-p q}+\frac{n+m-2 n m}{1-n m}-2\left(\frac{p+q-2 p q}{1-p q}\right)\left(\frac{n+m-2 n m}{1-n m}\right)}{1-\left(\frac{p+q-2 p q}{1-p q}\right)\left(\frac{n+m-2 n m}{1-n m}\right)} \\
& =\frac{\frac{p+n-2 p n}{1-p n}+\frac{q+m-2 q m}{1-q m}-2\left(\frac{p+n-2 p n}{1-p n}\right)\left(\frac{q+m-2 q m}{1-q m}\right)}{1-\left(\frac{p+n-2 p n}{1-p n}\right)\left(\frac{q+m-2 q m}{1-q m}\right)} \\
& \left(v_{\mathbf{A}_{1}} \times v_{\mathbf{A}_{2}}\right)\left(s_{1}, s_{2}\right)+\left(v_{\mathbf{A}_{1}} \times v_{\mathbf{A}_{2}}\right)\left(t_{1}, t_{2}\right)-2\left(v_{\mathbf{A}_{1}} \times v_{\mathbf{A}_{2}}\right)\left(s_{1}, s_{2}\right) \\
& =\frac{\left(v_{\mathbf{A}_{1}} \times v_{\mathbf{A}_{2}}\right)\left(t_{1}, t_{2}\right)}{1-\left(v_{\mathbf{A}_{1}} \times v_{\mathbf{A}_{2}}\right)\left(s_{1}, s_{2}\right)\left(v_{\mathbf{A}_{1}} \times v_{\mathbf{A}_{2}}\right)\left(t_{1}, t_{2}\right)} .
\end{aligned}
$$

Hence, it is concluded that $\mathbf{G}_{1} \times \mathbf{G}_{2}$ is not a strong PDFG of $\mathbf{G}_{1}^{\prime} \times \mathbf{G}_{2}^{\prime}$, a contradiction.

2.2. Cartesian Product of Pythagorean Dombi Fuzzy Graphs

Definition 4. Let $\mathbf{A}_{j}$ and $\mathbf{B}_{j}$ be the Pythagorean fuzzy subsets of $\mathbf{V}_{j}$ and $\mathbf{E}_{j}(j=1,2)$, respectively. The Cartesian product of PDFGs $\mathbf{G}_{1}=\left(\mathbf{A}_{1}, \mathbf{B}_{1}\right)$ and $\mathbf{G}_{2}=\left(\mathbf{A}_{2}, \mathbf{B}_{2}\right)$ of the underlying crisp graphs $\mathbf{G}_{1}^{\prime}=\left(\mathbf{V}_{1}, \mathbf{E}_{1}\right)$ and $\mathbf{G}_{2}^{\prime}=\left(\mathbf{V}_{2}, \mathbf{E}_{2}\right)$, respectively, is represented by $\mathbf{G}_{1} \square \mathbf{G}_{2}=\left(\mathbf{A}_{1} \square \mathbf{A}_{2}, \mathbf{B}_{1} \square \mathbf{B}_{2}\right)$, defined as:

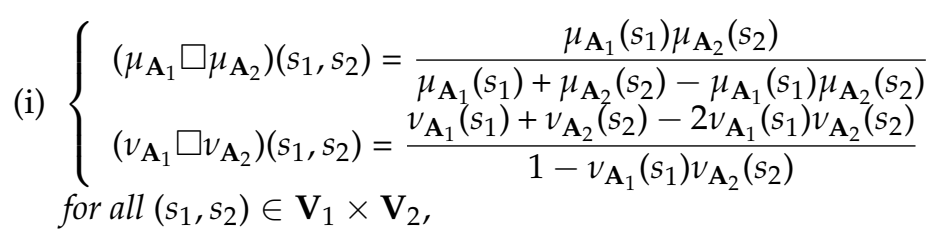


(ii) $\left\{\begin{array}{l}\left(\mu_{\mathbf{B}_{1}} \square \mu_{\mathbf{B}_{2}}\right)\left(\left(s, s_{2}\right)\left(s, t_{2}\right)\right)=\frac{\mu_{\mathbf{A}_{1}}(s) \mu_{\mathbf{B}_{2}}\left(s_{2} t_{2}\right)}{\mu_{\mathbf{A}_{1}}(s)+\mu_{\mathbf{B}_{2}}\left(s_{2} t_{2}\right)-\mu_{\mathbf{A}_{1}}(s) \mu_{\mathbf{B}_{2}}\left(s_{2} t_{2}\right)} \\ \left(v_{\mathbf{B}_{1}} \square v_{\mathbf{B}_{2}}\right)\left(\left(s, s_{2}\right)\left(s, t_{2}\right)\right)=\frac{v_{\mathbf{A}_{1}}(s)+v_{\mathbf{B}_{2}}\left(s_{2} t_{2}\right)-2 v_{\mathbf{A}_{1}}(s) v_{\mathbf{B}_{2}}\left(s_{2} t_{2}\right)}{1-v_{\mathbf{A}_{1}}(s) v_{\mathbf{B}_{2}}\left(s_{2} t_{2}\right)}\end{array}\right.$ for all $s \in \mathbf{V}_{1}$ and $s_{2} t_{2} \in \mathbf{E}_{2}$,

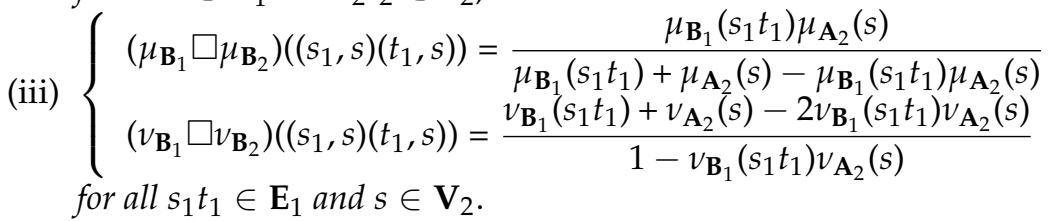

Remark 1. The Cartesian product $\mathbf{G}_{1} \square \mathbf{G}_{2}$ of two PDFGs $\mathbf{G}_{1}$ and $\mathbf{G}_{2}$ is not a PDFG as justified in the following example.

Consider PDFGs $\mathbf{G}_{1}$ and $\mathbf{G}_{2}$ as in Example 1. Then, the Cartesian product $\mathbf{G}_{1} \square \mathbf{G}_{2}$ is displayed in Figure 3.

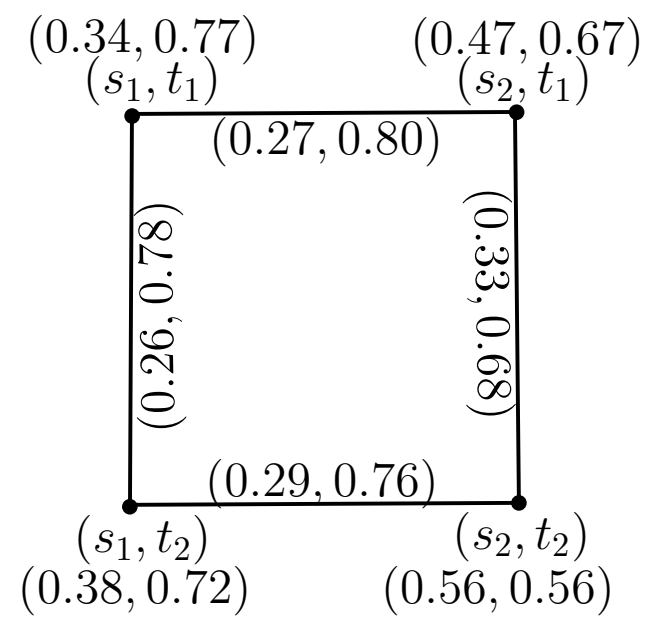

Figure 3. Cartesian product of two PDFGs.

Since for the membership and non-membership grade:

$$
\begin{gathered}
\left(\mu_{\mathbf{B}_{1}} \square \mu_{\mathbf{B}_{2}}\right)\left(\left(s_{1}, t_{1}\right)\left(s_{2}, t_{1}\right)\right)=0.27 \not \leq 0.25=\frac{\left(\mu_{\mathbf{A}_{1}} \square \mu_{\mathbf{A}_{2}}\right)\left(s_{1}, t_{1}\right)\left(\mu_{\mathbf{A}_{1}} \square \mu_{\mathbf{A}_{2}}\right)\left(s_{2}, t_{1}\right)}{\left(\mu_{\mathbf{A}_{1}} \square \mu_{\mathbf{A}_{2}}\right)\left(s_{1}, t_{1}\right)+\left(\mu_{\mathbf{A}_{1}} \square \mu_{\mathbf{A}_{2}}\right)\left(s_{2}, t_{1}\right)}, \\
-\left(\mu_{\mathbf{A}_{1}} \square \mu_{\mathbf{A}_{2}}\right)\left(s_{1}, t_{1}\right)\left(\mu_{\mathbf{A}_{1}} \square \mu_{\mathbf{A}_{2}}\right)\left(s_{2}, t_{1}\right)
\end{gathered}
$$

hence it is concluded that $\mathbf{G}_{1} \square \mathbf{G}_{2}=\left(\mathbf{A}_{1} \square \mathbf{A}_{2}, \mathbf{B}_{1} \square \mathbf{B}_{2}\right)$ is not a PDFG.

Definition 5. If the non-membership and membership grade of each edge of PDFG $\mathbf{G}$ of underlying crisp graph $\mathbf{G}^{\prime}$ is attached from zero and $[0,1]$, respectively, and each vertex is crisp in $\mathbf{G}$, then $\mathbf{G}$ is known as the Pythagorean Dombi fuzzy edge graph (PDFEG).

Proposition 4. If $\mathbf{G}_{1}=\left(\mathbf{A}_{1}, \mathbf{B}_{1}\right)$ and $\mathbf{G}_{2}=\left(\mathbf{A}_{2}, \mathbf{B}_{2}\right)$ are two PDFEGs of underlying crisp graphs $\mathbf{G}_{1}^{\prime}=$ $\left(\mathbf{V}_{1}, \mathbf{E}_{1}\right)$ and $\mathbf{G}_{2}^{\prime}=\left(\mathbf{V}_{2}, \mathbf{E}_{2}\right)$, respectively, then the Cartesian product $\mathbf{G}_{1} \square \mathbf{G}_{2}$ of $\mathbf{G}_{1}$ and $\mathbf{G}_{2}$ is the PDFEG of $\mathrm{G}_{1}^{\prime} \square \mathrm{G}_{2}^{\prime}$. 
Proof. Assume that $\mathrm{G}_{1}$ and $\mathrm{G}_{2}$ are two PDFEGs of underlying crisp graphs $\mathrm{G}_{1}^{\prime}$ and $\mathrm{G}_{2}^{\prime}$, respectively. Further, suppose that $\mathbf{G}=\mathbf{G}_{1} \square \mathbf{G}_{2}=\left(\mathbf{A}_{1} \square \mathbf{A}_{2}, \mathbf{B}_{1} \square \mathbf{B}_{2}\right)$ are the Cartesian product of $\mathbf{G}_{1}$ and $\mathbf{G}_{2}$, where $\mathbf{A}=\mathbf{A}_{1} \square \mathbf{A}_{2}$ and $\mathbf{B}=\mathbf{B}_{1} \square \mathbf{B}_{2}$ are the PF vertex and edge set, respectively. Let $\left(s_{1}, s_{2}\right)\left(t_{1}, t_{2}\right) \in \mathbf{E}_{1} \square \mathbf{E}_{2}$.

If $s_{1}=t_{1}=s$ and $s_{2} t_{2} \in \mathbf{E}_{2}$, then the membership and non-membership grade are:

$$
\begin{aligned}
& \left(\mu_{\mathbf{B}_{1}} \square \mu_{\mathbf{B}_{2}}\right)\left(\left(s, s_{2}\right)\left(s, t_{2}\right)\right)=\mathcal{T}\left(\mu_{\mathbf{A}_{1}}(s), \mu_{\mathbf{B}_{2}}\left(s_{2} t_{2}\right)\right)=\mathcal{T}\left(1, \mu_{\mathbf{B}_{2}}\left(s_{2} t_{2}\right)\right) \\
& =\mu_{\mathbf{B}_{2}}\left(s_{2} t_{2}\right) \leq \frac{\mu_{\mathbf{A}_{2}}\left(s_{2}\right) \mu_{\mathbf{A}_{2}}\left(t_{2}\right)}{\mu_{\mathbf{A}_{2}}\left(s_{2}\right)+\mu_{\mathbf{A}_{2}}\left(t_{2}\right)-\mu_{\mathbf{A}_{2}}\left(s_{2}\right) \mu_{\mathbf{A}_{2}}\left(t_{2}\right)} \\
& =\frac{\left(\mu_{\mathbf{A}_{1}} \square \mu_{\mathbf{A}_{2}}\right)\left(s, s_{2}\right)\left(\mu_{\mathbf{A}_{1}} \square \mu_{\mathbf{A}_{2}}\right)\left(s, t_{2}\right)}{\left(\mu_{\mathbf{A}_{1}} \square \mu_{\mathbf{A}_{2}}\right)\left(s, s_{2}\right)+\left(\mu_{\mathbf{A}_{1}} \square \mu_{\mathbf{A}_{2}}\right)\left(s, t_{2}\right)-\left(\mu_{\mathbf{A}_{1}} \square \mu_{\mathbf{A}_{2}}\right)\left(s, s_{2}\right)}, \\
& \left(\mu_{\mathbf{A}_{1}} \square \mu_{\mathbf{A}_{2}}\right)\left(s, t_{2}\right) \\
& \left(v_{\mathbf{B}_{1}} \square v_{\mathbf{B}_{2}}\right)\left(\left(s, s_{2}\right)\left(s, t_{2}\right)\right)=\mathcal{S}\left(v_{\mathbf{A}_{1}}(s), v_{\mathbf{B}_{2}}\left(s_{2} t_{2}\right)\right)=\mathcal{S}\left(0, v_{\mathbf{B}_{2}}\left(s_{2} t_{2}\right)\right) \\
& =v_{\mathbf{B}_{2}}\left(s_{2} t_{2}\right) \leq \frac{v_{\mathbf{A}_{2}}\left(s_{2}\right)+v_{\mathbf{A}_{2}}\left(t_{2}\right)-2 v_{\mathbf{A}_{2}}\left(s_{2}\right) v_{\mathbf{A}_{2}}\left(t_{2}\right)}{1-v_{\mathbf{A}_{2}}\left(s_{2}\right) v_{\mathbf{A}_{2}}\left(t_{2}\right)} \\
& \left(v_{\mathbf{A}_{1}} \square v_{\mathbf{A}_{2}}\right)\left(s, s_{2}\right)+\left(v_{\mathbf{A}_{1}} \square v_{\mathbf{A}_{2}}\right)\left(s, t_{2}\right)-2\left(v_{\mathbf{A}_{1}} \square v_{\mathbf{A}_{2}}\right)\left(s, s_{2}\right) \\
& =\frac{\left(v_{\mathbf{A}_{1}} \square v_{\mathbf{A}_{2}}\right)\left(s, t_{2}\right)}{1-\left(v_{\mathbf{A}_{1}} \square v_{\mathbf{A}_{2}}\right)\left(s, s_{2}\right)\left(v_{\mathbf{A}_{1}} \square v_{\mathbf{A}_{2}}\right)\left(s, t_{2}\right)} .
\end{aligned}
$$

If $s_{1} t_{1} \in \mathbf{E}_{1}$ and $s_{2}=t_{2}=s$, then the membership and non-membership grade are:

$$
\begin{aligned}
\left(\mu_{\mathbf{B}_{1}} \square \mu_{\mathbf{B}_{2}}\right)\left(\left(s_{1}, s\right)\left(t_{1}, s\right)\right)= & \mathcal{T}\left(\mu_{\mathbf{B}_{1}}\left(s_{1} t_{1}\right), \mu_{\mathbf{A}_{2}}(s)\right)=\mathcal{T}\left(\mu_{\mathbf{B}_{1}}\left(s_{1} t_{1}\right), 1\right) \\
= & \mu_{\mathbf{B}_{1}}\left(s_{1} t_{1}\right) \leq \frac{\mu_{\mathbf{A}_{1}}\left(s_{1}\right) \mu_{\mathbf{A}_{1}}\left(t_{1}\right)}{\mu_{\mathbf{A}_{1}}\left(s_{1}\right)+\mu_{\mathbf{A}_{1}}\left(t_{1}\right)-\mu_{\mathbf{A}_{1}}\left(s_{1}\right) \mu_{\mathbf{A}_{1}}\left(t_{1}\right)} \\
= & \frac{\left(\mu_{\mathbf{A}_{1}} \square \mu_{\mathbf{A}_{2}}\right)\left(s_{1}, s\right)\left(\mu_{\mathbf{A}_{1}} \square \mu_{\mathbf{A}_{2}}\right)\left(t_{1}, s\right)}{\left(\mu_{\mathbf{A}_{1}} \square \mu_{\mathbf{A}_{2}}\right)\left(s_{1}, s\right)+\left(\mu_{\mathbf{A}_{1}} \square \mu_{\mathbf{A}_{2}}\right)\left(t_{1}, s\right)-\left(\mu_{\mathbf{A}_{1}} \square \mu_{\mathbf{A}_{2}}\right)\left(s_{1}, s\right)}, \\
\left(v_{\mathbf{B}_{1}} \square v_{\mathbf{B}_{2}}\right)\left(\left(s_{1}, s\right)\left(t_{\mathbf{A}_{2}}\right)\left(t_{1}, s\right)\right) & =\mathcal{S}\left(v_{\mathbf{B}_{1}}\left(s_{1} t_{1}\right), v_{\mathbf{A}_{2}}(s)\right)=\mathcal{S}\left(v_{\mathbf{B}_{1}}\left(s_{1} t_{1}\right), 0\right) \\
= & v_{\mathbf{B}_{1}}\left(s_{1} t_{1}\right) \leq \frac{v_{\mathbf{A}_{1}}\left(s_{1}\right)+v_{\mathbf{A}_{1}}\left(t_{1}\right)-2 v_{\mathbf{A}_{1}}\left(s_{1}\right) v_{\mathbf{A}_{1}}\left(t_{1}\right)}{1-v_{\mathbf{A}_{1}}\left(s_{1}\right) v_{\mathbf{A}_{1}}\left(t_{1}\right)} \\
& \left(v_{\mathbf{A}_{1}} \square v_{\mathbf{A}_{2}}\right)\left(s_{1}, s\right)+\left(v_{\mathbf{A}_{1}} \square v_{\mathbf{A}_{2}}\right)\left(t_{1}, s\right)-2\left(v_{\mathbf{A}_{1}} \square v_{\mathbf{A}_{2}}\right)\left(s_{1}, s\right) \\
= & \frac{\left(v_{\mathbf{A}_{1}} \square v_{\mathbf{A}_{2}}\right)\left(t_{1}, s\right)}{1-\left(v_{\mathbf{A}_{1}} \square v_{\mathbf{A}_{2}}\right)\left(s_{1}, s\right)\left(v_{\mathbf{A}_{1}} \square v_{\mathbf{A}_{2}}\right)\left(t_{1}, s\right)} .
\end{aligned}
$$

Hence, it is concluded that $\mathbf{G}_{1} \square \mathbf{G}_{2}$ is a PDFEG of $\mathbf{G}_{1}^{\prime} \square \mathbf{G}_{2}^{\prime}$.

Example 3. Consider PDFEGs $\mathbf{G}_{1}=\left(\mathbf{A}_{1}, \mathbf{B}_{1}\right)$ and $\mathbf{G}_{2}=\left(\mathbf{A}_{2}, \mathbf{B}_{2}\right)$ as represented in Figure 4, such that $\mu_{\mathbf{A}_{1}}\left(s_{i}\right)=1, v_{\mathbf{A}_{1}}\left(s_{i}\right)=0$ for all $s_{i} \in \mathbf{V}_{1}$ and $i=1,2$, where $\mu_{\mathbf{B}_{1}}=\left\{\frac{s_{1} s_{2}}{0.8}\right\}, v_{\mathbf{B}_{1}}=\left\{\frac{s_{1} s_{2}}{0.0}\right\}$ and $\mu_{\mathbf{A}_{2}}\left(t_{j}\right)=1$, $v_{\mathbf{A}_{2}}\left(t_{j}\right)=0$ for all $t_{j} \in \mathbf{V}_{2}$ and $j=1,2$, where $\mu_{\mathbf{B}_{2}}=\left\{\frac{t_{1} t_{2}}{0.7}\right\}, v_{\mathbf{B}_{2}}=\left\{\frac{t_{1} t_{2}}{0.0}\right\}$. Then, $\mathbf{G}_{1} \square \mathbf{G}_{2}$ is given in Figure 5 . 


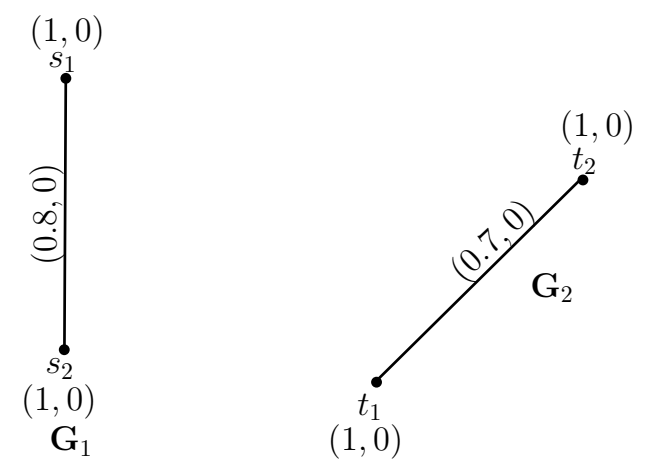

Figure 4. Pythagorean Dombi fuzzy edge graphs.

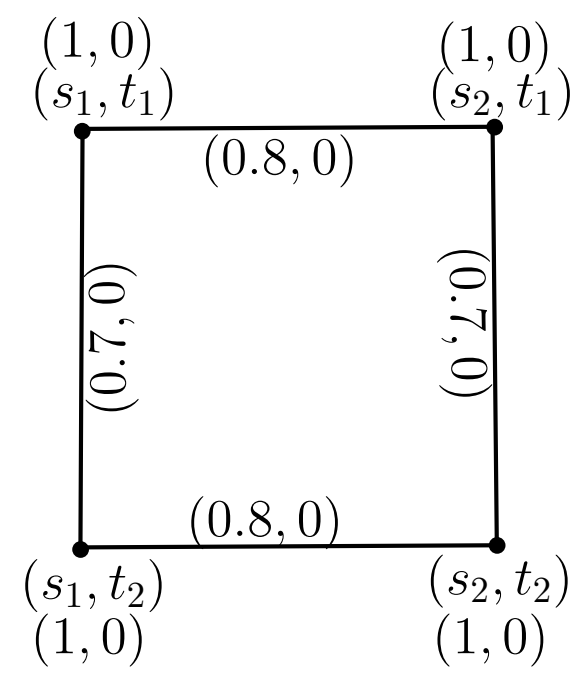

Figure 5. Cartesian product of two PDFEGs.

By routine computations, one can view from Figure 5 that $\mathbf{G}_{1} \square \mathbf{G}_{2}=\left(\mathbf{A}_{1} \square \mathbf{A}_{2}, \mathbf{B}_{1} \square \mathbf{B}_{2}\right)$ is a PDFEG of the underlying crisp graph $\mathbf{G}_{1}^{\prime} \square \mathbf{G}_{2}^{\prime}=\left(\mathbf{V}_{1} \square \mathbf{V}_{2}, \mathbf{E}_{1} \square \mathbf{E}_{2}\right)$.

Definition 6. Consider $\mathbf{G}_{1}=\left(\mathbf{A}_{1}, \mathbf{B}_{1}\right)$ and $\mathbf{G}_{2}=\left(\mathbf{A}_{2}, \mathbf{B}_{2}\right)$ to be two PDFEGs. Then, for any vertex $\left(s_{1}, s_{2}\right) \in$ $\mathbf{V}_{1} \square \mathbf{V}_{2}$,

$$
\begin{aligned}
\left(\mathbf{D}_{\mu}\right)_{\mathbf{G}_{1} \square \mathbf{G}_{2}}\left(s_{1}, s_{2}\right)= & \sum_{\left(s_{1}, s_{2}\right)\left(t_{1}, t_{2}\right) \in \mathbf{E}_{1} \square \mathbf{E}_{2}}\left(\mu_{\mathbf{B}_{1}} \square \mu_{\mathbf{B}_{2}}\right)\left(\left(s_{1}, s_{2}\right)\left(t_{1}, t_{2}\right)\right) \\
= & \sum_{s_{1}=t_{1}, s_{2} t_{2} \in \mathbf{E}_{2}} \frac{\mu_{\mathbf{A}_{1}}\left(s_{1}\right) \mu_{\mathbf{B}_{2}}\left(s_{2} t_{2}\right)}{\mu_{\mathbf{A}_{1}}\left(s_{1}\right)+\mu_{\mathbf{B}_{2}}\left(s_{2} t_{2}\right)-\mu_{\mathbf{A}_{1}}\left(s_{1}\right) \mu_{\mathbf{B}_{2}}\left(s_{2} t_{2}\right)} \\
& +\sum_{s_{1} t_{1} \in \mathbf{E}_{1}, s_{2}=t_{2}} \frac{\mu_{\mathbf{B}_{1}}\left(s_{1} t_{1}\right) \mu_{\mathbf{A}_{2}}\left(s_{2}\right)}{\mu_{\mathbf{B}_{1}}\left(s_{1} t_{1}\right)+\mu_{\mathbf{A}_{2}}\left(s_{2}\right)-\mu_{\mathbf{B}_{1}}\left(s_{1} t_{1}\right) \mu_{\mathbf{A}_{2}}\left(s_{2}\right)},
\end{aligned}
$$




$$
\begin{aligned}
\left(\mathbf{D}_{v}\right)_{\mathbf{G}_{1} \square \mathbf{G}_{2}}\left(s_{1}, s_{2}\right)= & \sum_{\left(s_{1}, s_{2}\right)\left(t_{1}, t_{2}\right) \in \mathbf{E}_{1} \square \mathbf{E}_{2}}\left(v_{\mathbf{B}_{1}} \square v_{\mathbf{B}_{2}}\right)\left(\left(s_{1}, s_{2}\right)\left(t_{1}, t_{2}\right)\right) \\
= & \sum_{s_{1}=t_{1}, s_{2} t_{2} \in \mathbf{E}_{2}} \frac{v_{\mathbf{A}_{1}}\left(s_{1}\right)+v_{\mathbf{B}_{2}}\left(s_{2} t_{2}\right)-2 v_{\mathbf{A}_{1}}\left(s_{1}\right) v_{\mathbf{B}_{2}}\left(s_{2} t_{2}\right)}{1-v_{\mathbf{A}_{1}}\left(s_{1}\right) v_{\mathbf{B}_{2}}\left(s_{2} t_{2}\right)} \\
& +\sum_{s_{1} t_{1} \in \mathbf{E}_{1}, s_{2}=t_{2}} \frac{v_{\mathbf{B}_{1}}\left(s_{1} t_{1}\right)+v_{\mathbf{A}_{2}}\left(s_{2}\right)-2 v_{\mathbf{B}_{1}}\left(s_{1} t_{1}\right) v_{\mathbf{A}_{2}}\left(s_{2}\right)}{1-v_{\mathbf{B}_{1}}\left(s_{1} t_{1}\right) v_{\mathbf{A}_{2}}\left(s_{2}\right)} .
\end{aligned}
$$

Definition 7. Consider $\mathbf{G}_{1}=\left(\mathbf{A}_{1}, \mathbf{B}_{1}\right)$ and $\mathbf{G}_{2}=\left(\mathbf{A}_{2}, \mathbf{B}_{2}\right)$ to be two PDFEGs. Then, for any vertex $\left(s_{1}, s_{2}\right) \in$ $\mathbf{V}_{1} \square \mathbf{V}_{2}$,

$$
\begin{aligned}
\left(\mathbf{T D}_{\mu}\right)_{\mathbf{G}_{1} \square \mathbf{G}_{2}}\left(s_{1}, s_{2}\right)= & \sum_{\left(s_{1}, s_{2}\right)\left(t_{1}, t_{2}\right) \in \mathbf{E}_{1} \square \mathbf{E}_{2}}\left(\mu_{\mathbf{B}_{1}} \square \mu_{\mathbf{B}_{2}}\right)\left(\left(s_{1}, s_{2}\right)\left(t_{1}, t_{2}\right)\right)+\left(\mu_{\mathbf{A}_{1}} \square \mu_{\mathbf{A}_{2}}\right)\left(s_{1}, s_{2}\right) \\
= & \sum_{s_{1}=t_{1}, s_{2} t_{2} \in \mathbf{E}_{2}} \frac{\mu_{\mathbf{A}_{1}}\left(s_{1}\right) \mu_{\mathbf{B}_{2}}\left(s_{2} t_{2}\right)}{\mu_{\mathbf{A}_{1}}\left(s_{1}\right)+\mu_{\mathbf{B}_{2}}\left(s_{2} t_{2}\right)-\mu_{\mathbf{A}_{1}}\left(s_{1}\right) \mu_{\mathbf{B}_{2}}\left(s_{2} t_{2}\right)} \\
& +\sum_{s_{1} t_{1} \in \mathbf{E}_{1}, s_{2}=t_{2}} \frac{\mu_{\mathbf{B}_{1}}\left(s_{1} t_{1}\right)+\mu_{\mathbf{A}_{2}}\left(s_{2}\right)-\mu_{\mathbf{B}_{1}}\left(s_{1} t_{1}\right) \mu_{\mathbf{A}_{2}}\left(s_{2}\right)}{\mu_{\mathbf{A}_{1}}\left(s_{1}\right) \mu_{\mathbf{A}_{2}}\left(s_{2}\right)} \\
& +\frac{\mu_{\mathbf{B}_{1}}\left(s_{1}\right) \mu_{\mathbf{A}_{1}}\left(s_{1}\right)+\mu_{\mathbf{A}_{2}}\left(s_{2}\right)-\mu_{\mathbf{A}_{1}}\left(s_{1}\right) \mu_{\mathbf{A}_{2}}\left(s_{2}\right)}{\mu_{\mathbf{A}_{1}}}, \\
\left(\mathbf{T D}_{v}\right)_{\mathbf{G}_{1} \square \mathbf{G}_{2}}\left(s_{1}, s_{2}\right)= & \sum_{\left(s_{1}, s_{2}\right)\left(t_{1}, t_{2}\right) \in \mathbf{E}_{1} \square \mathbf{E}_{2}}\left(v_{\mathbf{B}_{1}} \square v_{\mathbf{B}_{2}}\right)\left(\left(s_{1}, s_{2}\right)\left(t_{1}, t_{2}\right)\right)+\left(v_{\mathbf{A}_{1}} \square v_{\mathbf{A}_{2}}\right)\left(s_{1}, s_{2}\right) \\
= & \sum_{s_{1}=t_{1}, s_{2} t_{2} \in \mathbf{E}_{2}} \frac{v_{\mathbf{A}_{1}}\left(s_{1}\right)+v_{\mathbf{B}_{2}}\left(s_{2} t_{2}\right)-2 v_{\mathbf{A}_{1}}\left(s_{1}\right) v_{\mathbf{B}_{2}}\left(s_{2} t_{2}\right)}{1-v_{\mathbf{A}_{1}}\left(s_{1}\right) v_{\mathbf{B}_{2}}\left(s_{2} t_{2}\right)} \\
& +\sum_{s_{1} t_{1} \in \mathbf{E}_{1}, s_{2}=t_{2}} \frac{v_{\mathbf{B}_{1}}\left(s_{1} t_{1}\right)+v_{\mathbf{A}_{2}}\left(s_{2}\right)-2 v_{\mathbf{B}_{1}}\left(s_{1} t_{1}\right) v_{\mathbf{A}_{2}}\left(s_{2}\right)}{1-v_{\mathbf{B}_{1}}\left(s_{1} t_{1}\right) v_{\mathbf{A}_{2}}\left(s_{2}\right)} \\
& +\frac{v_{\mathbf{A}_{1}}\left(s_{1}\right)+v_{\mathbf{A}_{2}}\left(s_{2}\right)-2 v_{\mathbf{A}_{1}}\left(s_{1}\right) v_{\mathbf{A}_{2}}\left(s_{2}\right)}{1-v_{\mathbf{A}_{1}}\left(s_{1}\right) v_{\mathbf{A}_{2}}\left(s_{2}\right)} .
\end{aligned}
$$

Example 4. Consider PDFEGs $\mathbf{G}_{1}$ and $\mathbf{G}_{2}$ as in Example 3; their $\mathbf{G}_{1} \square \mathbf{G}_{2}$ is given in Figure 5. Then, by Definition 6, we must have:

$$
\begin{aligned}
& \left(\mathbf{D}_{\mu}\right)_{\mathbf{G}_{1} \square \mathbf{G}_{2}}\left(s_{1}, t_{1}\right)=\quad\left(\mu_{\mathbf{B}_{1}} \square \mu_{\mathbf{B}_{2}}\right)\left(\left(s_{1}, t_{1}\right)\left(s_{2}, t_{1}\right)\right)+\left(\mu_{\mathbf{B}_{1}} \square \mu_{\mathbf{B}_{2}}\right)\left(\left(s_{1}, t_{1}\right)\left(s_{1}, t_{2}\right)\right) \\
& =\frac{\mu_{\mathbf{B}_{1}}\left(s_{1} s_{2}\right) \mu_{\mathbf{A}_{2}}\left(t_{1}\right)}{\mu_{\mathbf{B}_{1}}\left(s_{1} s_{2}\right)+\mu_{\mathbf{A}_{2}}\left(t_{1}\right)-\mu_{\mathbf{B}_{1}}\left(s_{1} s_{2}\right) \mu_{\mathbf{A}_{2}}\left(t_{1}\right)}+\frac{\mu_{\mathbf{A}_{1}}\left(s_{1}\right) \mu_{\mathbf{B}_{2}}\left(t_{1} t_{2}\right)}{\mu_{\mathbf{A}_{1}}\left(s_{1}\right)+\mu_{\mathbf{B}_{2}}\left(t_{1} t_{2}\right)-\mu_{\mathbf{A}_{1}}\left(s_{1}\right) \mu_{\mathbf{B}_{2}}\left(t_{1} t_{2}\right)}=1.5, \\
& \left(\mathbf{D}_{v}\right)_{\mathbf{G}_{1} \square \mathbf{G}_{2}}\left(s_{1}, t_{1}\right)=\quad\left(v_{\mathbf{B}_{1}} \square v_{\mathbf{B}_{2}}\right)\left(\left(s_{1}, t_{1}\right)\left(s_{2}, t_{1}\right)\right)+\left(v_{\mathbf{B}_{1}} \square v_{\mathbf{B}_{2}}\right)\left(\left(s_{1}, t_{1}\right)\left(s_{1}, t_{2}\right)\right) \\
& =\frac{v_{\mathbf{B}_{1}}\left(s_{1} s_{2}\right)+v_{\mathbf{A}_{2}}\left(t_{1}\right)-2 v_{\mathbf{B}_{1}}\left(s_{1} s_{2}\right) v_{\mathbf{A}_{2}}\left(t_{1}\right)}{1-v_{\mathbf{B}_{1}}\left(s_{1} s_{2}\right) v_{\mathbf{A}_{2}}\left(t_{1}\right)}+\frac{v_{\mathbf{A}_{1}}\left(s_{1}\right)+v_{\mathbf{B}_{2}}\left(t_{1} t_{2}\right)-2 v_{\mathbf{A}_{1}}\left(s_{1}\right) v_{\mathbf{B}_{2}}\left(t_{1} t_{2}\right)}{1-v_{\mathbf{A}_{1}}\left(s_{1}\right) v_{\mathbf{B}_{2}}\left(t_{1} t_{2}\right)}=0 .
\end{aligned}
$$

Thus, $(\mathbf{D})_{\mathbf{G}_{1} \square \mathbf{G}_{2}}\left(s_{1}, t_{1}\right)=(1.5,0)$.

Further, by using Definition 7, we have:

$$
\begin{aligned}
\left(\mathbf{T D}_{\mu}\right)_{\mathbf{G}_{1} \square \mathbf{G}_{2}}\left(s_{1}, t_{1}\right) & =\left(\mathbf{D}_{\mu}\right)_{\mathbf{G}_{1} \square \mathbf{G}_{2}}\left(s_{1}, t_{1}\right)+\left(\mu_{\mathbf{A}_{1}} \square \mu_{\mathbf{A}_{2}}\right)\left(s_{1}, t_{1}\right) \\
& =1.5+\frac{\mu_{\mathbf{A}_{1}}\left(s_{1}\right) \mu_{\mathbf{A}_{2}}\left(t_{1}\right)}{\mu_{\mathbf{A}_{1}}\left(s_{1}\right)+\mu_{\mathbf{A}_{2}}\left(t_{1}\right)-\mu_{\mathbf{A}_{1}}\left(s_{1}\right) \mu_{\mathbf{A}_{2}}\left(t_{1}\right)}=2.5, \\
\left(\mathbf{T D}_{v}\right)_{\mathbf{G}_{1} \square \mathbf{G}_{2}}\left(s_{1}, t_{1}\right) & =\left(\mathbf{D}_{v}\right)_{\mathbf{G}_{1} \square \mathbf{G}_{2}}\left(s_{1}, t_{1}\right)+\left(v_{\mathbf{A}_{1}} \square v_{\mathbf{A}_{2}}\right)\left(s_{1}, t_{1}\right) \\
& =0+\frac{v_{\mathbf{A}_{1}}\left(s_{1}\right)+v_{\mathbf{A}_{2}}\left(t_{1}\right)-2 v_{\mathbf{A}_{1}}\left(s_{1}\right) v_{\mathbf{A}_{2}}\left(t_{1}\right)}{1-v_{\mathbf{A}_{1}}\left(s_{1}\right) v_{\mathbf{A}_{2}}\left(t_{1}\right)}=0 .
\end{aligned}
$$


Thus, (TD $)_{\mathbf{G}_{1} \square \mathbf{G}_{2}}\left(s_{1}, t_{1}\right)=(2.5,0)$.

Remark 2. The Cartesian product $\mathbf{G}_{1} \square \mathbf{G}_{2}$ of strong PDFGs $\mathbf{G}_{1}$ and $\mathbf{G}_{2}$ is not a PDFG. This is justified in the following example.

Consider strong PDFGs $\mathbf{G}_{1}$ and $\mathbf{G}_{2}$. Their $\mathbf{G}_{1} \square \mathbf{G}_{2}$ is displayed in Figure 6 .

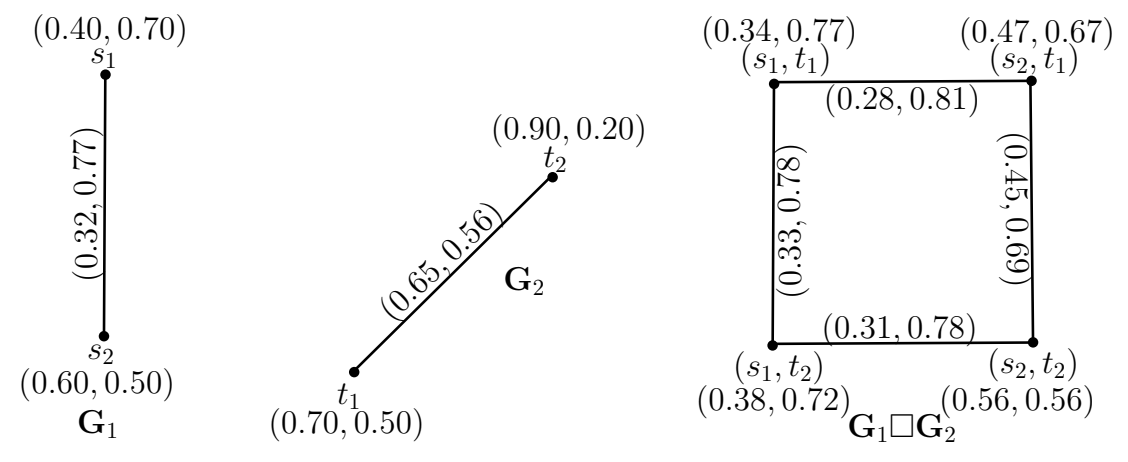

Figure 6. $\mathrm{G}_{1} \square \mathrm{G}_{2}$ is not a PDFG.

Since for the membership and non-membership grade of $\left(s_{1}, t_{1}\right)\left(s_{1}, t_{2}\right)$ :

$$
\begin{aligned}
\left(\mu_{\mathbf{B}_{1}} \square \mu_{\mathbf{B}_{2}}\right)\left(\left(s_{1}, t_{1}\right)\left(s_{1}, t_{2}\right)\right)=0.33 \not \leq 0.22= & \frac{\left(\mu_{\mathbf{A}_{1}} \square \mu_{\mathbf{A}_{2}}\right)\left(s_{1}, t_{1}\right)\left(\mu_{\mathbf{A}_{1}} \square \mu_{\mathbf{A}_{2}}\right)\left(s_{1}, t_{2}\right)}{\left(\mu_{\mathbf{A}_{1}} \square \mu_{\mathbf{A}_{2}}\right)\left(s_{1}, t_{1}\right)+\left(\mu_{\mathbf{A}_{1}} \square \mu_{\mathbf{A}_{2}}\right)\left(s_{1}, t_{2}\right)}, \\
& -\left(\mu_{\mathbf{A}_{1}} \square \mu_{\mathbf{A}_{2}}\right)\left(s_{1}, t_{1}\right)\left(\mu_{\mathbf{A}_{1}} \square \mu_{\mathbf{A}_{2}}\right)\left(s_{1}, t_{2}\right) \\
& \left(v_{\mathbf{A}_{1}} \square v_{\mathbf{A}_{2}}\right)\left(s_{1}, t_{1}\right)+\left(v_{\mathbf{A}_{1}} \square v_{\mathbf{A}_{2}}\right)\left(s_{1}, t_{2}\right) \\
\left(v_{\mathbf{B}_{1}} \square v_{\mathbf{B}_{2}}\right)\left(\left(s_{1}, t_{1}\right)\left(s_{1}, t_{2}\right)\right)=0.78 \leq 0.86= & \frac{-2\left(v_{\mathbf{A}_{1}} \square v_{\mathbf{A}_{2}}\right)\left(s_{1}, t_{1}\right)\left(v_{\mathbf{A}_{1}} \square v_{\mathbf{A}_{2}}\right)\left(s_{1}, t_{2}\right)}{1-\left(v_{\mathbf{A}_{1}} \square v_{\mathbf{A}_{2}}\right)\left(s_{1}, t_{1}\right)\left(v_{\mathbf{A}_{1}} \square v_{\mathbf{A}_{2}}\right)\left(s_{1}, t_{2}\right)} .
\end{aligned}
$$

Likewise, for the membership and non-membership grade of $\left(s_{1}, t_{1}\right)\left(s_{2}, t_{1}\right)$ :

$$
\begin{aligned}
& \left(\mu_{\mathbf{B}_{1}} \square \mu_{\mathbf{B}_{2}}\right)\left(\left(s_{1}, t_{1}\right)\left(s_{2}, t_{1}\right)\right)=0.28 \not \leq 0.25=\frac{\left(\mu_{\mathbf{A}_{1}} \square \mu_{\mathbf{A}_{2}}\right)\left(s_{1}, t_{1}\right)\left(\mu_{\mathbf{A}_{1}} \square \mu_{\mathbf{A}_{2}}\right)\left(s_{2}, t_{1}\right)}{\left(\mu_{\mathbf{A}_{1}} \square \mu_{\mathbf{A}_{2}}\right)\left(s_{1}, t_{1}\right)+\left(\mu_{\mathbf{A}_{1}} \square \mu_{\mathbf{A}_{2}}\right)\left(s_{2}, t_{1}\right)}, \\
& -\left(\mu_{\mathbf{A}_{1}} \square \mu_{\mathbf{A}_{2}}\right)\left(s_{1}, t_{1}\right)\left(\mu_{\mathbf{A}_{1}} \square \mu_{\mathbf{A}_{2}}\right)\left(s_{2}, t_{1}\right) \\
& \left(v_{\mathbf{A}_{1}} \square v_{\mathbf{A}_{2}}\right)\left(s_{1}, t_{1}\right)+\left(v_{\mathbf{A}_{1}} \square v_{\mathbf{A}_{2}}\right)\left(s_{2}, t_{1}\right) \\
& \left(v_{\mathbf{B}_{1}} \square v_{\mathbf{B}_{2}}\right)\left(\left(s_{1}, t_{1}\right)\left(s_{2}, t_{1}\right)\right)=0.81 \leq 0.84=\frac{-2\left(v_{\mathbf{A}_{1}} \square v_{\mathbf{A}_{2}}\right)\left(s_{1}, t_{1}\right)\left(v_{\mathbf{A}_{1}} \square v_{\mathbf{A}_{2}}\right)\left(s_{2}, t_{1}\right)}{1-\left(v_{\mathbf{A}_{1}} \square v_{\mathbf{A}_{2}}\right)\left(s_{1}, t_{1}\right)\left(v_{\mathbf{A}_{1}} \square v_{\mathbf{A}_{2}}\right)\left(s_{2}, t_{1}\right)} .
\end{aligned}
$$

Hence, it is concluded that $\mathbf{G}_{1} \square \mathbf{G}_{2}$ is not a PDFG of underlying crisp graph $\mathbf{G}_{1}^{\prime} \square \mathbf{G}_{2}^{\prime}$.

Proposition 5. If $\mathbf{G}_{1}=\left(\mathbf{A}_{1}, \mathbf{B}_{1}\right)$ and $\mathbf{G}_{2}=\left(\mathbf{A}_{2}, \mathbf{B}_{2}\right)$ are strong PDFEGs of underlying crisp graphs $\mathbf{G}_{1}^{\prime}=$ $\left(\mathbf{V}_{1}, \mathbf{E}_{1}\right)$ and $\mathbf{G}_{2}^{\prime}=\left(\mathbf{V}_{2}, \mathbf{E}_{2}\right)$, respectively, then the Cartesian product $\mathbf{G}_{1} \square \mathbf{G}_{2}$ of $\mathbf{G}_{1}$ and $\mathbf{G}_{2}$ is also a strong PDFEG of $\mathbf{G}_{1}^{\prime} \square \mathbf{G}_{2}^{\prime}$.

Proof. The proof is the same as Proposition 4. 


\subsection{Semi-Strong Product of Pythagorean Dombi Fuzzy Graphs}

Definition 8. Let $\mathbf{A}_{j}$ and $\mathbf{B}_{j}$ be the Pythagorean fuzzy subsets of $\mathbf{V}_{j}$ and $\mathbf{E}_{j}(j=1,2)$, respectively. The semi-strong product of PDFGs $\mathbf{G}_{1}=\left(\mathbf{A}_{1}, \mathbf{B}_{1}\right)$ and $\mathbf{G}_{2}=\left(\mathbf{A}_{2}, \mathbf{B}_{2}\right)$ of underlying crisp graphs $\mathbf{G}_{1}^{\prime}=\left(\mathbf{V}_{1}, \mathbf{E}_{1}\right)$ and $\mathbf{G}_{2}^{\prime}=\left(\mathbf{V}_{2}, \mathbf{E}_{2}\right)$, respectively, is represented by $\mathbf{G}_{1} \bullet \mathbf{G}_{2}=\left(\mathbf{A}_{1} \bullet \mathbf{A}_{2}, \mathbf{B}_{1} \bullet \mathbf{B}_{2}\right)$, defined as:

(i)

(i) $\left\{\begin{array}{l}\left(\mu_{\mathbf{A}_{1}} \bullet \mu_{\mathbf{A}_{2}}\right)\left(s_{1}, s_{2}\right)=\frac{\mu_{\mathbf{A}_{1}}\left(s_{1}\right) \mu_{\mathbf{A}_{2}}\left(s_{2}\right)}{\mu_{\mathbf{A}_{1}}\left(s_{1}\right)+\mu_{\mathbf{A}_{2}}\left(s_{2}\right)-\mu_{\mathbf{A}_{1}}\left(s_{1}\right) \mu_{\mathbf{A}_{2}}\left(s_{2}\right)} \\ \left(v_{\mathbf{A}_{1}} \bullet v_{\mathbf{A}_{2}}\right)\left(s_{1}, s_{2}\right)=\frac{v_{\mathbf{A}_{1}}\left(s_{1}\right)+v_{\mathbf{A}_{2}}\left(s_{2}\right)-2 v_{\mathbf{A}_{1}}\left(s_{1}\right) v_{\mathbf{A}_{2}}\left(s_{2}\right)}{1-v_{\mathbf{A}_{1}}\left(s_{1}\right) v_{\mathbf{A}_{2}}\left(s_{2}\right)}\end{array}\right.$ for all $\left(s_{1}, s_{2}\right) \in \mathbf{V}_{1} \bullet \mathbf{V}_{2}$,

(ii) $\left\{\begin{aligned}\left(\mu_{\mathbf{B}_{1}} \bullet \mu_{\mathbf{B}_{2}}\right)\left(\left(s, s_{2}\right)\left(s, t_{2}\right)\right) & =\frac{\mu_{\mathbf{A}_{1}}(s) \mu_{\mathbf{B}_{2}}\left(s_{2} t_{2}\right)}{\mu_{\mathbf{A}_{1}}(s)+\mu_{\mathbf{B}_{2}}\left(s_{2} t_{2}\right)-\mu_{\mathbf{A}_{1}}(s) \mu_{\mathbf{B}_{2}}\left(s_{2} t_{2}\right)} \\ \left(v_{\mathbf{B}_{1}} \bullet v_{\mathbf{B}_{2}}\right)\left(\left(s, s_{2}\right)\left(s, t_{2}\right)\right) & =\frac{v_{\mathbf{A}_{1}}(s)+v_{\mathbf{B}_{2}}\left(s_{2} t_{2}\right)-2 v_{\mathbf{A}_{1}}(s) v_{\mathbf{B}_{2}}\left(s_{2} t_{2}\right)}{1-v_{\mathbf{A}_{1}}(s) v_{\mathbf{B}_{2}}\left(s_{2} t_{2}\right)}\end{aligned}\right.$ for all $s \in \mathbf{V}_{1}$ and $s_{2} t_{2} \in \mathbf{E}_{2}$,

(iii) $\left\{\begin{array}{l}\left(\mu_{\mathbf{B}_{1}} \bullet \mu_{\mathbf{B}_{2}}\right)\left(\left(s_{1}, s_{2}\right)\left(t_{1}, t_{2}\right)\right)=\frac{\mu_{\mathbf{B}_{1}}\left(s_{1} t_{1}\right) \mu_{\mathbf{B}_{2}}\left(s_{2} t_{2}\right)}{\mu_{\mathbf{B}_{1}}\left(s_{1} t_{1}\right)+\mu_{\mathbf{B}_{2}}\left(s_{2} t_{2}\right)-\mu_{\mathbf{B}_{1}}\left(s_{1} t_{1}\right) \mu_{\mathbf{B}_{2}}\left(s_{2} t_{2}\right)} \\ \left(v_{\mathbf{B}_{1}} \bullet v_{\mathbf{B}_{2}}\right)\left(\left(s_{1}, s_{2}\right)\left(t_{1}, t_{2}\right)\right)=\frac{v_{\mathbf{B}_{1}}\left(s_{1} t_{1}\right)+v_{\mathbf{B}_{2}}\left(s_{2} t_{2}\right)-2 v_{\mathbf{B}_{1}}\left(s_{1} t_{1}\right) v_{\mathbf{B}_{2}}\left(s_{2} t_{2}\right)}{1-v_{\mathbf{B}_{1}}\left(s_{1} t_{1}\right) v_{\mathbf{B}_{2}}\left(s_{2} t_{2}\right)}\end{array}\right.$ for all $s_{1} t_{1} \in \mathbf{E}_{1}$ and $s_{2} t_{2} \in \mathbf{E}_{2}$.

Proposition 6. If $\mathbf{G}_{1}=\left(\mathbf{A}_{1}, \mathbf{B}_{1}\right)$ and $\mathbf{G}_{2}=\left(\mathbf{A}_{2}, \mathbf{B}_{2}\right)$ are PDFEGs of underlying crisp graphs $\mathbf{G}_{1}^{\prime}=\left(\mathbf{V}_{1}, \mathbf{E}_{1}\right)$ and $\mathbf{G}_{2}^{\prime}=\left(\mathbf{V}_{2}, \mathbf{E}_{2}\right)$, respectively, then the semi-strong product $\mathbf{G}_{1} \bullet \mathbf{G}_{2}$ of $\mathbf{G}_{1}$ and $\mathbf{G}_{2}$ is the PDFEG of $\mathbf{G}_{1}^{\prime} \bullet \mathbf{G}_{2}^{\prime}$.

Proof. This proposition can be easily proven in the same way as Proposition 1 and Proposition 4 were proven.

Example 5. Consider PDFEGs $\mathbf{G}_{1}$ and $\mathbf{G}_{2}$ as in Example 3. Then, $\mathbf{G}_{1} \bullet \mathbf{G}_{2}$ is displayed in Figure 7.

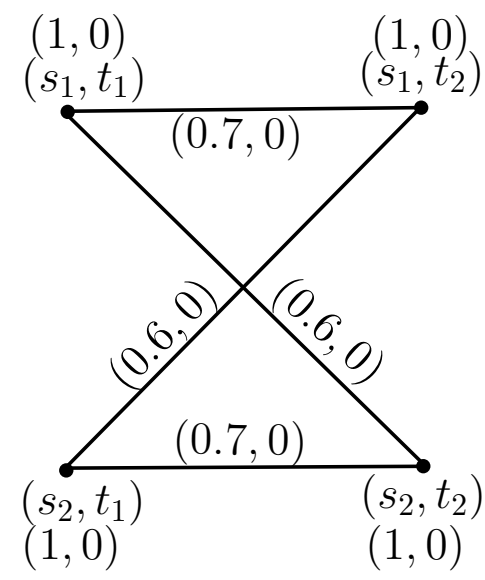

Figure 7. Semi-strong product of two PDFEGs.

By routine computations, one can view from Figure 7 that $\mathbf{G}_{1} \bullet \mathbf{G}_{2}=\left(\mathbf{A}_{1} \bullet \mathbf{A}_{2}, \mathbf{B}_{1} \bullet \mathbf{B}_{2}\right)$ is a PDFEG of underlying crisp graph $\mathbf{G}_{1}^{\prime} \bullet \mathbf{G}_{2}^{\prime}=\left(\mathbf{V}_{1} \bullet \mathbf{V}_{2}, \mathbf{E}_{1} \bullet \mathbf{E}_{2}\right)$. 
Definition 9. Consider $\mathbf{G}_{1}=\left(\mathbf{A}_{1}, \mathbf{B}_{1}\right)$ and $\mathbf{G}_{2}=\left(\mathbf{A}_{2}, \mathbf{B}_{2}\right)$ to be two PDFEGs. Then, for any vertex $\left(s_{1}, s_{2}\right) \in$ $\mathbf{V}_{1} \bullet \mathbf{V}_{2}$,

$$
\begin{aligned}
\left(\mathbf{D}_{\mu}\right)_{\mathbf{G}_{1} \bullet \mathbf{G}_{2}}\left(s_{1}, s_{2}\right)= & \sum_{\left(s_{1}, s_{2}\right)\left(t_{1}, t_{2}\right) \in \mathbf{E}_{1} \bullet E_{2}}\left(\mu_{\mathbf{B}_{1}} \bullet \mu_{\mathbf{B}_{2}}\right)\left(\left(s_{1}, s_{2}\right)\left(t_{1}, t_{2}\right)\right) \\
= & \sum_{s_{1}=t_{1}, s_{2} t_{2} \in \mathbf{E}_{2}} \frac{\mu_{\mathbf{A}_{1}}\left(s_{1}\right) \mu_{\mathbf{B}_{2}}\left(s_{2} t_{2}\right)}{\mu_{\mathbf{A}_{1}}\left(s_{1}\right)+\mu_{\mathbf{B}_{2}}\left(s_{2} t_{2}\right)-\mu_{\mathbf{A}_{1}}\left(s_{1}\right) \mu_{\mathbf{B}_{2}}\left(s_{2} t_{2}\right)} \\
& +\sum_{s_{1} t_{1} \in \mathbf{E}_{1}, s_{2} t_{2} \in \mathbf{E}_{2}} \frac{\mu_{\mathbf{B}_{1}}\left(s_{1} t_{1}\right) \mu_{\mathbf{B}_{2}}\left(s_{2} t_{2}\right)}{\mu_{\mathbf{B}_{1}}\left(s_{1} t_{1}\right)+\mu_{\mathbf{B}_{2}}\left(s_{2} t_{2}\right)-\mu_{\mathbf{B}_{1}}\left(s_{1} t_{1}\right) \mu_{\mathbf{B}_{2}}\left(s_{2} t_{2}\right)}, \\
\left(\mathbf{D}_{v}\right)_{\mathbf{G}_{1} \bullet \mathbf{G}_{2}}\left(s_{1}, s_{2}\right)= & \sum_{\left(s_{1}, s_{2}\right)\left(t_{1}, t_{2}\right) \in \mathbf{E}_{1} \bullet \mathbf{E}_{2}}\left(v_{\mathbf{B}_{1}} \bullet v_{\mathbf{B}_{2}}\right)\left(\left(s_{1}, s_{2}\right)\left(t_{1}, t_{2}\right)\right) \\
= & \sum_{s_{1}=t_{1}, s_{2} t_{2} \in \mathbf{E}_{2}} \frac{v_{\mathbf{A}_{1}}\left(s_{1}\right)+v_{\mathbf{B}_{2}}\left(s_{2} t_{2}\right)-2 v_{\mathbf{A}_{1}}\left(s_{1}\right) v_{\mathbf{B}_{2}}\left(s_{2} t_{2}\right)}{1-v_{\mathbf{A}_{1}}\left(s_{1}\right) v_{\mathbf{B}_{2}}\left(s_{2} t_{2}\right)} \\
& +\sum_{s_{1} t_{1} \in \mathbf{E}_{1}, s_{2} t_{2} \in \mathbf{E}_{2}} \frac{v_{\mathbf{B}_{1}}\left(s_{1} t_{1}\right)+v_{\mathbf{B}_{2}}\left(s_{2} t_{2}\right)-2 v_{\mathbf{B}_{1}}\left(s_{1} t_{1}\right) v_{\mathbf{B}_{2}}\left(s_{2} t_{2}\right)}{1-v_{\mathbf{B}_{1}}\left(s_{1} t_{1}\right) v_{\mathbf{B}_{2}}\left(s_{2} t_{2}\right)} .
\end{aligned}
$$

Definition 10. Consider $\mathbf{G}_{1}=\left(\mathbf{A}_{1}, \mathbf{B}_{1}\right)$ and $\mathbf{G}_{2}=\left(\mathbf{A}_{2}, \mathbf{B}_{2}\right)$ to be two PDFEGs. Then, for any vertex $\left(s_{1}, s_{2}\right) \in \mathbf{V}_{1} \bullet \mathbf{V}_{2}$,

$$
\begin{aligned}
\left(\mathbf{T D}_{\mu}\right)_{\mathbf{G}_{1} \bullet \mathbf{G}_{2}}\left(s_{1}, s_{2}\right)= & \sum_{\left(s_{1}, s_{2}\right)\left(t_{1}, t_{2}\right) \in \mathbf{E}_{1} \bullet \mathbf{E}_{2}}\left(\mu_{\mathbf{B}_{1}} \bullet \mu_{\mathbf{B}_{2}}\right)\left(\left(s_{1}, s_{2}\right)\left(t_{1}, t_{2}\right)\right)+\left(\mu_{\mathbf{A}_{1}} \bullet \mu_{\mathbf{A}_{2}}\right)\left(s_{1}, s_{2}\right) \\
= & \sum_{s_{1}=t_{1}, s_{2} t_{2} \in \mathbf{E}_{2}} \frac{\mu_{\mathbf{A}_{1}}\left(s_{1}\right) \mu_{\mathbf{B}_{2}}\left(s_{2} t_{2}\right)}{\mu_{\mathbf{A}_{1}}\left(s_{1}\right)+\mu_{\mathbf{B}_{2}}\left(s_{2} t_{2}\right)-\mu_{\mathbf{A}_{1}}\left(s_{1}\right) \mu_{\mathbf{B}_{2}}\left(s_{2} t_{2}\right)} \\
& +\sum_{s_{1} t_{1} \in \mathbf{E}_{1}, s_{2} t_{2} \in \mathbf{E}_{2}} \frac{\mu_{\mathbf{B}_{1}}\left(s_{1} t_{1}\right)+\mu_{\mathbf{B}_{2}}\left(s_{2} t_{2}\right)-\mu_{\mathbf{B}_{1}}\left(s_{1} t_{1}\right) \mu_{\mathbf{B}_{2}}\left(s_{2} t_{2}\right)}{\mu_{\mathbf{B}_{1}}\left(s_{1}\right)} \\
& +\frac{\mu_{\mathbf{A}_{1}}\left(s_{1}\right) \mu_{\mathbf{A}_{2}}\left(s_{2}\right)}{\mu_{\mathbf{A}_{1}}\left(s_{1}\right)+\mu_{\mathbf{A}_{2}}\left(s_{2}\right)-\mu_{\mathbf{A}_{1}}\left(s_{1}\right) \mu_{\mathbf{A}_{2}}\left(s_{2}\right)} \\
\left(\mathbf{T D}_{v}\right)_{\mathbf{G}_{1} \bullet \mathbf{G}_{2}}\left(s_{1}, s_{2}\right)= & \sum_{\left(s_{1}, s_{2}\right)\left(t_{1}, t_{2}\right) \in \mathbf{E}_{1} \bullet \mathbf{E}_{2}}\left(v_{\mathbf{B}_{1}} \bullet v_{\mathbf{B}_{2}}\right)\left(\left(s_{1}, s_{2}\right)\left(t_{1}, t_{2}\right)\right)+\left(v_{\mathbf{A}_{1}} \bullet v_{\mathbf{A}_{2}}\right)\left(s_{1}, s_{2}\right) \\
= & \sum_{s_{1}=t_{1}, s_{2} t_{2} \in \mathbf{E}_{2}} \frac{v_{\mathbf{A}_{1}}\left(s_{1}\right)+v_{\mathbf{B}_{2}}\left(s_{2} t_{2}\right)-2 v_{\mathbf{A}_{1}}\left(s_{1}\right) v_{\mathbf{B}_{2}}\left(s_{2} t_{2}\right)}{1-v_{\mathbf{A}_{1}}\left(s_{1}\right) v_{\mathbf{B}_{2}}\left(s_{2} t_{2}\right)} \\
& +\sum_{s_{1} t_{1} \in \mathbf{E}_{1}, s_{2} t_{2} \in \mathbf{E}_{2}} \frac{v_{\mathbf{B}_{1}}\left(s_{1} t_{1}\right)+v_{\mathbf{B}_{2}}\left(s_{2} t_{2}\right)-2 v_{\mathbf{B}_{1}}\left(s_{1} t_{1}\right) v_{\mathbf{B}_{2}}\left(s_{2} t_{2}\right)}{1-v_{\mathbf{B}_{1}}\left(s_{1} t_{1}\right) v_{\mathbf{B}_{2}}\left(s_{2} t_{2}\right)} \\
& +\frac{v_{\mathbf{A}_{1}}\left(s_{1}\right)+v_{\mathbf{A}_{2}}\left(s_{2}\right)-2 v_{\mathbf{A}_{1}}\left(s_{1}\right) v_{\mathbf{A}_{2}}\left(s_{2}\right)}{1-v_{\mathbf{A}_{1}}\left(s_{1}\right) v_{\mathbf{A}_{2}}\left(s_{2}\right)} .
\end{aligned}
$$

Example 6. Consider PDFEGs $\mathbf{G}_{1}$ and $\mathbf{G}_{2}$ as in Example 3; their $\mathbf{G}_{1} \bullet \mathbf{G}_{2}$ is given in Figure 7 . Then, by Definition 9, we must have:

$$
\begin{aligned}
\left(\mathbf{D}_{\mu}\right)_{\mathbf{G}_{1}} \bullet \mathbf{G}_{2}\left(s_{2}, t_{1}\right) & =\quad\left(\mu_{\mathbf{B}_{1}} \bullet \mu_{\mathbf{B}_{2}}\right)\left(\left(s_{2}, t_{1}\right)\left(s_{2}, t_{2}\right)\right)+\left(\mu_{\mathbf{B}_{1}} \bullet \mu_{\mathbf{B}_{2}}\right)\left(\left(s_{2}, t_{1}\right)\left(s_{1}, t_{2}\right)\right) \\
& =\frac{\mu_{\mathbf{A}_{1}}\left(s_{2}\right) \mu_{\mathbf{B}_{2}}\left(t_{1} t_{2}\right)}{\mu_{\mathbf{A}_{1}}\left(s_{2}\right)+\mu_{\mathbf{B}_{2}}\left(t_{1} t_{2}\right)-\mu_{\mathbf{A}_{1}}\left(s_{2}\right) \mu_{\mathbf{B}_{2}}\left(t_{1} t_{2}\right)}+\frac{\mu_{\mathbf{B}_{1}}\left(s_{1} s_{2}\right) \mu_{\mathbf{B}_{2}}\left(t_{1} t_{2}\right)}{\mu_{\mathbf{B}_{1}}\left(s_{1} s_{2}\right)+\mu_{\mathbf{B}_{2}}\left(t_{1} t_{2}\right)-\mu_{\mathbf{B}_{1}}\left(s_{1} s_{2}\right) \mu_{\mathbf{B}_{2}}\left(t_{1} t_{2}\right)}=1.3, \\
\left(\mathbf{D}_{v}\right)_{\mathbf{G}_{1} \bullet \mathbf{G}_{2}}\left(s_{2}, t_{1}\right)= & \left(v_{\mathbf{B}_{1}} \bullet v_{\mathbf{B}_{2}}\right)\left(\left(s_{2}, t_{1}\right)\left(s_{2}, t_{2}\right)\right)+\left(v_{\mathbf{B}_{1}} \bullet v_{\mathbf{B}_{2}}\right)\left(\left(s_{2}, t_{1}\right)\left(s_{1}, t_{2}\right)\right) \\
& =\frac{v_{\mathbf{A}_{1}}\left(s_{2}\right)+v_{\mathbf{B}_{2}}\left(t_{1} t_{2}\right)-2 v_{\mathbf{A}_{1}}\left(s_{2}\right) \nu_{\mathbf{B}_{2}}\left(t_{1} t_{2}\right)}{1-v_{\mathbf{A}_{1}}\left(s_{2}\right) v_{\mathbf{B}_{2}}\left(t_{1} t_{2}\right)}+\frac{v_{\mathbf{B}_{1}}\left(s_{1} s_{2}\right)+v_{\mathbf{B}_{2}}\left(t_{1} t_{2}\right)-2 v_{\mathbf{B}_{1}}\left(s_{1} s_{2}\right) v_{\mathbf{B}_{2}}\left(t_{1} t_{2}\right)}{1-v_{\mathbf{B}_{1}}\left(s_{1} s_{2}\right) v_{\mathbf{B}_{2}}\left(t_{1} t_{2}\right)}=0 .
\end{aligned}
$$


Thus, $(\mathbf{D})_{\mathbf{G}_{1} \bullet \mathbf{G}_{2}}\left(s_{2}, t_{1}\right)=(1.3,0)$.

Further, by using Definition 10, we have:

$$
\begin{aligned}
\left(\mathbf{T D}_{\mu}\right)_{\mathbf{G}_{1} \bullet \mathbf{G}_{2}}\left(s_{2}, t_{1}\right) & =\left(\mathbf{D}_{\mu}\right)_{\mathbf{G}_{1} \bullet \mathbf{G}_{2}}\left(s_{2}, t_{1}\right)+\left(\mu_{\mathbf{A}_{1}} \bullet \mu_{\mathbf{A}_{2}}\right)\left(s_{2}, t_{1}\right) \\
& =1.3+\frac{\mu_{\mathbf{A}_{1}}\left(s_{2}\right) \mu_{\mathbf{A}_{2}}\left(t_{1}\right)}{\mu_{\mathbf{A}_{1}}\left(s_{2}\right)+\mu_{\mathbf{A}_{2}}\left(t_{1}\right)-\mu_{\mathbf{A}_{1}}\left(s_{2}\right) \mu_{\mathbf{A}_{2}}\left(t_{1}\right)}=2.3, \\
\left(\mathbf{T D}_{v}\right)_{\mathbf{G}_{1} \bullet \mathbf{G}_{2}}\left(s_{2}, t_{1}\right) & =\left(\mathbf{D}_{v}\right)_{\mathbf{G}_{1} \bullet \mathbf{G}_{2}}\left(s_{2}, t_{1}\right)+\left(v_{\mathbf{A}_{1}} \bullet v_{\mathbf{A}_{2}}\right)\left(s_{2}, t_{1}\right) \\
& =0+\frac{v_{\mathbf{A}_{1}}\left(s_{2}\right)+v_{\mathbf{A}_{2}}\left(t_{1}\right)-2 v_{\mathbf{A}_{1}}\left(s_{2}\right) v_{\mathbf{A}_{2}}\left(t_{1}\right)}{1-v_{\mathbf{A}_{1}}\left(s_{2}\right) v_{\mathbf{A}_{2}}\left(t_{1}\right)}=0 .
\end{aligned}
$$

Thus, $(\mathbf{T D})_{\mathbf{G}_{1} \bullet \mathbf{G}_{2}}\left(s_{2}, t_{1}\right)=(2.3,0)$.

Remark 3. The semi-strong product $\mathbf{G}_{1} \bullet \mathbf{G}_{2}$ of strong PDFGs $\mathbf{G}_{1}$ and $\mathbf{G}_{2}$ is not a PDFG. This is justified in the following example.

Consider strong PDFGs $\mathbf{G}_{1}$ and $\mathbf{G}_{2}$. Their $\mathbf{G}_{1} \bullet \mathbf{G}_{2}$ is displayed in Figure 8.

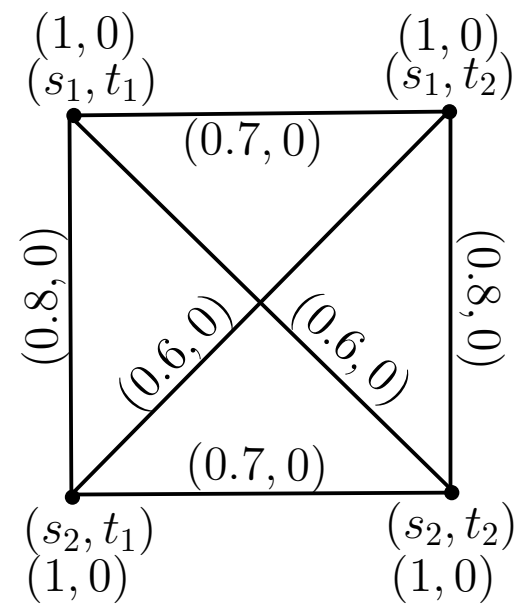

Figure 8. $\mathrm{G}_{1} \bullet \mathrm{G}_{2}$ is not a PDFG.

Since for the membership and non-membership grade of $\left(s_{1}, t_{1}\right)\left(s_{1}, t_{2}\right)$ :

$$
\begin{aligned}
\left(\mu_{\mathbf{B}_{1}} \bullet \mu_{\mathbf{B}_{2}}\right)\left(\left(s_{1}, t_{1}\right)\left(s_{1}, t_{2}\right)\right)=0.33 \not \leq 0.22= & \frac{\left(\mu_{\mathbf{A}_{1}} \bullet \mu_{\mathbf{A}_{2}}\right)\left(s_{1}, t_{1}\right)\left(\mu_{\mathbf{A}_{1}} \bullet \mu_{\mathbf{A}_{2}}\right)\left(s_{1}, t_{2}\right)}{\left(\mu_{\mathbf{A}_{1}} \bullet \mu_{\mathbf{A}_{2}}\right)\left(s_{1}, t_{1}\right)+\left(\mu_{\mathbf{A}_{1}} \bullet \mu_{\mathbf{A}_{2}}\right)\left(s_{1}, t_{2}\right)}, \\
& -\left(\mu_{\mathbf{A}_{1}} \bullet \mu_{\mathbf{A}_{2}}\right)\left(s_{1}, t_{1}\right)\left(\mu_{\mathbf{A}_{1}} \bullet \mu_{\mathbf{A}_{2}}\right)\left(s_{1}, t_{2}\right) \\
& \left(v_{\mathbf{A}_{1}} \bullet v_{\mathbf{A}_{2}}\right)\left(s_{1}, t_{1}\right)+\left(v_{\mathbf{A}_{1}} \bullet v_{\mathbf{A}_{2}}\right)\left(s_{1}, t_{2}\right) \\
\left(v_{\mathbf{B}_{1}} \bullet v_{\mathbf{B}_{2}}\right)\left(\left(s_{1}, t_{1}\right)\left(s_{1}, t_{2}\right)\right)=0.78 \leq 0.86= & \frac{-2\left(v_{\mathbf{A}_{1}} \bullet v_{\mathbf{A}_{2}}\right)\left(s_{1}, t_{1}\right)\left(v_{\mathbf{A}_{1}} \bullet v_{\mathbf{A}_{2}}\right)\left(s_{1}, t_{2}\right)}{1-\left(v_{\mathbf{A}_{1}} \bullet v_{\mathbf{A}_{2}}\right)\left(s_{1}, t_{1}\right)\left(v_{\mathbf{A}_{1}} \bullet v_{\mathbf{A}_{2}}\right)\left(s_{1}, t_{2}\right)} .
\end{aligned}
$$

Likewise, for the membership and non-membership grade of $\left(s_{1}, t_{1}\right)\left(s_{2}, t_{2}\right)$ :

$$
\begin{aligned}
\left(\mu_{\mathbf{B}_{1}} \bullet \mu_{\mathbf{B}_{2}}\right)\left(\left(s_{1}, t_{1}\right)\left(s_{2}, t_{2}\right)\right)=0.27=0.27= & \frac{\left(\mu_{\mathbf{A}_{1}} \bullet \mu_{\mathbf{A}_{2}}\right)\left(s_{1}, t_{1}\right)\left(\mu_{\mathbf{A}_{1}} \bullet \mu_{\mathbf{A}_{2}}\right)\left(s_{2}, t_{2}\right)}{\left(\mu_{\mathbf{A}_{1}} \bullet \mu_{\mathbf{A}_{2}}\right)\left(s_{1}, t_{1}\right)+\left(\mu_{\mathbf{A}_{1}} \bullet \mu_{\mathbf{A}_{2}}\right)\left(s_{2}, t_{2}\right)}, \\
& -\left(\mu_{\mathbf{A}_{1}} \bullet \mu_{\mathbf{A}_{2}}\right)\left(s_{1}, t_{1}\right)\left(\mu_{\mathbf{A}_{1}} \bullet \mu_{\mathbf{A}_{2}}\right)\left(s_{2}, t_{2}\right)
\end{aligned}
$$




$$
\begin{gathered}
\left(v_{\mathbf{A}_{1}} \bullet v_{\mathbf{A}_{2}}\right)\left(s_{1}, t_{1}\right)+\left(v_{\mathbf{A}_{1}} \bullet v_{\mathbf{A}_{2}}\right)\left(s_{2}, t_{2}\right) \\
\left(v_{\mathbf{B}_{1}} \bullet v_{\mathbf{B}_{2}}\right)\left(\left(s_{1}, t_{1}\right)\left(s_{2}, t_{2}\right)\right)=0.82=0.82=\frac{-2\left(v_{\mathbf{A}_{1}} \bullet v_{\mathbf{A}_{2}}\right)\left(s_{1}, t_{1}\right)\left(v_{\mathbf{A}_{1}} \bullet v_{\mathbf{A}_{2}}\right)\left(s_{2}, t_{2}\right)}{1-\left(v_{\mathbf{A}_{1}} \bullet v_{\mathbf{A}_{2}}\right)\left(s_{1}, t_{1}\right)\left(v_{\mathbf{A}_{1}} \bullet v_{\mathbf{A}_{2}}\right)\left(s_{2}, t_{2}\right)} .
\end{gathered}
$$

Hence, it is concluded that $\mathbf{G}_{1} \bullet \mathbf{G}_{2}$ is not a PDFG of underlying crisp graph $\mathbf{G}_{1}^{\prime} \bullet \mathbf{G}_{2}^{\prime}$.

Proposition 7. If $\mathbf{G}_{1}=\left(\mathbf{A}_{1}, \mathbf{B}_{1}\right)$ and $\mathbf{G}_{2}=\left(\mathbf{A}_{2}, \mathbf{B}_{2}\right)$ are strong PDFEGs of underlying crisp graphs $\mathbf{G}_{1}^{\prime}=$ $\left(\mathbf{V}_{1}, \mathbf{E}_{1}\right)$ and $\mathbf{G}_{2}^{\prime}=\left(\mathbf{V}_{2}, \mathbf{E}_{2}\right)$, respectively, then the semi-strong product $\mathbf{G}_{1} \bullet \mathbf{G}_{2}$ of $\mathbf{G}_{1}$ and $\mathbf{G}_{2}$ is also a strong PDFEG of $\mathbf{G}_{1}^{\prime} \bullet \mathbf{G}_{2}^{\prime}$.

\subsection{Strong Product of Pythagorean Dombi Fuzzy Graphs}

Definition 11. Let $\mathbf{A}_{j}$ and $\mathbf{B}_{j}$ be the Pythagorean fuzzy subsets of $\mathbf{V}_{j}$ and $\mathbf{E}_{j}(j=1,2)$, respectively. The strong product of PDFGs $\mathbf{G}_{1}=\left(\mathbf{A}_{1}, \mathbf{B}_{1}\right)$ and $\mathbf{G}_{2}=\left(\mathbf{A}_{2}, \mathbf{B}_{2}\right)$ of underlying crisp graphs $\mathbf{G}_{1}^{\prime}=\left(\mathbf{V}_{1}, \mathbf{E}_{1}\right)$ and $\mathbf{G}_{2}^{\prime}=\left(\mathbf{V}_{2}, \mathbf{E}_{2}\right)$, respectively, is represented by $\mathbf{G}_{1} \otimes \mathbf{G}_{2}=\left(\mathbf{A}_{1} \otimes \mathbf{A}_{2}, \mathbf{B}_{1} \otimes \mathbf{B}_{2}\right)$, defined as:

(i)

(i) $\left\{\begin{array}{l}\left(\mu_{\mathbf{A}_{1}} \otimes \mu_{\mathbf{A}_{2}}\right)\left(s_{1}, s_{2}\right)=\frac{\mu_{\mathbf{A}_{1}}\left(s_{1}\right) \mu_{\mathbf{A}_{2}}\left(s_{2}\right)}{\mu_{\mathbf{A}_{1}}\left(s_{1}\right)+\mu_{\mathbf{A}_{2}}\left(s_{2}\right)-\mu_{\mathbf{A}_{1}}\left(s_{1}\right) \mu_{\mathbf{A}_{2}}\left(s_{2}\right)} \\ \left(v_{\mathbf{A}_{1}} \otimes v_{\mathbf{A}_{2}}\right)\left(s_{1}, s_{2}\right)=\frac{v_{\mathbf{A}_{1}}\left(s_{1}\right)+v_{\mathbf{A}_{2}}\left(s_{2}\right)-2 v_{\mathbf{A}_{1}}\left(s_{1}\right) v_{\mathbf{A}_{2}}\left(s_{2}\right)}{1-v_{\mathbf{A}_{1}}\left(s_{1}\right) v_{\mathbf{A}_{2}}\left(s_{2}\right)}\end{array}\right.$ for all $\left(s_{1}, s_{2}\right) \in \mathbf{V}_{1} \otimes \mathbf{V}_{2}$,

(ii) $\left\{\begin{aligned}\left(\mu_{\mathbf{B}_{1}} \otimes \mu_{\mathbf{B}_{2}}\right)\left(\left(s, s_{2}\right)\left(s, t_{2}\right)\right) & =\frac{\mu_{\mathbf{A}_{1}}(s) \mu_{\mathbf{B}_{2}}\left(s_{2} t_{2}\right)}{\mu_{\mathbf{A}_{1}}(s)+\mu_{\mathbf{B}_{2}}\left(s_{2} t_{2}\right)-\mu_{\mathbf{A}_{1}}(s) \mu_{\mathbf{B}_{2}}\left(s_{2} t_{2}\right)} \\ \left(v_{\mathbf{B}_{1}} \otimes v_{\mathbf{B}_{2}}\right)\left(\left(s, s_{2}\right)\left(s, t_{2}\right)\right) & =\frac{v_{\mathbf{A}_{1}}(s)+v_{\mathbf{B}_{2}}\left(s_{2} t_{2}\right)-2 v_{\mathbf{A}_{1}}(s) v_{\mathbf{B}_{2}}\left(s_{2} t_{2}\right)}{1-v_{\mathbf{A}_{1}}(s) v_{\mathbf{B}_{2}}\left(s_{2} t_{2}\right)}\end{aligned}\right.$ for all $s \in \mathbf{V}_{1}$ and $s_{2} t_{2} \in \mathbf{E}_{2}$,

(iii) $\left\{\begin{aligned}\left(\mu_{\mathbf{B}_{1}} \otimes \mu_{\mathbf{B}_{2}}\right)\left(\left(s_{1}, s\right)\left(t_{1}, s\right)\right) & =\frac{\mu_{\mathbf{B}_{1}}\left(s_{1} t_{1}\right) \mu_{\mathbf{A}_{2}}(s)}{\mu_{\mathbf{B}_{1}}\left(s_{1} t_{1}\right)+\mu_{\mathbf{A}_{2}}(s)-\mu_{\mathbf{B}_{1}}\left(s_{1} t_{1}\right) \mu_{\mathbf{A}_{2}}(s)} \\ \left(v_{\mathbf{B}_{1}} \otimes v_{\mathbf{B}_{2}}\right)\left(\left(s_{1}, s\right)\left(t_{1}, s\right)\right) & =\frac{v_{\mathbf{B}_{1}}\left(s_{1} t_{1}\right)+v_{\mathbf{A}_{2}}(s)-2 v_{\mathbf{B}_{1}}\left(s_{1} t_{1}\right) v_{\mathbf{A}_{2}}(s)}{1-v_{\mathbf{B}_{1}}\left(s_{1} t_{1}\right) v_{\mathbf{A}_{2}}(s)}\end{aligned}\right.$ for all $s_{1} t_{1} \in \mathbf{E}_{1}$ and $s \in \mathbf{V}_{2}$.

(iv) $\left\{\begin{array}{l}\left(\mu_{\mathbf{B}_{1}} \otimes \mu_{\mathbf{B}_{2}}\right)\left(\left(s_{1}, s_{2}\right)\left(t_{1}, t_{2}\right)\right)=\frac{\mu_{\mathbf{B}_{1}}\left(s_{1} t_{1}\right) \mu_{\mathbf{B}_{2}}\left(s_{2} t_{2}\right)}{\mu_{\mathbf{B}_{1}}\left(s_{1} t_{1}\right)+\mu_{\mathbf{B}_{2}}\left(s_{2} t_{2}\right)-\mu_{\mathbf{B}_{1}}\left(s_{1} t_{1}\right) \mu_{\mathbf{B}_{2}}\left(s_{2} t_{2}\right)} \\ \left(v_{\mathbf{B}_{1}} \otimes v_{\mathbf{B}_{2}}\right)\left(\left(s_{1}, s_{2}\right)\left(t_{1}, t_{2}\right)\right)=\frac{v_{\mathbf{B}_{1}}\left(s_{1} t_{1}\right)+v_{\mathbf{B}_{2}}\left(s_{2} t_{2}\right)-2 v_{\mathbf{B}_{1}}\left(s_{1} t_{1}\right) \nu_{\mathbf{B}_{2}}\left(s_{2} t_{2}\right)}{1-v_{\mathbf{B}_{1}}\left(s_{1} t_{1}\right) v_{\mathbf{B}_{2}}\left(s_{2} t_{2}\right)}\end{array}\right.$ for all $s_{1} t_{1} \in \mathbf{E}_{1}$ and $s_{2} t_{2} \in \mathbf{E}_{2}$.

Proposition 8. If $\mathbf{G}_{1}=\left(\mathbf{A}_{1}, \mathbf{B}_{1}\right)$ and $\mathbf{G}_{2}=\left(\mathbf{A}_{2}, \mathbf{B}_{2}\right)$ are PDFEGs of underlying crisp graphs $\mathbf{G}_{1}^{\prime}=\left(\mathbf{V}_{1}, \mathbf{E}_{1}\right)$ and $\mathbf{G}_{2}^{\prime}=\left(\mathbf{V}_{2}, \mathbf{E}_{2}\right)$, respectively, then the strong product $\mathbf{G}_{1} \otimes \mathbf{G}_{2}$ of $\mathbf{G}_{1}$ and $\mathbf{G}_{2}$ is the PDFEG of $\mathbf{G}_{1}^{\prime} \otimes \mathbf{G}_{2}^{\prime}$.

Proof. This proposition can be easily proven in the same way as Proposition 1 and Proposition 4 has been proved.

Example 7. Consider PDFEGs $\mathbf{G}_{1}$ and $\mathbf{G}_{2}$ as in Example 3. Then, $\mathbf{G}_{1} \otimes \mathbf{G}_{2}$ is displayed in Figure 9. 

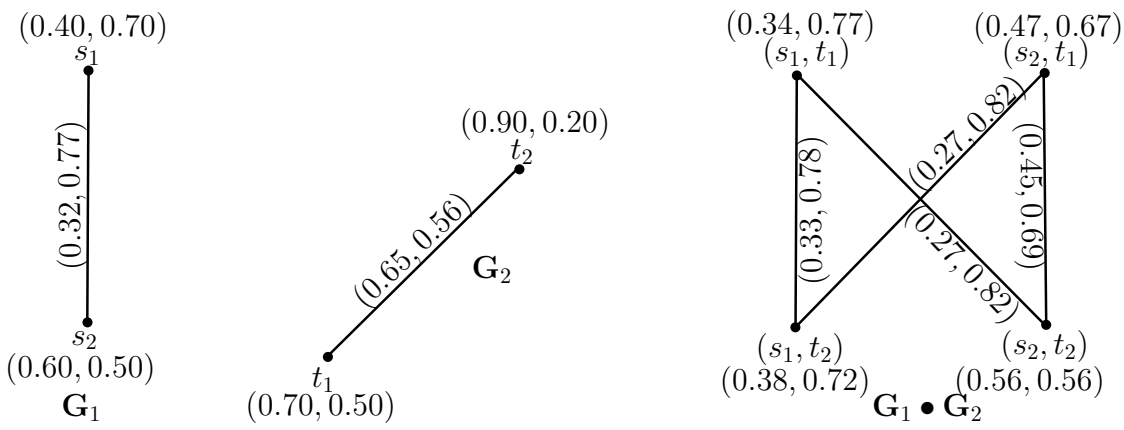

Figure 9. Strong product of two PDFEGs.

By routine computations, one can view from Figure 9 that $\mathbf{G}_{1} \otimes \mathbf{G}_{2}=\left(\mathbf{A}_{1} \otimes \mathbf{A}_{2}, \mathbf{B}_{1} \otimes \mathbf{B}_{2}\right)$ is a PDFEG of underlying crisp graph $\mathbf{G}_{1}^{\prime} \otimes \mathbf{G}_{2}^{\prime}=\left(\mathbf{V}_{1} \otimes \mathbf{V}_{2}, \mathbf{E}_{1} \otimes \mathbf{E}_{2}\right)$.

Definition 12. Consider $\mathbf{G}_{1}=\left(\mathbf{A}_{1}, \mathbf{B}_{1}\right)$ and $\mathbf{G}_{2}=\left(\mathbf{A}_{2}, \mathbf{B}_{2}\right)$ to be two PDFEGs. Then, for any vertex $\left(s_{1}, s_{2}\right) \in \mathbf{V}_{1} \otimes \mathbf{V}_{2}$,

$$
\begin{aligned}
& \left(\mathbf{D}_{\mu}\right)_{\mathbf{G}_{1} \otimes \mathbf{G}_{2}}\left(s_{1}, s_{2}\right)=\sum_{\left(s_{1}, s_{2}\right)\left(t_{1}, t_{2}\right) \in \mathbf{E}_{1} \otimes \mathbf{E}_{2}}\left(\mu_{\mathbf{B}_{1}} \otimes \mu_{\mathbf{B}_{2}}\right)\left(\left(s_{1}, s_{2}\right)\left(t_{1}, t_{2}\right)\right) \\
& =\sum_{s_{1}=t_{1}, s_{2} t_{2} \in \mathbf{E}_{2}} \frac{\mu_{\mathbf{A}_{1}}\left(s_{1}\right) \mu_{\mathbf{B}_{2}}\left(s_{2} t_{2}\right)}{\mu_{\mathbf{A}_{1}}\left(s_{1}\right)+\mu_{\mathbf{B}_{2}}\left(s_{2} t_{2}\right)-\mu_{\mathbf{A}_{1}}\left(s_{1}\right) \mu_{\mathbf{B}_{2}}\left(s_{2} t_{2}\right)} \\
& +\sum_{s_{1} t_{1} \in \mathbf{E}_{1}, s_{2}=t_{2}} \frac{\mu_{\mathbf{B}_{1}}\left(s_{1} t_{1}\right) \mu_{\mathbf{A}_{2}}\left(s_{2}\right)}{\mu_{\mathbf{B}_{1}}\left(s_{1} t_{1}\right)+\mu_{\mathbf{A}_{2}}\left(s_{2}\right)-\mu_{\mathbf{B}_{1}}\left(s_{1} t_{1}\right) \mu_{\mathbf{A}_{2}}\left(s_{2}\right)} \\
& +\sum_{s_{1} t_{1} \in \mathbf{E}_{1}, s_{2} t_{2} \in \mathbf{E}_{2}} \frac{\mu_{\mathbf{B}_{1}}\left(s_{1} t_{1}\right) \mu_{\mathbf{B}_{2}}\left(s_{2} t_{2}\right)}{\mu_{\mathbf{B}_{1}}\left(s_{1} t_{1}\right)+\mu_{\mathbf{B}_{2}}\left(s_{2} t_{2}\right)-\mu_{\mathbf{B}_{1}}\left(s_{1} t_{1}\right) \mu_{\mathbf{B}_{2}}\left(s_{2} t_{2}\right)}, \\
& \left(\mathbf{D}_{v}\right)_{\mathbf{G}_{1} \bowtie \mathbf{G}_{2}}\left(s_{1}, s_{2}\right)=\sum_{\left(s_{1}, s_{2}\right)\left(t_{1}, t_{2}\right) \in \mathbf{E}_{1} \bowtie \mathbf{E}_{2}}\left(v_{\mathbf{B}_{1}} \otimes v_{\mathbf{B}_{2}}\right)\left(\left(s_{1}, s_{2}\right)\left(t_{1}, t_{2}\right)\right) \\
& =\sum_{s_{1}=t_{1}, s_{2} t_{2} \in \mathbf{E}_{2}} \frac{v_{\mathbf{A}_{1}}\left(s_{1}\right)+v_{\mathbf{B}_{2}}\left(s_{2} t_{2}\right)-2 v_{\mathbf{A}_{1}}\left(s_{1}\right) v_{\mathbf{B}_{2}}\left(s_{2} t_{2}\right)}{1-v_{\mathbf{A}_{1}}\left(s_{1}\right) v_{\mathbf{B}_{2}}\left(s_{2} t_{2}\right)} \\
& +\sum_{s_{1} t_{1} \in \mathbf{E}_{1}, s_{2}=t_{2}} \frac{v_{\mathbf{B}_{1}}\left(s_{1} t_{1}\right)+v_{\mathbf{A}_{2}}\left(s_{2}\right)-2 v_{\mathbf{B}_{1}}\left(s_{1} t_{1}\right) v_{\mathbf{A}_{2}}\left(s_{2}\right)}{1-v_{\mathbf{B}_{1}}\left(s_{1} t_{1}\right) v_{\mathbf{A}_{2}}\left(s_{2}\right)} \\
& +\sum_{s_{1} t_{1} \in \mathbf{E}_{1}, s_{2} t_{2} \in \mathbf{E}_{2}} \frac{v_{\mathbf{B}_{1}}\left(s_{1} t_{1}\right)+v_{\mathbf{B}_{2}}\left(s_{2} t_{2}\right)-2 v_{\mathbf{B}_{1}}\left(s_{1} t_{1}\right) v_{\mathbf{B}_{2}}\left(s_{2} t_{2}\right)}{1-v_{\mathbf{B}_{1}}\left(s_{1} t_{1}\right) v_{\mathbf{B}_{2}}\left(s_{2} t_{2}\right)} .
\end{aligned}
$$


Definition 13. Consider $\mathbf{G}_{1}=\left(\mathbf{A}_{1}, \mathbf{B}_{1}\right)$ and $\mathbf{G}_{2}=\left(\mathbf{A}_{2}, \mathbf{B}_{2}\right)$ to be two PDFEGs. Then, for any vertex $\left(s_{1}, s_{2}\right) \in \mathbf{V}_{1} \otimes \mathbf{V}_{2}$,

$$
\begin{aligned}
& \left(\mathbf{T D}_{\mu}\right)_{\mathbf{G}_{1} \otimes \mathbf{G}_{2}}\left(s_{1}, s_{2}\right)=\sum_{\left(s_{1}, s_{2}\right)\left(t_{1}, t_{2}\right) \in \mathbf{E}_{1} \otimes \mathbf{E}_{2}}\left(\mu_{\mathbf{B}_{1}} \otimes \mu_{\mathbf{B}_{2}}\right)\left(\left(s_{1}, s_{2}\right)\left(t_{1}, t_{2}\right)\right)+\left(\mu_{\mathbf{A}_{1}} \otimes \mu_{\mathbf{A}_{2}}\right)\left(s_{1}, s_{2}\right) \\
& =\sum_{s_{1}=t_{1}, s_{2} t_{2} \in \mathbf{E}_{2}} \frac{\mu_{\mathbf{A}_{1}}\left(s_{1}\right) \mu_{\mathbf{B}_{2}}\left(s_{2} t_{2}\right)}{\mu_{\mathbf{A}_{1}}\left(s_{1}\right)+\mu_{\mathbf{B}_{2}}\left(s_{2} t_{2}\right)-\mu_{\mathbf{A}_{1}}\left(s_{1}\right) \mu_{\mathbf{B}_{2}}\left(s_{2} t_{2}\right)} \\
& +\sum_{s_{1} t_{1} \in \mathbf{E}_{1}, s_{2}=t_{2}} \frac{\mu_{\mathbf{B}_{1}}\left(s_{1} t_{1}\right) \mu_{\mathbf{A}_{2}}\left(s_{2}\right)}{\mu_{\mathbf{B}_{1}}\left(s_{1} t_{1}\right)+\mu_{\mathbf{A}_{2}}\left(s_{2}\right)-\mu_{\mathbf{B}_{1}}\left(s_{1} t_{1}\right) \mu_{\mathbf{A}_{2}}\left(s_{2}\right)} \\
& +\sum_{s_{1} t_{1} \in \mathbf{E}_{1}, s_{2} t_{2} \in \mathbf{E}_{2}} \frac{\mu_{\mathbf{B}_{1}}\left(s_{1} t_{1}\right) \mu_{\mathbf{B}_{2}}\left(s_{2} t_{2}\right)}{\mu_{\mathbf{B}_{1}}\left(s_{1} t_{1}\right)+\mu_{\mathbf{B}_{2}}\left(s_{2} t_{2}\right)-\mu_{\mathbf{B}_{1}}\left(s_{1} t_{1}\right) \mu_{\mathbf{B}_{2}}\left(s_{2} t_{2}\right)} \\
& +\frac{\mu_{\mathbf{A}_{1}}\left(s_{1}\right) \mu_{\mathbf{A}_{2}}\left(s_{2}\right)}{\mu_{\mathbf{A}_{1}}\left(s_{1}\right)+\mu_{\mathbf{A}_{2}}\left(s_{2}\right)-\mu_{\mathbf{A}_{1}}\left(s_{1}\right) \mu_{\mathbf{A}_{2}}\left(s_{2}\right)}, \\
& \left(\mathbf{T D}_{v}\right)_{\mathbf{G}_{1} \bowtie \mathbf{G}_{2}}\left(s_{1}, s_{2}\right)=\sum_{\left(s_{1}, s_{2}\right)\left(t_{1}, t_{2}\right) \in \mathbf{E}_{1} \bowtie \mathbf{E}_{2}}\left(v_{\mathbf{B}_{1}} \otimes v_{\mathbf{B}_{2}}\right)\left(\left(s_{1}, s_{2}\right)\left(t_{1}, t_{2}\right)\right)+\left(v_{\mathbf{A}_{1}} \otimes v_{\mathbf{A}_{2}}\right)\left(s_{1}, s_{2}\right) \\
& =\sum_{s_{1}=t_{1}, s_{2} t_{2} \in \mathbf{E}_{2}} \frac{v_{\mathbf{A}_{1}}\left(s_{1}\right)+v_{\mathbf{B}_{2}}\left(s_{2} t_{2}\right)-2 v_{\mathbf{A}_{1}}\left(s_{1}\right) v_{\mathbf{B}_{2}}\left(s_{2} t_{2}\right)}{1-v_{\mathbf{A}_{1}}\left(s_{1}\right) v_{\mathbf{B}_{2}}\left(s_{2} t_{2}\right)} \\
& +\sum_{s_{1} t_{1} \in \mathbf{E}_{1}, s_{2}=t_{2}} \frac{v_{\mathbf{B}_{1}}\left(s_{1} t_{1}\right)+v_{\mathbf{A}_{2}}\left(s_{2}\right)-2 v_{\mathbf{B}_{1}}\left(s_{1} t_{1}\right) v_{\mathbf{A}_{2}}\left(s_{2}\right)}{1-v_{\mathbf{B}_{1}}\left(s_{1} t_{1}\right) v_{\mathbf{A}_{2}}\left(s_{2}\right)} \\
& +\sum_{s_{1} t_{1} \in \mathbf{E}_{1}, s_{2} t_{2} \in \mathbf{E}_{2}} \frac{v_{\mathbf{B}_{1}}\left(s_{1} t_{1}\right)+v_{\mathbf{B}_{2}}\left(s_{2} t_{2}\right)-2 v_{\mathbf{B}_{1}}\left(s_{1} t_{1}\right) v_{\mathbf{B}_{2}}\left(s_{2} t_{2}\right)}{1-v_{\mathbf{B}_{1}}\left(s_{1} t_{1}\right) v_{\mathbf{B}_{2}}\left(s_{2} t_{2}\right)} \\
& +\frac{v_{\mathbf{A}_{1}}\left(s_{1}\right)+v_{\mathbf{A}_{2}}\left(s_{2}\right)-2 v_{\mathbf{A}_{1}}\left(s_{1}\right) v_{\mathbf{A}_{2}}\left(s_{2}\right)}{1-v_{\mathbf{A}_{1}}\left(s_{1}\right) v_{\mathbf{A}_{2}}\left(s_{2}\right)} .
\end{aligned}
$$

Example 8. Consider PDFEGs $\mathbf{G}_{1}$ and $\mathbf{G}_{2}$ as in Example 3; their $\mathbf{G}_{1} \otimes \mathbf{G}_{2}$ is given in Figure 9. Then, by Definition 12, we must have:

$$
\begin{aligned}
& \left(\mathbf{D}_{\mu}\right)_{\mathbf{G}_{1} \otimes \mathbf{G}_{2}}\left(s_{1}, t_{1}\right)=\begin{array}{l}
\left(\mu_{\mathbf{B}_{1}} \otimes \mu_{\mathbf{B}_{2}}\right)\left(\left(s_{1}, t_{1}\right)\left(s_{1}, t_{2}\right)\right)+\left(\mu_{\mathbf{B}_{1}} \otimes \mu_{\mathbf{B}_{2}}\right)\left(\left(s_{1}, t_{1}\right)\left(s_{2}, t_{1}\right)\right)+\left(\mu_{\mathbf{B}_{1}} \otimes \mu_{\mathbf{B}_{2}}\right)\left(\left(s_{1}, t_{1}\right)\right. \\
\left.\left(s_{2}, t_{2}\right)\right)
\end{array} \\
& =\frac{\mu_{\mathbf{A}_{1}}\left(s_{1}\right) \mu_{\mathbf{B}_{2}}\left(t_{1} t_{2}\right)}{\mu_{\mathbf{A}_{1}}\left(s_{1}\right)+\mu_{\mathbf{B}_{2}}\left(t_{1} t_{2}\right)-\mu_{\mathbf{A}_{1}}\left(s_{1}\right) \mu_{\mathbf{B}_{2}}\left(t_{1} t_{2}\right)}+\frac{\mu_{\mathbf{B}_{1}}\left(s_{1} s_{2}\right) \mu_{\mathbf{A}_{2}}\left(t_{1}\right)}{\mu_{\mathbf{B}_{1}}\left(s_{1} s_{2}\right)+\mu_{\mathbf{A}_{2}}\left(t_{1}\right)-\mu_{\mathbf{B}_{1}}\left(s_{1} s_{2}\right) \mu_{\mathbf{A}_{2}}\left(t_{1}\right)} \\
& +\frac{\mu_{\mathbf{B}_{1}}\left(s_{1} s_{2}\right) \mu_{\mathbf{B}_{2}}\left(t_{1} t_{2}\right)}{\mu_{\mathbf{B}_{1}}\left(s_{1} s_{2}\right)+\mu_{\mathbf{B}_{2}}\left(t_{1} t_{2}\right)-\mu_{\mathbf{B}_{1}}\left(s_{1} s_{2}\right) \mu_{\mathbf{B}_{2}}\left(t_{1} t_{2}\right)}=0.7+0.8+0.6=2.1, \\
& \left(\mathbf{D}_{v}\right)_{\mathbf{G}_{1} \bowtie \mathbf{G}_{2}}\left(s_{1}, t_{1}\right)=\begin{array}{l}
\left(v_{\mathbf{B}_{1}} \otimes v_{\mathbf{B}_{2}}\right)\left(\left(s_{1}, t_{1}\right)\left(s_{1}, t_{2}\right)\right)+\left(v_{\mathbf{B}_{1}} \otimes v_{\mathbf{B}_{2}}\right)\left(\left(s_{1}, t_{1}\right)\left(s_{2}, t_{1}\right)\right)+\left(v_{\mathbf{B}_{1}} \otimes v_{\mathbf{B}_{2}}\right)\left(\left(s_{1}, t_{1}\right)\right. \\
\left.\left(s_{2}, t_{2}\right)\right)
\end{array} \\
& =\frac{v_{\mathbf{A}_{1}}\left(s_{1}\right)+v_{\mathbf{B}_{2}}\left(t_{1} t_{2}\right)-2 v_{\mathbf{A}_{1}}\left(s_{1}\right) v_{\mathbf{B}_{2}}\left(t_{1} t_{2}\right)}{1-v_{\mathbf{A}_{1}}\left(s_{1}\right) v_{\mathbf{B}_{2}}\left(t_{1} t_{2}\right)}+\frac{v_{\mathbf{B}_{1}}\left(s_{1} s_{2}\right)+v_{\mathbf{A}_{2}}\left(t_{1}\right)-2 v_{\mathbf{B}_{1}}\left(s_{1} s_{2}\right) v_{\mathbf{A}_{2}}\left(t_{1}\right)}{1-v_{\mathbf{B}_{1}}\left(s_{1} s_{2}\right) v_{\mathbf{A}_{2}}\left(t_{1}\right)} \\
& +\frac{v_{\mathbf{B}_{1}}\left(s_{1} s_{2}\right)+v_{\mathbf{B}_{2}}\left(t_{1} t_{2}\right)-2 v_{\mathbf{B}_{1}}\left(s_{1} s_{2}\right) v_{\mathbf{B}_{2}}\left(t_{1} t_{2}\right)}{1-v_{\mathbf{B}_{1}}\left(s_{1} s_{2}\right) v_{\mathbf{B}_{2}}\left(t_{1} t_{2}\right)}=0+0+0=0 .
\end{aligned}
$$


Thus, $(\mathbf{D})_{\mathbf{G}_{1} \bowtie \mathbf{G}_{2}}\left(s_{1}, t_{1}\right)=(2.1,0)$.

Further, by using Definition 13, we have:

$$
\begin{aligned}
\left(\mathbf{T D}_{\mu}\right)_{\mathbf{G}_{1} \otimes \mathbf{G}_{2}}\left(s_{1}, t_{1}\right)= & \left(\mathbf{D}_{\mu}\right)_{\mathbf{G}_{1} \bowtie \mathbf{G}_{2}}\left(s_{1}, t_{1}\right)+\left(\mu_{\mathbf{A}_{1}} \otimes \mu_{\mathbf{A}_{2}}\right)\left(s_{1}, t_{1}\right) \\
= & 2.1+\frac{\mu_{\mathbf{A}_{1}}\left(s_{1}\right) \mu_{\mathbf{A}_{2}}\left(t_{1}\right)}{\mu_{\mathbf{A}_{1}}\left(s_{1}\right)+\mu_{\mathbf{A}_{2}}\left(t_{1}\right)-\mu_{\mathbf{A}_{1}}\left(s_{1}\right) \mu_{\mathbf{A}_{2}}\left(t_{1}\right)}=3.1, \\
\left(\mathbf{T D}_{v}\right)_{\mathbf{G}_{1} \bowtie \mathbf{G}_{2}}\left(s_{1}, t_{1}\right)= & \left(\mathbf{D}_{v}\right)_{\mathbf{G}_{1} \bowtie \mathbf{G}_{2}}\left(s_{1}, t_{1}\right)+\left(v_{\mathbf{A}_{1}} \otimes v_{\mathbf{A}_{2}}\right)\left(s_{1}, t_{1}\right) \\
& =0+\frac{v_{\mathbf{A}_{1}}\left(s_{1}\right)+v_{\mathbf{A}_{2}}\left(t_{1}\right)-2 v_{\mathbf{A}_{1}}\left(s_{1}\right) v_{\mathbf{A}_{2}}\left(t_{1}\right)}{1-v_{\mathbf{A}_{1}}\left(s_{1}\right) v_{\mathbf{A}_{2}}\left(t_{1}\right)}=0 .
\end{aligned}
$$

Thus, $(\mathbf{T D})_{\mathbf{G}_{1} \otimes \mathbf{G}_{2}}\left(s_{1}, t_{1}\right)=(3.1,0)$.

Remark 4. The strong product $\mathbf{G}_{1} \otimes \mathbf{G}_{2}$ of two strong PDFGs $\mathbf{G}_{1}$ and $\mathbf{G}_{2}$ is not a PDFG. It is justified in the following example.

Consider two strong PDFGs $\mathbf{G}_{1}$ and $\mathbf{G}_{2}$. Their $\mathbf{G}_{1} \otimes \mathbf{G}_{2}$ is displayed in Figure 10.
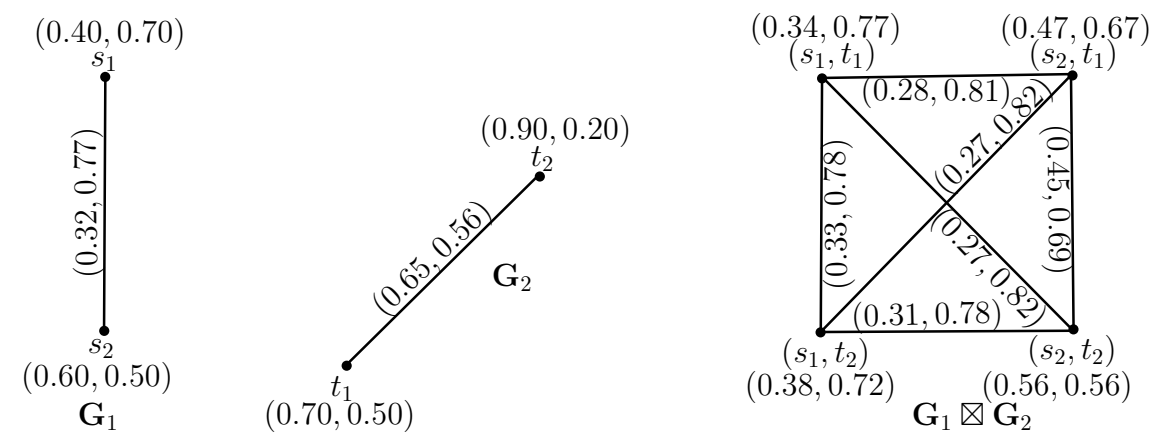

Figure 10. $G_{1} \otimes G_{2}$ is not a PDFG.

Since for the membership and non-membership grade of $\left(s_{1}, t_{1}\right)\left(s_{1}, t_{2}\right)$ :

$$
\begin{aligned}
& \left(\mu_{\mathbf{B}_{1}} \otimes \mu_{\mathbf{B}_{2}}\right)\left(\left(s_{1}, t_{1}\right)\left(s_{1}, t_{2}\right)\right)=0.33 \not \leq 0.22=\frac{\left(\mu_{\mathbf{A}_{1}} \otimes \mu_{\mathbf{A}_{2}}\right)\left(s_{1}, t_{1}\right)\left(\mu_{\mathbf{A}_{1}} \otimes \mu_{\mathbf{A}_{2}}\right)\left(s_{1}, t_{2}\right)}{\left(\mu_{\mathbf{A}_{1}} \otimes \mu_{\mathbf{A}_{2}}\right)\left(s_{1}, t_{1}\right)+\left(\mu_{\mathbf{A}_{1}} \otimes \mu_{\mathbf{A}_{2}}\right)\left(s_{1}, t_{2}\right)}, \\
& -\left(\mu_{\mathbf{A}_{1}} \otimes \mu_{\mathbf{A}_{2}}\right)\left(s_{1}, t_{1}\right)\left(\mu_{\mathbf{A}_{1}} \otimes \mu_{\mathbf{A}_{2}}\right)\left(s_{1}, t_{2}\right) \\
& \left(v_{\mathbf{A}_{1}} \otimes v_{\mathbf{A}_{2}}\right)\left(s_{1}, t_{1}\right)+\left(v_{\mathbf{A}_{1}} \otimes v_{\mathbf{A}_{2}}\right)\left(s_{1}, t_{2}\right) \\
& \left(v_{\mathbf{B}_{1}} \otimes v_{\mathbf{B}_{2}}\right)\left(\left(s_{1}, t_{1}\right)\left(s_{1}, t_{2}\right)\right)=0.78 \leq 0.86=\frac{-2\left(v_{\mathbf{A}_{1}} \otimes v_{\mathbf{A}_{2}}\right)\left(s_{1}, t_{1}\right)\left(v_{\mathbf{A}_{1}} \otimes v_{\mathbf{A}_{2}}\right)\left(s_{1}, t_{2}\right)}{1-\left(v_{\mathbf{A}_{1}} \otimes v_{\mathbf{A}_{2}}\right)\left(s_{1}, t_{1}\right)\left(v_{\mathbf{A}_{1}} \otimes v_{\mathbf{A}_{2}}\right)\left(s_{1}, t_{2}\right)} .
\end{aligned}
$$

Likewise, for the membership and non-membership grade of $\left(s_{1}, t_{1}\right)\left(s_{2}, t_{1}\right)$ :

$$
\begin{aligned}
\left(\mu_{\mathbf{B}_{1}} \otimes \mu_{\mathbf{B}_{2}}\right)\left(\left(s_{1}, t_{1}\right)\left(s_{2}, t_{1}\right)\right)=0.28 \not \leq 0.25= & \frac{\left(\mu_{\mathbf{A}_{1}} \otimes \mu_{\mathbf{A}_{2}}\right)\left(s_{1}, t_{1}\right)\left(\mu_{\mathbf{A}_{1}} \otimes \mu_{\mathbf{A}_{2}}\right)\left(s_{2}, t_{1}\right)}{\left(\mu_{\mathbf{A}_{1}} \otimes \mu_{\mathbf{A}_{2}}\right)\left(s_{1}, t_{1}\right)+\left(\mu_{\mathbf{A}_{1}} \otimes \mu_{\mathbf{A}_{2}}\right)\left(s_{2}, t_{1}\right)}, \\
& -\left(\mu_{\mathbf{A}_{1}} \otimes \mu_{\mathbf{A}_{2}}\right)\left(s_{1}, t_{1}\right)\left(\mu_{\mathbf{A}_{1}} \otimes \mu_{\mathbf{A}_{2}}\right)\left(s_{2}, t_{1}\right)
\end{aligned}
$$




$$
\begin{gathered}
\left(v_{\mathbf{A}_{1}} \otimes v_{\mathbf{A}_{2}}\right)\left(s_{1}, t_{1}\right)+\left(v_{\mathbf{A}_{1}} \otimes v_{\mathbf{A}_{2}}\right)\left(s_{2}, t_{1}\right) \\
\left(v_{\mathbf{B}_{1}} \otimes v_{\mathbf{B}_{2}}\right)\left(\left(s_{1}, t_{1}\right)\left(s_{2}, t_{1}\right)\right)=0.81 \leq 0.84=\frac{-2\left(v_{\mathbf{A}_{1}} \otimes v_{\mathbf{A}_{2}}\right)\left(s_{1}, t_{1}\right)\left(v_{\mathbf{A}_{1}} \otimes v_{\mathbf{A}_{2}}\right)\left(s_{2}, t_{1}\right)}{1-\left(v_{\mathbf{A}_{1}} \otimes v_{\mathbf{A}_{2}}\right)\left(s_{1}, t_{1}\right)\left(v_{\mathbf{A}_{1}} \otimes v_{\mathbf{A}_{2}}\right)\left(s_{2}, t_{1}\right)} .
\end{gathered}
$$

Further, for the membership and non-membership grade of $\left(s_{1}, t_{1}\right)\left(s_{2}, t_{2}\right)$ :

$$
\begin{aligned}
& \left(\mu_{\mathbf{B}_{1}} \otimes \mu_{\mathbf{B}_{2}}\right)\left(\left(s_{1}, t_{1}\right)\left(s_{2}, t_{2}\right)\right)=0.27=0.27=\frac{\left(\mu_{\mathbf{A}_{1}} \otimes \mu_{\mathbf{A}_{2}}\right)\left(s_{1}, t_{1}\right)\left(\mu_{\mathbf{A}_{1}} \otimes \mu_{\mathbf{A}_{2}}\right)\left(s_{2}, t_{2}\right)}{\left(\mu_{\mathbf{A}_{1}} \otimes \mu_{\mathbf{A}_{2}}\right)\left(s_{1}, t_{1}\right)+\left(\mu_{\mathbf{A}_{1}} \otimes \mu_{\mathbf{A}_{2}}\right)\left(s_{2}, t_{2}\right)}, \\
& -\left(\mu_{\mathbf{A}_{1}} \otimes \mu_{\mathbf{A}_{2}}\right)\left(s_{1}, t_{1}\right)\left(\mu_{\mathbf{A}_{1}} \otimes \mu_{\mathbf{A}_{2}}\right)\left(s_{2}, t_{2}\right) \\
& \left(v_{\mathbf{A}_{1}} \otimes v_{\mathbf{A}_{2}}\right)\left(s_{1}, t_{1}\right)+\left(v_{\mathbf{A}_{1}} \otimes v_{\mathbf{A}_{2}}\right)\left(s_{2}, t_{2}\right) \\
& \left(v_{\mathbf{B}_{1}} \otimes v_{\mathbf{B}_{2}}\right)\left(\left(s_{1}, t_{1}\right)\left(s_{2}, t_{2}\right)\right)=0.82=0.82=\frac{-2\left(v_{\mathbf{A}_{1}} \otimes v_{\mathbf{A}_{2}}\right)\left(s_{1}, t_{1}\right)\left(v_{\mathbf{A}_{1}} \otimes v_{\mathbf{A}_{2}}\right)\left(s_{2}, t_{2}\right)}{1-\left(v_{\mathbf{A}_{1}} \otimes v_{\mathbf{A}_{2}}\right)\left(s_{1}, t_{1}\right)\left(v_{\mathbf{A}_{1}} \otimes v_{\mathbf{A}_{2}}\right)\left(s_{2}, t_{2}\right)} .
\end{aligned}
$$

Hence, it is concluded that $\mathbf{G}_{1} \otimes \mathbf{G}_{2}$ is not a PDFG of underlying crisp graph $\mathbf{G}_{1}^{\prime} \otimes \mathbf{G}_{2}^{\prime}$.

Proposition 9. If $\mathbf{G}_{1}=\left(\mathbf{A}_{1}, \mathbf{B}_{1}\right)$ and $\mathbf{G}_{2}=\left(\mathbf{A}_{2}, \mathbf{B}_{2}\right)$ are strong PDFEGs of underlying crisp graphs $\mathbf{G}_{1}^{\prime}=$ $\left(\mathbf{V}_{1}, \mathbf{E}_{1}\right)$ and $\mathbf{G}_{2}^{\prime}=\left(\mathbf{V}_{2}, \mathbf{E}_{2}\right)$, respectively, then the strong product $\mathbf{G}_{1} \otimes \mathbf{G}_{2}$ of $\mathbf{G}_{1}$ and $\mathbf{G}_{2}$ is also a strong PDFEG of $\mathbf{G}_{1}^{\prime} \otimes \mathbf{G}_{2}^{\prime}$.

\subsection{Composition of Pythagorean Dombi Fuzzy Graphs}

Definition 14. Let $\mathbf{A}_{j}$ and $\mathbf{B}_{j}$ be the Pythagorean fuzzy subsets of $\mathbf{V}_{j}$ and $\mathbf{E}_{j}(j=1,2)$, respectively. The composition of PDFGs $\mathbf{G}_{1}=\left(\mathbf{A}_{1}, \mathbf{B}_{1}\right)$ and $\mathbf{G}_{2}=\left(\mathbf{A}_{2}, \mathbf{B}_{2}\right)$ of underlying crisp graphs $\mathbf{G}_{1}^{\prime}=\left(\mathbf{V}_{1}, \mathbf{E}_{1}\right)$ and $\mathbf{G}_{2}^{\prime}=\left(\mathbf{V}_{2}, \mathbf{E}_{2}\right)$, respectively, is represented by $\mathbf{G}_{1} \circ \mathbf{G}_{2}=\left(\mathbf{A}_{1} \circ \mathbf{A}_{2}, \mathbf{B}_{1} \circ \mathbf{B}_{2}\right)$, defined as:

(i)

$$
\begin{aligned}
& \left\{\begin{array}{l}
\left(\mu_{\mathbf{A}_{1}} \circ \mu_{\mathbf{A}_{2}}\right)\left(s_{1}, s_{2}\right)=\frac{\mu_{\mathbf{A}_{1}}\left(s_{1}\right) \mu_{\mathbf{A}_{2}}\left(s_{2}\right)}{\mu_{\mathbf{A}_{1}}\left(s_{1}\right)+\mu_{\mathbf{A}_{2}}\left(s_{2}\right)-\mu_{\mathbf{A}_{1}}\left(s_{1}\right) \mu_{\mathbf{A}_{2}}\left(s_{2}\right)} \\
\left(v_{\mathbf{A}_{1}} \circ v_{\mathbf{A}_{2}}\right)\left(s_{1}, s_{2}\right)=\frac{v_{\mathbf{A}_{1}}\left(s_{1}\right)+v_{\mathbf{A}_{2}}\left(s_{2}\right)-2 v_{\mathbf{A}_{1}}\left(s_{1}\right) v_{\mathbf{A}_{2}}\left(s_{2}\right)}{1-v_{\mathbf{A}_{1}}\left(s_{1}\right) v_{\mathbf{A}_{2}}\left(s_{2}\right)}
\end{array}\right. \\
& \text { for all }\left(s_{1}, s_{2}\right) \in \mathbf{V}_{1} \times \mathbf{V}_{2},
\end{aligned}
$$

(ii) $\left\{\begin{array}{l}\left(\mu_{\mathbf{B}_{1}} \circ \mu_{\mathbf{B}_{2}}\right)\left(\left(s, s_{2}\right)\left(s, t_{2}\right)\right)=\frac{\mu_{\mathbf{A}_{1}}(s) \mu_{\mathbf{B}_{2}}\left(s_{2} t_{2}\right)}{\mu_{\mathbf{A}_{1}}(s)+\mu_{\mathbf{B}_{2}}\left(s_{2} t_{2}\right)-\mu_{\mathbf{A}_{1}}(s) \mu_{\mathbf{B}_{2}}\left(s_{2} t_{2}\right)} \\ \left(v_{\mathbf{B}_{1}} \circ v_{\mathbf{B}_{2}}\right)\left(\left(s, s_{2}\right)\left(s, t_{2}\right)\right)=\frac{v_{\mathbf{A}_{1}}(s)+v_{\mathbf{B}_{2}}\left(s_{2} t_{2}\right)-2 v_{\mathbf{A}_{1}}(s) \nu_{\mathbf{B}_{2}}\left(s_{2} t_{2}\right)}{1-v_{\mathbf{A}_{1}}(s) v_{\mathbf{B}_{2}}\left(s_{2} t_{2}\right)}\end{array}\right.$ for all $s \in \mathbf{V}_{1}$ and $s_{2} t_{2} \in \mathbf{E}_{2}$,

(iii) $\left\{\begin{array}{l}\left(\mu_{\mathbf{B}_{1}} \circ \mu_{\mathbf{B}_{2}}\right)\left(\left(s_{1}, s\right)\left(t_{1}, s\right)\right)=\frac{\mu_{\mathbf{B}_{1}}\left(s_{1} t_{1}\right) \mu_{\mathbf{A}_{2}}(s)}{\mu_{\mathbf{B}_{1}}\left(s_{1} t_{1}\right)+\mu_{\mathbf{A}_{2}}(s)-\mu_{\mathbf{B}_{1}}\left(s_{1} t_{1}\right) \mu_{\mathbf{A}_{2}}(s)} \\ \left(v_{\mathbf{B}_{1}} \circ v_{\mathbf{B}_{2}}\right)\left(\left(s_{1}, s\right)\left(t_{1}, s\right)\right)=\frac{v_{\mathbf{B}_{1}}\left(s_{1} t_{1}\right)+v_{\mathbf{A}_{2}}(s)-2 v_{\mathbf{B}_{1}}\left(s_{1} t_{1}\right) v_{\mathbf{A}_{2}}(s)}{1-v_{\mathbf{B}_{1}}\left(s_{1} t_{1}\right) \nu_{\mathbf{A}_{2}}(s)}\end{array}\right.$ for all $s_{1} t_{1} \in \mathbf{E}_{1}$ and $s \in \mathbf{V}_{2}$.

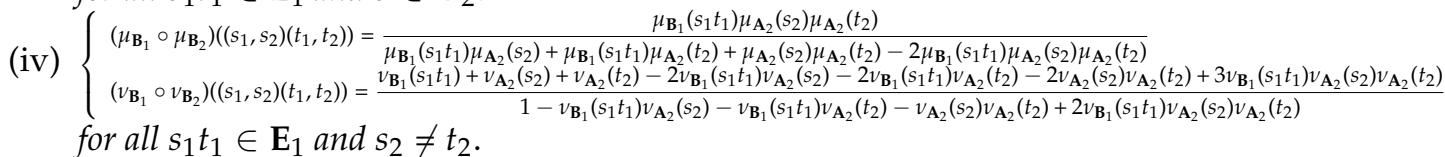

Proposition 10. If $\mathbf{G}_{1}=\left(\mathbf{A}_{1}, \mathbf{B}_{1}\right)$ and $\mathbf{G}_{2}=\left(\mathbf{A}_{2}, \mathbf{B}_{2}\right)$ are PDFEGs of underlying crisp graphs $\mathbf{G}_{1}^{\prime}=\left(\mathbf{V}_{1}, \mathbf{E}_{1}\right)$ and $\mathbf{G}_{2}^{\prime}=\left(\mathbf{V}_{2}, \mathbf{E}_{2}\right)$, respectively, then the composition $\mathbf{G}_{1} \circ \mathbf{G}_{2}$ of $\mathbf{G}_{1}$ and $\mathbf{G}_{2}$ is the PDFEG of $\mathbf{G}_{1}^{\prime} \circ \mathbf{G}_{2}^{\prime}$.

Proof. From Proposition 4 , if $s_{1}=t_{1}=s$ and $s_{2} t_{2} \in \mathbf{E}_{2}$, then we have:

$$
\left(\mu_{\mathbf{B}_{1}} \circ \mu_{\mathbf{B}_{2}}\right)\left(\left(s, s_{2}\right)\left(s, t_{2}\right)\right) \leq \frac{\left(\mu_{\mathbf{A}_{1}} \circ \mu_{\mathbf{A}_{2}}\right)\left(s, s_{2}\right)\left(\mu_{\mathbf{A}_{1}} \circ \mu_{\mathbf{A}_{2}}\right)\left(s, t_{2}\right)}{\left(\mu_{\mathbf{A}_{1}} \circ \mu_{\mathbf{A}_{2}}\right)\left(s, s_{2}\right)+\left(\mu_{\mathbf{A}_{1}} \circ \mu_{\mathbf{A}_{2}}\right)\left(s, t_{2}\right)-\left(\mu_{\mathbf{A}_{1}} \circ \mu_{\mathbf{A}_{2}}\right)\left(s, s_{2}\right)\left(\mu_{\mathbf{A}_{1}} \circ \mu_{\mathbf{A}_{2}}\right)\left(s, t_{2}\right)},
$$




$$
\left(v_{\mathbf{B}_{1}} \circ v_{\mathbf{B}_{2}}\right)\left(\left(s, s_{2}\right)\left(s, t_{2}\right)\right) \leq \frac{\left(v_{\mathbf{A}_{1}} \circ v_{\mathbf{A}_{2}}\right)\left(s, s_{2}\right)+\left(v_{\mathbf{A}_{1}} \circ v_{\mathbf{A}_{2}}\right)\left(s, t_{2}\right)-2\left(v_{\mathbf{A}_{1}} \circ v_{\mathbf{A}_{2}}\right)\left(s, s_{2}\right)\left(v_{\mathbf{A}_{1}} \circ v_{\mathbf{A}_{2}}\right)\left(s, t_{2}\right)}{1-\left(v_{\mathbf{A}_{1}} \circ v_{\mathbf{A}_{2}}\right)\left(s, s_{2}\right)\left(v_{\mathbf{A}_{1}} \circ v_{\mathbf{A}_{2}}\right)\left(s, t_{2}\right)} .
$$

If $s_{1} t_{1} \in \mathbf{E}_{1}$ and $s_{2}=t_{2}=s$, then we have:

$$
\begin{aligned}
& \left(\mu_{\mathbf{B}_{1}} \circ \mu_{\mathbf{B}_{2}}\right)\left(\left(s_{1}, s\right)\left(t_{1}, s\right)\right) \leq \frac{\left(\mu_{\mathbf{A}_{1}} \circ \mu_{\mathbf{A}_{2}}\right)\left(s_{1}, s\right)\left(\mu_{\mathbf{A}_{1}} \circ \mu_{\mathbf{A}_{2}}\right)\left(t_{1}, s\right)}{\left(\mu_{\mathbf{A}_{1}} \circ \mu_{\mathbf{A}_{2}}\right)\left(s_{1}, s\right)+\left(\mu_{\mathbf{A}_{1}} \circ \mu_{\mathbf{A}_{2}}\right)\left(t_{1}, s\right)-\left(\mu_{\mathbf{A}_{1}} \circ \mu_{\mathbf{A}_{2}}\right)\left(s_{1}, s\right)\left(\mu_{\mathbf{A}_{1}} \circ \mu_{\mathbf{A}_{2}}\right)\left(t_{1}, s\right)}, \\
& \left(v_{\mathbf{B}_{1}} \circ v_{\mathbf{B}_{2}}\right)\left(\left(s_{1}, s\right)\left(t_{1}, s\right)\right) \leq \frac{\left(v_{\mathbf{A}_{1}} \circ v_{\mathbf{A}_{2}}\right)\left(s_{1}, s\right)+\left(v_{\mathbf{A}_{1}} \circ v_{\mathbf{A}_{2}}\right)\left(t_{1}, s\right)-2\left(v_{\mathbf{A}_{1}} \circ v_{\mathbf{A}_{2}}\right)\left(s_{1}, s\right)\left(v_{\mathbf{A}_{1}} \circ v_{\mathbf{A}_{2}}\right)\left(t_{1}, s\right)}{1-\left(v_{\mathbf{A}_{1}} \circ v_{\mathbf{A}_{2}}\right)\left(s_{1}, s\right)\left(v_{\mathbf{A}_{1}} \circ v_{\mathbf{A}_{2}}\right)\left(t_{1}, s\right)} .
\end{aligned}
$$

In similar manner, if $s_{1} t_{1} \in \mathbf{E}_{1}$ and $s_{2} \neq t_{2}$, then we have:

$$
\begin{aligned}
\left(\mu_{\mathbf{B}_{1}} \circ \mu_{\mathbf{B}_{2}}\right)\left(\left(s_{1}, s_{2}\right)\left(t_{1}, t_{2}\right)\right)= & \mathcal{T}\left(\mathcal{T}\left(\mu_{\mathbf{A}_{2}}\left(s_{2}\right), \mu_{\mathbf{A}_{2}}\left(t_{2}\right)\right), \mu_{\mathbf{B}_{1}}\left(s_{1} t_{1}\right)\right)=\mathcal{T}\left(\mathcal{T}(1,1), \mu_{\mathbf{B}_{1}}\left(s_{1} t_{1}\right)\right) \\
= & \mathcal{T}\left(1, \mu_{\mathbf{B}_{1}}\left(s_{1} t_{1}\right)\right)=\mu_{\mathbf{B}_{1}}\left(s_{1} t_{1}\right) \leq \frac{\mu_{\mathbf{A}_{1}}\left(s_{1}\right) \mu_{\mathbf{A}_{1}}\left(t_{1}\right)}{\mu_{\mathbf{A}_{1}}\left(s_{1}\right)+\mu_{\mathbf{A}_{1}}\left(t_{1}\right)-\mu_{\mathbf{A}_{1}}\left(s_{1}\right) \mu_{\mathbf{A}_{1}}\left(t_{1}\right)} \\
= & \frac{\left(\mu_{\mathbf{A}_{1}} \circ \mu_{\mathbf{A}_{2}}\right)\left(s_{1}, s_{2}\right)\left(\mu_{\mathbf{A}_{1}} \circ \mu_{\mathbf{A}_{2}}\right)\left(t_{1}, t_{2}\right)}{\left(\mu_{\mathbf{A}_{1}} \circ \mu_{\mathbf{A}_{2}}\right)\left(s_{1}, s_{2}\right)+\left(\mu_{\mathbf{A}_{1}} \circ \mu_{\mathbf{A}_{2}}\right)\left(t_{1}, t_{2}\right)}, \\
& -\left(\mu_{\mathbf{A}_{1}} \circ \mu_{\mathbf{A}_{2}}\right)\left(s_{1}, s_{2}\right)\left(\mu_{\mathbf{A}_{1}} \circ \mu_{\mathbf{A}_{2}}\right)\left(t_{1}, t_{2}\right) \\
\left(v_{\mathbf{B}_{1}} \circ v_{\mathbf{B}_{2}}\right)\left(\left(s_{1}, s_{2}\right)\left(t_{1}, t_{2}\right)\right)= & \mathcal{S}\left(\mathcal{S}\left(v_{\mathbf{A}_{2}}\left(s_{2}\right), v_{\mathbf{A}_{2}}\left(t_{2}\right)\right) v_{\mathbf{B}_{1}}\left(s_{1} t_{1}\right)\right)=\mathcal{S}\left(\mathcal{S}(0,0), v_{\mathbf{B}_{1}}\left(s_{1} t_{1}\right)\right) \\
= & \mathcal{S}\left(0, v_{\mathbf{B}_{1}}\left(s_{1} t_{1}\right)\right)=v_{\mathbf{B}_{1}}\left(s_{1} t_{1}\right) \leq \frac{v_{\mathbf{A}_{1}}\left(s_{1}\right)+v_{\mathbf{A}_{1}}\left(t_{1}\right)-2 v_{\mathbf{A}_{1}}\left(s_{1}\right) v_{\mathbf{A}_{1}}\left(t_{1}\right)}{1-v_{\mathbf{A}_{1}}\left(s_{1}\right) v_{\mathbf{A}_{1}}\left(t_{1}\right)} \\
& \left(v_{\mathbf{A}_{1}} \circ v_{\mathbf{A}_{2}}\right)\left(s_{1}, s_{2}\right)+\left(v_{\mathbf{A}_{1}} \circ v_{\mathbf{A}_{2}}\right)\left(t_{1}, t_{2}\right) \\
= & \frac{-2\left(v_{\mathbf{A}_{1}} \circ v_{\mathbf{A}_{2}}\right)\left(s_{1}, s_{2}\right)\left(v_{\mathbf{A}_{1}} \circ v_{\mathbf{A}_{2}}\right)\left(t_{1}, t_{2}\right)}{1-\left(v_{\mathbf{A}_{1}} \circ v_{\mathbf{A}_{2}}\right)\left(s_{1}, s_{2}\right)\left(v_{\mathbf{A}_{1}} \circ v_{\mathbf{A}_{2}}\right)\left(t_{1}, t_{2}\right)} .
\end{aligned}
$$

Hence, it is concluded that $\mathbf{G}_{1} \circ \mathbf{G}_{2}$ is a PDFEG of $\mathbf{G}_{1}^{\prime} \circ \mathbf{G}_{2}^{\prime}$.

Example 9. Consider PDFEGs $\mathbf{G}_{1}$ and $\mathbf{G}_{2}$ as in Example 3. Then, $\mathbf{G}_{1} \circ \mathbf{G}_{2}$ is displayed in Figure 11.

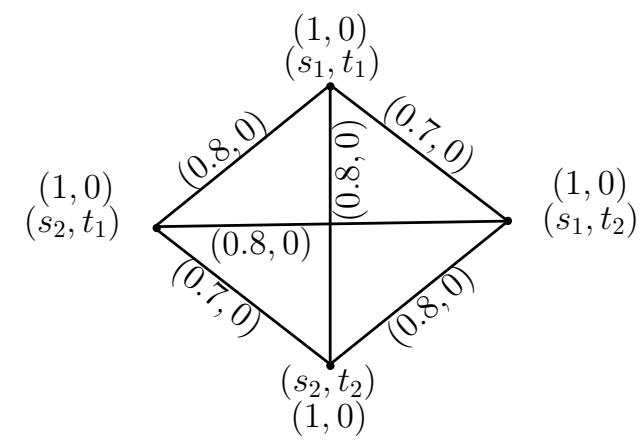

Figure 11. Composition of two PDFEGs.

By routine computations, one can view from Figure 11 that $\mathbf{G}_{1} \circ \mathbf{G}_{2}=\left(\mathbf{A}_{1} \circ \mathbf{A}_{2}, \mathbf{B}_{1} \circ \mathbf{B}_{2}\right)$ is a PDFEG of underlying crisp graph $\mathbf{G}_{1}^{\prime} \circ \mathbf{G}_{2}^{\prime}=\left(\mathbf{V}_{1} \circ \mathbf{V}_{2}, \mathbf{E}_{1} \circ \mathbf{E}_{2}\right)$. 
Definition 15. Consider $\mathbf{G}_{1}=\left(\mathbf{A}_{1}, \mathbf{B}_{1}\right)$ and $\mathbf{G}_{2}=\left(\mathbf{A}_{2}, \mathbf{B}_{2}\right)$ to be two PDFEGs. Then, for any vertex $\left(s_{1}, s_{2}\right) \in \mathbf{V}_{1} \circ \mathbf{V}_{2}$,

$$
\begin{aligned}
& \left(\mathbf{D}_{\mu}\right)_{\mathbf{G}_{1} \circ \mathbf{G}_{2}}\left(s_{1}, s_{2}\right)=\sum_{\left(s_{1}, s_{2}\right)\left(t_{1}, t_{2}\right) \in \mathbf{E}_{1} \circ \mathbf{E}_{2}}\left(\mu_{\mathbf{B}_{1}} \circ \mu_{\mathbf{B}_{2}}\right)\left(\left(s_{1}, s_{2}\right)\left(t_{1}, t_{2}\right)\right) \\
& =\sum_{s_{1}=t_{1}, s_{2} t_{2} \in \mathbf{E}_{2}} \frac{\mu_{\mathbf{A}_{1}}\left(s_{1}\right) \mu_{\mathbf{B}_{2}}\left(s_{2} t_{2}\right)}{\mu_{\mathbf{A}_{1}}\left(s_{1}\right)+\mu_{\mathbf{B}_{2}}\left(s_{2} t_{2}\right)-\mu_{\mathbf{A}_{1}}\left(s_{1}\right) \mu_{\mathbf{B}_{2}}\left(s_{2} t_{2}\right)} \\
& +\sum_{s_{1} t_{1} \in \mathbf{E}_{1}, s_{2}=t_{2}} \frac{\mu_{\mathbf{B}_{1}}\left(s_{1} t_{1}\right) \mu_{\mathbf{A}_{2}}\left(s_{2}\right)}{\mu_{\mathbf{B}_{1}}\left(s_{1} t_{1}\right)+\mu_{\mathbf{A}_{2}}\left(s_{2}\right)-\mu_{\mathbf{B}_{1}}\left(s_{1} t_{1}\right) \mu_{\mathbf{A}_{2}}\left(s_{2}\right)} \\
& +\sum_{s_{1} t_{1} \in \mathbf{E}_{1}, s_{2} \neq t_{2}} \frac{\mu_{\mathbf{B}_{1}}\left(s_{1} t_{1}\right) \mu_{\mathbf{A}_{2}}\left(s_{2}\right) \mu_{\mathbf{A}_{2}}\left(t_{2}\right)}{\mu_{\mathbf{B}_{1}}\left(s_{1} t_{1}\right) \mu_{\mathbf{A}_{2}}\left(s_{2}\right)+\mu_{\mathbf{B}_{1}}\left(s_{1} t_{1}\right) \mu_{\mathbf{A}_{2}}\left(t_{2}\right)+\mu_{\mathbf{A}_{2}}\left(s_{2}\right) \mu_{\mathbf{A}_{2}}\left(t_{2}\right)}, \\
& -2 \mu_{\mathbf{B}_{1}}\left(s_{1} t_{1}\right) \mu_{\mathbf{A}_{2}}\left(s_{2}\right) \mu_{\mathbf{A}_{2}}\left(t_{2}\right) \\
& \left(\mathbf{D}_{v}\right)_{\mathbf{G}_{1} \circ \mathbf{G}_{2}}\left(s_{1}, s_{2}\right)=\sum_{\left(s_{1}, s_{2}\right)\left(t_{1}, t_{2}\right) \in \mathbf{E}_{1} \circ \mathbf{E}_{2}}\left(v_{\mathbf{B}_{1}} \circ v_{\mathbf{B}_{2}}\right)\left(\left(s_{1}, s_{2}\right)\left(t_{1}, t_{2}\right)\right) \\
& =\sum_{s_{1}=t_{1}, s_{2} t_{2} \in \mathbf{E}_{2}} \frac{v_{\mathbf{A}_{1}}\left(s_{1}\right)+v_{\mathbf{B}_{2}}\left(s_{2} t_{2}\right)-2 v_{\mathbf{A}_{1}}\left(s_{1}\right) v_{\mathbf{B}_{2}}\left(s_{2} t_{2}\right)}{1-v_{\mathbf{A}_{1}}\left(s_{1}\right) v_{\mathbf{B}_{2}}\left(s_{2} t_{2}\right)} \\
& +\sum_{s_{1} t_{1} \in \mathbf{E}_{1}, s_{2}=t_{2}} \frac{v_{\mathbf{B}_{1}}\left(s_{1} t_{1}\right)+v_{\mathbf{A}_{2}}\left(s_{2}\right)-2 v_{\mathbf{B}_{1}}\left(s_{1} t_{1}\right) v_{\mathbf{A}_{2}}\left(s_{2}\right)}{1-v_{\mathbf{B}_{1}}\left(s_{1} t_{1}\right) v_{\mathbf{A}_{2}}\left(s_{2}\right)} \\
& v_{\mathbf{B}_{1}}\left(s_{1} t_{1}\right)+v_{\mathbf{A}_{2}}\left(s_{2}\right)+v_{\mathbf{A}_{2}}\left(t_{2}\right)-2 v_{\mathbf{B}_{1}}\left(s_{1} t_{1}\right) v_{\mathbf{A}_{2}}\left(s_{2}\right)-2 v_{\mathbf{B}_{1}}\left(s_{1} t_{1}\right) \\
& +\sum_{s_{1} t_{1} \in \mathbf{E}_{1}, s_{2} \neq t_{2}} \frac{v_{\mathbf{A}_{2}}\left(t_{2}\right)-2 v_{\mathbf{A}_{2}}\left(s_{2}\right) v_{\mathbf{A}_{2}}\left(t_{2}\right)+3 v_{\mathbf{B}_{1}}\left(s_{1} t_{1}\right) v_{\mathbf{A}_{2}}\left(s_{2}\right) v_{\mathbf{A}_{2}}\left(t_{2}\right)}{1-v_{\mathbf{B}_{1}}\left(s_{1} t_{1}\right) v_{\mathbf{A}_{2}}\left(s_{2}\right)-v_{\mathbf{B}_{1}}\left(s_{1} t_{1}\right) v_{\mathbf{A}_{2}}\left(t_{2}\right)-v_{\mathbf{A}_{2}}\left(s_{2}\right) v_{\mathbf{A}_{2}}\left(t_{2}\right)} . \\
& +2 v_{\mathbf{B}_{1}}\left(s_{1} t_{1}\right) v_{\mathbf{A}_{2}}\left(s_{2}\right) v_{\mathbf{A}_{2}}\left(t_{2}\right)
\end{aligned}
$$

Definition 16. Consider $\mathbf{G}_{1}=\left(\mathbf{A}_{1}, \mathbf{B}_{1}\right)$ and $\mathbf{G}_{2}=\left(\mathbf{A}_{2}, \mathbf{B}_{2}\right)$ to be two PDFEGs. Then, for any vertex $\left(s_{1}, s_{2}\right) \in \mathbf{V}_{1} \circ \mathbf{V}_{2}$,

$$
\begin{aligned}
\left(\mathbf{T D}_{\mu}\right)_{\mathbf{G}_{1} \circ \mathbf{G}_{2}}\left(s_{1}, s_{2}\right)= & \sum_{\left(s_{1}, s_{2}\right)\left(t_{1}, t_{2}\right) \in \mathbf{E}_{1} \circ \mathbf{E}_{2}}\left(\mu_{\mathbf{B}_{1}} \circ \mu_{\mathbf{B}_{2}}\right)\left(\left(s_{1}, s_{2}\right)\left(t_{1}, t_{2}\right)\right)+\left(\mu_{\mathbf{A}_{1}} \circ \mu_{\mathbf{A}_{2}}\right)\left(s_{1}, s_{2}\right) \\
= & \sum_{s_{1}=t_{1}, s_{2} t_{2} \in \mathbf{E}_{2}} \frac{\mu_{\mathbf{A}_{1}}\left(s_{1}\right) \mu_{\mathbf{B}_{2}}\left(s_{2} t_{2}\right)}{\mu_{\mathbf{A}_{1}}\left(s_{1}\right)+\mu_{\mathbf{B}_{2}}\left(s_{2} t_{2}\right)-\mu_{\mathbf{A}_{1}}\left(s_{1}\right) \mu_{\mathbf{B}_{2}}\left(s_{2} t_{2}\right)} \\
& +\sum_{s_{1} t_{1} \in \mathbf{E}_{1}, s_{2}=t_{2}} \frac{\mu_{\mathbf{B}_{1}}\left(s_{1} t_{1}\right) \mu_{\mathbf{A}_{2}}\left(s_{2}\right)}{\mu_{\mathbf{B}_{1}}\left(s_{1} t_{1}\right)+\mu_{\mathbf{A}_{2}}\left(s_{2}\right)-\mu_{\mathbf{B}_{1}}\left(s_{1} t_{1}\right) \mu_{\mathbf{A}_{2}}\left(s_{2}\right)} \\
& +\sum_{s_{1} t_{1} \in \mathbf{E}_{1}, s_{2} \neq t_{2}} \frac{\mu_{\mathbf{B}_{1}}\left(s_{1} t_{1}\right) \mu_{\mathbf{A}_{2}}\left(s_{2}\right) \mu_{\mathbf{A}_{2}}\left(t_{2}\right)}{\mu_{\mathbf{B}_{1}}\left(s_{1} t_{1}\right) \mu_{\mathbf{A}_{2}}\left(s_{2}\right)+\mu_{\mathbf{B}_{1}}\left(s_{1} t_{1}\right) \mu_{\mathbf{A}_{2}}\left(t_{2}\right)+\mu_{\mathbf{A}_{2}}\left(s_{2}\right) \mu_{\mathbf{A}_{2}}\left(t_{2}\right)} \\
& +\frac{-2 \mu_{\mathbf{B}_{1}}\left(s_{1} t_{1}\right) \mu_{\mathbf{A}_{2}}\left(s_{2}\right) \mu_{\mathbf{A}_{2}}\left(t_{2}\right)}{\mu_{\mathbf{A}_{1}}\left(s_{1}\right)+\mu_{\mathbf{A}_{2}}\left(s_{2}\right)-\mu_{\mathbf{A}_{1}}\left(s_{1}\right) \mu_{\mathbf{A}_{2}}\left(s_{2}\right)}
\end{aligned}
$$




$$
\begin{aligned}
\left(\mathbf{T D}_{v}\right)_{\mathbf{G}_{1} \circ \mathbf{G}_{2}}\left(s_{1}, s_{2}\right)= & \sum_{\left(s_{1}, s_{2}\right)\left(t_{1}, t_{2}\right) \in \mathbf{E}_{1} \circ \mathbf{E}_{2}}\left(v_{\mathbf{B}_{1}} \circ v_{\mathbf{B}_{2}}\right)\left(\left(s_{1}, s_{2}\right)\left(t_{1}, t_{2}\right)\right)+\left(v_{\mathbf{A}_{1}} \circ v_{\mathbf{A}_{2}}\right)\left(s_{1}, s_{2}\right) \\
= & \sum_{s_{1}=t_{1}, s_{2} t_{2} \in \mathbf{E}_{2}} \frac{v_{\mathbf{A}_{1}}\left(s_{1}\right)+v_{\mathbf{B}_{2}}\left(s_{2} t_{2}\right)-2 v_{\mathbf{A}_{1}}\left(s_{1}\right) v_{\mathbf{B}_{2}}\left(s_{2} t_{2}\right)}{1-v_{\mathbf{A}_{1}}\left(s_{1}\right) v_{\mathbf{B}_{2}}\left(s_{2} t_{2}\right)} \\
& +\sum_{s_{1} t_{1} \in \mathbf{E}_{1}, s_{2}=t_{2}} \frac{v_{\mathbf{B}_{1}}\left(s_{1} t_{1}\right)+v_{\mathbf{A}_{2}}\left(s_{2}\right)-2 v_{\mathbf{B}_{1}}\left(s_{1} t_{1}\right) v_{\mathbf{A}_{2}}\left(s_{2}\right)}{1-v_{\mathbf{B}_{1}}\left(s_{1} t_{1}\right) v_{\mathbf{A}_{2}}\left(s_{2}\right)} \\
& +\sum_{s_{1} t_{1} \in \mathbf{E}_{1}, s_{2} \neq t_{2}} \frac{v_{\mathbf{B}_{1}}\left(s_{1} t_{1}\right)+v_{\mathbf{A}_{2}}\left(s_{2}\right)+v_{\mathbf{A}_{2}}\left(t_{2}\right)-2 v_{\mathbf{B}_{1}}\left(s_{1} t_{1}\right) v_{\mathbf{A}_{2}}\left(s_{2}\right)-2 v_{\mathbf{B}_{1}}\left(s_{1} t_{1}\right)}{1-v_{\mathbf{B}_{1}}\left(s_{1} t_{1}\right) v_{\mathbf{A}_{2}}\left(s_{2}\right)-v_{\mathbf{B}_{1}}\left(s_{1} t_{1}\right) v_{\mathbf{A}_{2}}\left(t_{2}\right)-v_{\mathbf{A}_{2}}\left(s_{2}\right) v_{\mathbf{A}_{2}}\left(t_{2}\right)} \\
& +\frac{v_{\mathbf{A}_{1}}\left(s_{1}\right)+v_{\mathbf{A}_{2}}\left(s_{2}\right)-2 v_{\mathbf{A}_{1}}\left(s_{1}\right) v_{\mathbf{A}_{2}}\left(s_{2}\right)}{1-v_{\mathbf{A}_{1}}\left(s_{1}\right) v_{\mathbf{A}_{2}}\left(s_{2}\right)} .
\end{aligned}
$$

Example 10. Consider PDFEGs $\mathbf{G}_{1}$ and $\mathbf{G}_{2}$ as in Example 3; their composition is given in Figure 11. Then, by Definition 15, we must have:

$$
\begin{aligned}
& \left(\mathbf{D}_{\mu}\right)_{\mathbf{G}_{1} \circ \mathbf{G}_{2}}\left(s_{1}, t_{1}\right)=\begin{array}{l}
\left(\mu_{\mathbf{B}_{1}} \circ \mu_{\mathbf{B}_{2}}\right)\left(\left(s_{1}, t_{1}\right)\left(s_{1}, t_{2}\right)\right)+\left(\mu_{\mathbf{B}_{1}} \circ \mu_{\mathbf{B}_{2}}\right)\left(\left(s_{1}, t_{1}\right)\left(s_{2}, t_{1}\right)\right)+\left(\mu_{\mathbf{B}_{1}} \circ \mu_{\mathbf{B}_{2}}\right)\left(\left(s_{1}, t_{1}\right)\right. \\
\left.\left(s_{2}, t_{2}\right)\right)
\end{array} \\
& =\frac{\mu_{\mathbf{A}_{1}}\left(s_{1}\right) \mu_{\mathbf{B}_{2}}\left(t_{1} t_{2}\right)}{\mu_{\mathbf{A}_{1}}\left(s_{1}\right)+\mu_{\mathbf{B}_{2}}\left(t_{1} t_{2}\right)-\mu_{\mathbf{A}_{1}}\left(s_{1}\right) \mu_{\mathbf{B}_{2}}\left(t_{1} t_{2}\right)}+\frac{\mu_{\mathbf{B}_{1}}\left(s_{1} s_{2}\right) \mu_{\mathbf{A}_{2}}\left(t_{1}\right)}{\mu_{\mathbf{B}_{1}}\left(s_{1} s_{2}\right)+\mu_{\mathbf{A}_{2}}\left(t_{1}\right)-\mu_{\mathbf{B}_{1}}\left(s_{1} s_{2}\right) \mu_{\mathbf{A}_{2}}\left(t_{1}\right)} \\
& +\frac{\mu_{\mathbf{B}_{1}}\left(s_{1} t_{1}\right) \mu_{\mathbf{A}_{2}}\left(s_{2}\right) \mu_{\mathbf{A}_{2}}\left(t_{2}\right)}{\mu_{\mathbf{B}_{1}}\left(s_{1} t_{1}\right) \mu_{\mathbf{A}_{2}}\left(s_{2}\right)+\mu_{\mathbf{B}_{1}}\left(s_{1} t_{1}\right) \mu_{\mathbf{A}_{2}}\left(t_{2}\right)+\mu_{\mathbf{A}_{2}}\left(s_{2}\right) \mu_{\mathbf{A}_{2}}\left(t_{2}\right)} \\
& -2 \mu_{\mathbf{B}_{1}}\left(s_{1} t_{1}\right) \mu_{\mathbf{A}_{2}}\left(s_{2}\right) \mu_{\mathbf{A}_{2}}\left(t_{2}\right) \\
& =0.7+0.8+0.8=2.3 \text {, } \\
& \left(\mathbf{D}_{v}\right)_{\mathbf{G}_{1} \circ \mathbf{G}_{2}}\left(s_{1}, t_{1}\right)=\begin{array}{l}
\left(v_{\mathbf{B}_{1}} \circ v_{\mathbf{B}_{2}}\right)\left(\left(s_{1}, t_{1}\right)\left(s_{1}, t_{2}\right)\right)+\left(v_{\mathbf{B}_{1}} \circ v_{\mathbf{B}_{2}}\right)\left(\left(s_{1}, t_{1}\right)\left(s_{2}, t_{1}\right)\right)+\left(v_{\mathbf{B}_{1}} \circ v_{\mathbf{B}_{2}}\right)\left(\left(s_{1}, t_{1}\right)\right. \\
\left.\left(s_{2}, t_{2}\right)\right)
\end{array} \\
& =\frac{v_{\mathbf{A}_{1}}\left(s_{1}\right)+v_{\mathbf{B}_{2}}\left(t_{1} t_{2}\right)-2 v_{\mathbf{A}_{1}}\left(s_{1}\right) v_{\mathbf{B}_{2}}\left(t_{1} t_{2}\right)}{1-v_{\mathbf{A}_{1}}\left(s_{1}\right) v_{\mathbf{B}_{2}}\left(t_{1} t_{2}\right)}+\frac{v_{\mathbf{B}_{1}}\left(s_{1} s_{2}\right)+v_{\mathbf{A}_{2}}\left(t_{1}\right)-2 v_{\mathbf{B}_{1}}\left(s_{1} s_{2}\right) v_{\mathbf{A}_{2}}\left(t_{1}\right)}{1-v_{\mathbf{B}_{1}}\left(s_{1} s_{2}\right) v_{\mathbf{A}_{2}}\left(t_{1}\right)} \\
& v_{\mathbf{B}_{1}}\left(s_{1} t_{1}\right)+v_{\mathbf{A}_{2}}\left(s_{2}\right)+v_{\mathbf{A}_{2}}\left(t_{2}\right)-2 v_{\mathbf{B}_{1}}\left(s_{1} t_{1}\right) v_{\mathbf{A}_{2}}\left(s_{2}\right)-2 v_{\mathbf{B}_{1}}\left(s_{1} t_{1}\right) v_{\mathbf{A}_{2}}\left(t_{2}\right) \\
& +\frac{-2 v_{\mathbf{A}_{2}}\left(s_{2}\right) v_{\mathbf{A}_{2}}\left(t_{2}\right)+3 v_{\mathbf{B}_{1}}\left(s_{1} t_{1}\right) v_{\mathbf{A}_{2}}\left(s_{2}\right) v_{\mathbf{A}_{2}}\left(t_{2}\right)}{1-v_{\mathbf{B}_{1}}\left(s_{1} t_{1}\right) v_{\mathbf{A}_{2}}\left(s_{2}\right)-v_{\mathbf{B}_{1}}\left(s_{1} t_{1}\right) v_{\mathbf{A}_{2}}\left(t_{2}\right)-v_{\mathbf{A}_{2}}\left(s_{2}\right) v_{\mathbf{A}_{2}}\left(t_{2}\right)} \\
& +2 v_{\mathbf{B}_{1}}\left(s_{1} t_{1}\right) v_{\mathbf{A}_{2}}\left(s_{2}\right) v_{\mathbf{A}_{2}}\left(t_{2}\right) \\
& =0+0+0=0 .
\end{aligned}
$$

Thus, $(\mathbf{D})_{\mathbf{G}_{1} \circ \mathbf{G}_{2}}\left(s_{1}, t_{1}\right)=(2 \cdot 3,0)$.

Further, by using Definition 16, we have:

$$
\begin{aligned}
\left(\mathbf{T D}_{\mu}\right)_{\mathbf{G}_{1} \circ \mathbf{G}_{2}}\left(s_{1}, t_{1}\right) & =\left(\mathbf{D}_{\mu}\right)_{\mathbf{G}_{1} \circ \mathbf{G}_{2}}\left(s_{1}, t_{1}\right)+\left(\mu_{\mathbf{A}_{1}} \circ \mu_{\mathbf{A}_{2}}\right)\left(s_{1}, t_{1}\right) \\
& =2.3+\frac{\mu_{\mathbf{A}_{1}}\left(s_{1}\right) \mu_{\mathbf{A}_{2}}\left(t_{1}\right)}{\mu_{\mathbf{A}_{1}}\left(s_{1}\right)+\mu_{\mathbf{A}_{2}}\left(t_{1}\right)-\mu_{\mathbf{A}_{1}}\left(s_{1}\right) \mu_{\mathbf{A}_{2}}\left(t_{1}\right)}=3.3, \\
\left(\mathbf{T D}_{v}\right)_{\mathbf{G}_{1} \circ \mathbf{G}_{2}}\left(s_{1}, t_{1}\right) & =\left(\mathbf{D}_{v}\right)_{\mathbf{G}_{1} \circ \mathbf{G}_{2}}\left(s_{1}, t_{1}\right)+\left(v_{\mathbf{A}_{1}} \circ v_{\mathbf{A}_{2}}\right)\left(s_{1}, t_{1}\right) \\
& =0+\frac{v_{\mathbf{A}_{1}}\left(s_{1}\right)+v_{\mathbf{A}_{2}}\left(t_{1}\right)-2 v_{\mathbf{A}_{1}}\left(s_{1}\right) v_{\mathbf{A}_{2}}\left(t_{1}\right)}{1-v_{\mathbf{A}_{1}}\left(s_{1}\right) v_{\mathbf{A}_{2}}\left(t_{1}\right)}=0 .
\end{aligned}
$$


Thus, $(\mathbf{T D})_{\mathbf{G}_{1} \circ \mathbf{G}_{2}}\left(s_{1}, t_{1}\right)=(3.3,0)$.

Remark 5. The composition $\mathbf{G}_{1} \circ \mathbf{G}_{2}$ of strong PDFGs $\mathbf{G}_{1}$ and $\mathbf{G}_{2}$ is not a PDFG. It is justified in the following example.

Consider strong PDFGs $\mathbf{G}_{1}$ and $\mathbf{G}_{2}$. Their $\mathbf{G}_{1} \circ \mathbf{G}_{2}$ is displayed in Figure 12.

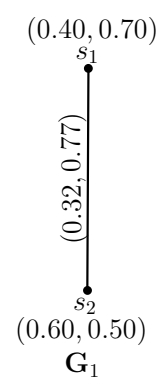

$\mathbf{G}_{1}$

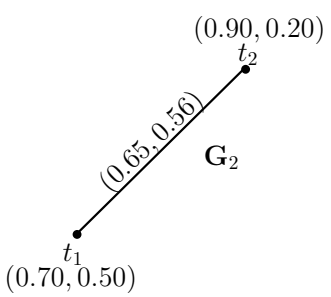

$(0.70,0.50)$

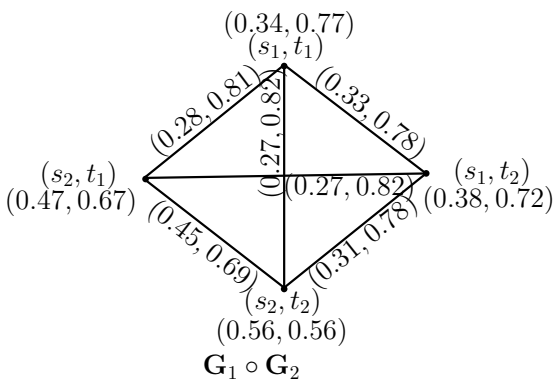

$\mathbf{G}_{1} \circ \mathbf{G}_{2}$

Figure 12. $\mathrm{G}_{1} \circ \mathrm{G}_{2}$ is not a PDFG.

Since for the membership and non-membership grade of $\left(s_{1}, t_{1}\right)\left(s_{1}, t_{2}\right)$ :

$$
\begin{aligned}
& \left(\mu_{\mathbf{B}_{1}} \circ \mu_{\mathbf{B}_{2}}\right)\left(\left(s_{1}, t_{1}\right)\left(s_{1}, t_{2}\right)\right)=0.33 \not \leq 0.22=\frac{\left(\mu_{\mathbf{A}_{1}} \circ \mu_{\mathbf{A}_{2}}\right)\left(s_{1}, t_{1}\right)\left(\mu_{\mathbf{A}_{1}} \circ \mu_{\mathbf{A}_{2}}\right)\left(s_{1}, t_{2}\right)}{\left(\mu_{\mathbf{A}_{1}} \circ \mu_{\mathbf{A}_{2}}\right)\left(s_{1}, t_{1}\right)+\left(\mu_{\mathbf{A}_{1}} \circ \mu_{\mathbf{A}_{2}}\right)\left(s_{1}, t_{2}\right)}, \\
& -\left(\mu_{\mathbf{A}_{1}} \circ \mu_{\mathbf{A}_{2}}\right)\left(s_{1}, t_{1}\right)\left(\mu_{\mathbf{A}_{1}} \circ \mu_{\mathbf{A}_{2}}\right)\left(s_{1}, t_{2}\right) \\
& \left(v_{\mathbf{A}_{1}} \circ v_{\mathbf{A}_{2}}\right)\left(s_{1}, t_{1}\right)+\left(v_{\mathbf{A}_{1}} \circ v_{\mathbf{A}_{2}}\right)\left(s_{1}, t_{2}\right) \\
& \left(v_{\mathbf{B}_{1}} \circ v_{\mathbf{B}_{2}}\right)\left(\left(s_{1}, t_{1}\right)\left(s_{1}, t_{2}\right)\right)=0.78 \leq 0.86=\frac{-2\left(v_{\mathbf{A}_{1}} \circ v_{\mathbf{A}_{2}}\right)\left(s_{1}, t_{1}\right)\left(v_{\mathbf{A}_{1}} \circ v_{\mathbf{A}_{2}}\right)\left(s_{1}, t_{2}\right)}{1-\left(v_{\mathbf{A}_{1}} \circ v_{\mathbf{A}_{2}}\right)\left(s_{1}, t_{1}\right)\left(v_{\mathbf{A}_{1}} \circ v_{\mathbf{A}_{2}}\right)\left(s_{1}, t_{2}\right)} .
\end{aligned}
$$

Likewise, for the membership and non-membership grade of $\left(s_{1}, t_{1}\right)\left(s_{2}, t_{1}\right)$ :

$$
\begin{aligned}
& \left(\mu_{\mathbf{B}_{1}} \circ \mu_{\mathbf{B}_{2}}\right)\left(\left(s_{1}, t_{1}\right)\left(s_{2}, t_{1}\right)\right)=0.28 \not 0.25=\frac{\left(\mu_{\mathbf{A}_{1}} \circ \mu_{\mathbf{A}_{2}}\right)\left(s_{1}, t_{1}\right)\left(\mu_{\mathbf{A}_{1}} \circ \mu_{\mathbf{A}_{2}}\right)\left(s_{2}, t_{1}\right)}{\left(\mu_{\mathbf{A}_{1}} \circ \mu_{\mathbf{A}_{2}}\right)\left(s_{1}, t_{1}\right)+\left(\mu_{\mathbf{A}_{1}} \circ \mu_{\mathbf{A}_{2}}\right)\left(s_{2}, t_{1}\right)}, \\
& -\left(\mu_{\mathbf{A}_{1}} \circ \mu_{\mathbf{A}_{2}}\right)\left(s_{1}, t_{1}\right)\left(\mu_{\mathbf{A}_{1}} \circ \mu_{\mathbf{A}_{2}}\right)\left(s_{2}, t_{1}\right) \\
& \left(v_{\mathbf{A}_{1}} \circ v_{\mathbf{A}_{2}}\right)\left(s_{1}, t_{1}\right)+\left(v_{\mathbf{A}_{1}} \circ v_{\mathbf{A}_{2}}\right)\left(s_{2}, t_{1}\right) \\
& \left(v_{\mathbf{B}_{1}} \circ v_{\mathbf{B}_{2}}\right)\left(\left(s_{1}, t_{1}\right)\left(s_{2}, t_{1}\right)\right)=0.81 \leq 0.84=\frac{-2\left(v_{\mathbf{A}_{1}} \circ v_{\mathbf{A}_{2}}\right)\left(s_{1}, t_{1}\right)\left(v_{\mathbf{A}_{1}} \circ v_{\mathbf{A}_{2}}\right)\left(s_{2}, t_{1}\right)}{1-\left(v_{\mathbf{A}_{1}} \circ v_{\mathbf{A}_{2}}\right)\left(s_{1}, t_{1}\right)\left(v_{\mathbf{A}_{1}} \circ v_{\mathbf{A}_{2}}\right)\left(s_{2}, t_{1}\right)} .
\end{aligned}
$$

Further, for the membership and non-membership grade of $\left(s_{1}, t_{1}\right)\left(s_{2}, t_{2}\right)$ :

$$
\begin{aligned}
& \left(\mu_{\mathbf{B}_{1}} \circ \mu_{\mathbf{B}_{2}}\right)\left(\left(s_{1}, t_{1}\right)\left(s_{2}, t_{2}\right)\right)=0.27=0.27=\frac{\left(\mu_{\mathbf{A}_{1}} \circ \mu_{\mathbf{A}_{2}}\right)\left(s_{1}, t_{1}\right)\left(\mu_{\mathbf{A}_{1}} \circ \mu_{\mathbf{A}_{2}}\right)\left(s_{2}, t_{2}\right)}{\left(\mu_{\mathbf{A}_{1}} \circ \mu_{\mathbf{A}_{2}}\right)\left(s_{1}, t_{1}\right)+\left(\mu_{\mathbf{A}_{1}} \circ \mu_{\mathbf{A}_{2}}\right)\left(s_{2}, t_{2}\right)}, \\
& -\left(\mu_{\mathbf{A}_{1}} \circ \mu_{\mathbf{A}_{2}}\right)\left(s_{1}, t_{1}\right)\left(\mu_{\mathbf{A}_{1}} \circ \mu_{\mathbf{A}_{2}}\right)\left(s_{2}, t_{2}\right) \\
& \left(v_{\mathbf{A}_{1}} \circ v_{\mathbf{A}_{2}}\right)\left(s_{1}, t_{1}\right)+\left(v_{\mathbf{A}_{1}} \circ v_{\mathbf{A}_{2}}\right)\left(s_{2}, t_{2}\right) \\
& \left(v_{\mathbf{B}_{1}} \circ v_{\mathbf{B}_{2}}\right)\left(\left(s_{1}, t_{1}\right)\left(s_{2}, t_{2}\right)\right)=0.82=0.82=\frac{-2\left(v_{\mathbf{A}_{1}} \circ v_{\mathbf{A}_{2}}\right)\left(s_{1}, t_{1}\right)\left(v_{\mathbf{A}_{1}} \circ v_{\mathbf{A}_{2}}\right)\left(s_{2}, t_{2}\right)}{1-\left(v_{\mathbf{A}_{1}} \circ v_{\mathbf{A}_{2}}\right)\left(s_{1}, t_{1}\right)\left(v_{\mathbf{A}_{1}} \circ v_{\mathbf{A}_{2}}\right)\left(s_{2}, t_{2}\right)} .
\end{aligned}
$$


Hence, it is concluded that $\mathbf{G}_{1} \circ \mathbf{G}_{2}$ is not a PDFG of underlying crisp graph $\mathbf{G}_{1}^{\prime} \circ \mathbf{G}_{2}^{\prime}$.

Proposition 11. If $\mathbf{G}_{1}=\left(\mathbf{A}_{1}, \mathbf{B}_{1}\right)$ and $\mathbf{G}_{2}=\left(\mathbf{A}_{2}, \mathbf{B}_{2}\right)$ are strong PDFEGs of underlying crisp graphs $\mathbf{G}_{1}^{\prime}=$ $\left(\mathbf{V}_{1}, E_{1}\right)$ and $\mathbf{G}_{2}^{\prime}=\left(\mathbf{V}_{2}, E_{2}\right)$, respectively, then the composition $\mathbf{G}_{1} \circ \mathbf{G}_{2}$ of $\mathbf{G}_{1}$ and $\mathbf{G}_{2}$ is also a strong PDFEG of $\mathbf{G}_{1}^{\prime} \circ \mathbf{G}_{2}^{\prime}$.

\section{Numerical Approach}

In this section, we solve a decision-making problem concerning the "selection of a leading textile industry" to clarify the suitability of the proposed concept of PDFGs in a realistic scenario. Within the Pythagorean fuzzy preference relation (PFPR) [29] framework, the algorithm for the selection of a leading textile industry is summarized in Algorithm 1. Further, a comparison with existing MCDM techniques is given in Table 7, which interprets the authenticity of our proposed technique.

\subsection{Selection of a Leading Textile Industry}

The clothing and textile industry is very essential in social and economic terms for the growth and development of various countries. According to existing trends, the ability of planning and designing clothes, footwear, and accessories is a pivotal tool for any leading industry. To contribute to the long term development, the capability of the textile industry depends on the criteria of investors, as well as the quality of their items and products. Different places have their unique trends of fabric, and this varies with the passage of time. A newly graduated designer is planning to start her boutique in a town. As the fabric itself is the most integral part, therefore on account of the fine fabric, she pays attention to four textile industries $\mathbf{F}_{l}(l=1,2,3,4)$ that are doing really well on the market. To select the finest option among all industries with limited effort and time, she discusses this matter with an analytical textile technologist $\mathbf{E}$. The decision-making expert makes a comparison between four industries with respect to four criteria $\mathbf{C}_{g}(g=1,2,3,4)$ which are given as:

$\mathrm{C}_{1}=$ durability of fabric;

$\mathrm{C}_{2}=$ price of fabric;

$\mathrm{C}_{3}=$ moisture absorption and heat conductivity;

$\mathrm{C}_{4}=$ appearance and style of the fabric;

with the respective weight vector $\mathbf{W}=(0.4,0.3,0.2,0.1)^{\mathcal{T}}$ and presents his preferable information (PFPRs [29]) $\mathbf{Q}^{(g)}=\left(q_{l p}^{(g)}\right)_{4 \times 4}(g=1,2,3,4)$, where $q_{l p}^{(g)}=\left(\mu_{l p}^{(g)}, v_{l p}^{(g)}\right)$ is the PFN assigned by the decision-making expert with $\mu_{l p}$ and $v_{l p}$ as the degree to which the textile industry $\mathbf{F}_{l}$ is preferred and not preferred over the textile industry $\mathbf{F}_{p}$ regarding the given criteria, respectively. The PFPRs $\mathbf{Q}^{(g)}=\left(q_{l p}^{(g)}\right)_{4 \times 4}$ are outlined in the following tables (Tables 1-4).

Table 1. Pythagorean fuzzy preference relation (PFPR) regarding the criterion "durability of fabric".

\begin{tabular}{ccccc}
\hline $\mathbf{Q}^{(1)}$ & $\mathbf{F}_{1}$ & $\mathbf{F}_{2}$ & $\mathbf{F}_{3}$ & $\mathbf{F}_{4}$ \\
\hline $\mathbf{F}_{1}$ & $(0.5,0.5)$ & $(0.8,0.2)$ & $(0.9,0.1)$ & $(0.8,0.1)$ \\
$\mathbf{F}_{2}$ & $(0.2,0.8)$ & $(0.5,0.5)$ & $(0.7,0.3)$ & $(0.5,0.7)$ \\
$\mathbf{F}_{3}$ & $(0.1,0.9)$ & $(0.3,0.7)$ & $(0.5,0.5)$ & $(0.3,0.8)$ \\
$\mathbf{F}_{4}$ & $(0.1,0.8)$ & $(0.7,0.5)$ & $(0.8,0.3)$ & $(0.5,0.5)$ \\
\hline
\end{tabular}


Table 2. PFPR regarding the criterion "price of fabric".

\begin{tabular}{ccccc}
\hline $\mathbf{Q}^{(2)}$ & $\mathbf{F}_{1}$ & $\mathbf{F}_{2}$ & $\mathbf{F}_{3}$ & $\mathbf{F}_{4}$ \\
\hline $\mathbf{F}_{1}$ & $(0.5,0.5)$ & $(0.7,0.3)$ & $(0.9,0.2)$ & $(0.8,0.2)$ \\
$\mathbf{F}_{2}$ & $(0.3,0.7)$ & $(0.5,0.5)$ & $(0.8,0.1)$ & $(0.6,0.6)$ \\
$\mathbf{F}_{3}$ & $(0.2,0.9)$ & $(0.1,0.8)$ & $(0.5,0.5)$ & $(0.1,0.9)$ \\
$\mathbf{F}_{4}$ & $(0.2,0.8)$ & $(0.6,0.6)$ & $(0.9,0.1)$ & $(0.5,0.5)$ \\
\hline
\end{tabular}

Table 3. PFPR regarding the criterion "moisture absorption and heat conductivity".

\begin{tabular}{ccccc}
\hline $\mathbf{Q}^{(3)}$ & $\mathbf{F}_{1}$ & $\mathbf{F}_{2}$ & $\mathbf{F}_{3}$ & $\mathbf{F}_{4}$ \\
\hline $\mathbf{F}_{1}$ & $(0.5,0.5)$ & $(0.7,0.2)$ & $(0.8,0.1)$ & $(0.7,0.2)$ \\
$\mathbf{F}_{2}$ & $(0.2,0.7)$ & $(0.5,0.5)$ & $(0.5,0.6)$ & $(0.4,0.7)$ \\
$\mathbf{F}_{3}$ & $(0.1,0.8)$ & $(0.6,0.5)$ & $(0.5,0.5)$ & $(0.1,0.9)$ \\
$\mathbf{F}_{4}$ & $(0.2,0.7)$ & $(0.7,0.4)$ & $(0.9,0.1)$ & $(0.5,0.5)$ \\
\hline
\end{tabular}

Table 4. PFPR regarding the criterion "appearance and style of the fabric".

\begin{tabular}{ccccc}
\hline $\mathbf{Q}^{(4)}$ & $\mathbf{F}_{1}$ & $\mathbf{F}_{2}$ & $\mathbf{F}_{3}$ & $\mathbf{F}_{4}$ \\
\hline $\mathbf{F}_{1}$ & $(0.5,0.5)$ & $(0.8,0.3)$ & $(0.9,0.2)$ & $(0.8,0.2)$ \\
$\mathbf{F}_{2}$ & $(0.3,0.8)$ & $(0.5,0.5)$ & $(0.4,0.7)$ & $(0.3,0.9)$ \\
$\mathbf{F}_{3}$ & $(0.2,0.9)$ & $(0.7,0.4)$ & $(0.5,0.5)$ & $(0.5,0.6)$ \\
$\mathbf{F}_{4}$ & $(0.2,0.8)$ & $(0.9,0.3)$ & $(0.6,0.5)$ & $(0.5,0.5)$ \\
\hline
\end{tabular}

The Pythagorean fuzzy directed network (PFDN) $\mathbf{D}_{g}$ concerning PFPRs $\mathbf{Q}^{(g)}(g=1,2,3,4)$ provided in Tables 1-4 is displayed in Figure 13.

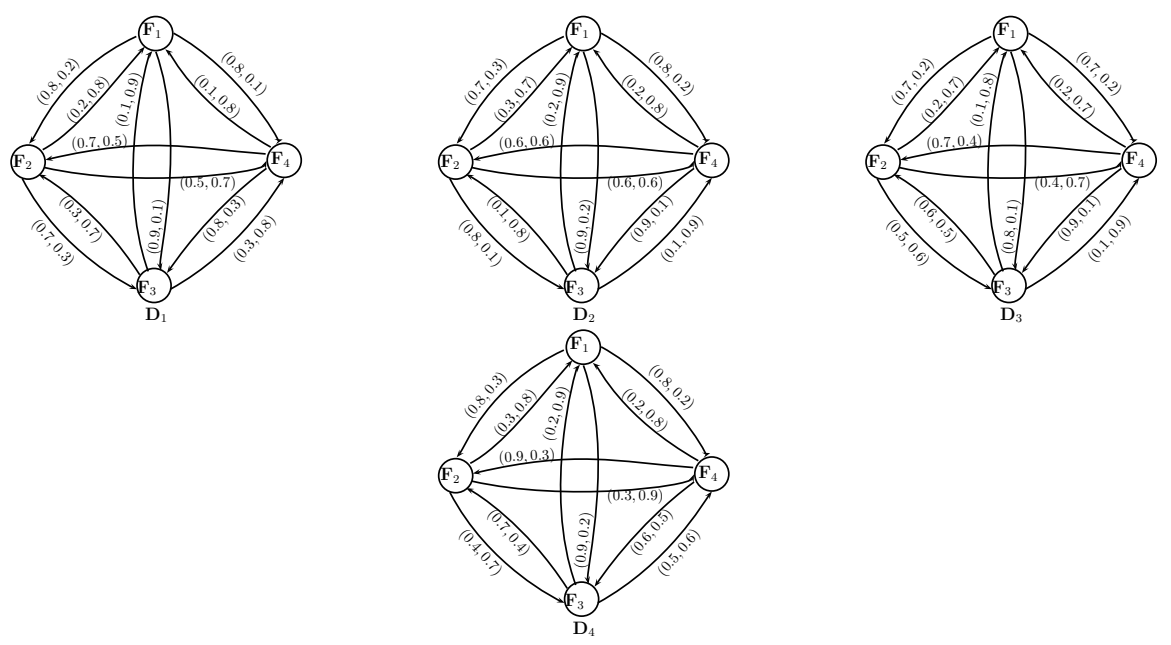

Figure 13. Directed network of PFPRs.

With the purpose to compute the clumped PFN $q_{l p}=\left(\mu_{l p}, v_{l p}\right)(l, p=1,2,3,4)$ of the textile industry $\mathbf{F}_{l}$ over the textile industry $\mathbf{F}_{p}$ regarding all considered criteria $\mathbf{C}^{(g)}(g=1,2,3,4)$, the Pythagorean Dombi fuzzy weighted arithmetic averaging (PDFWAA) operator [47] defined in Equation (1) is utilized.

$$
q_{l p}=\operatorname{PDFWAA}\left(q_{l p}^{(1)}, q_{l p}^{(2)}, \ldots, q_{l p}^{(n)}\right)
$$




$$
=\left(\sqrt{1-\frac{1}{1+\left[\sum_{g=1}^{n} \mathbf{W}_{g}\left(\frac{\left(\mu_{l p}^{(g)}\right)^{2}}{1-\left(\mu_{l p}^{(g)}\right)^{2}}\right)^{\gamma}\right]^{\frac{1}{\gamma}}}}, \frac{1}{1+\left[\sum_{g=1}^{n} \mathbf{W}_{g}\left(\frac{1-v_{l p}^{(g)}}{v_{l p}^{(g)}}\right)^{\gamma}\right]^{\frac{1}{\gamma}}}\right) .
$$

In Equation 1, we considered $\gamma=1$ as in Dombi's t-norm and t-conorm, and we have chosen $\gamma=1$, to obtain the corresponding clumped PFPR $\mathbf{Q}=\left(q_{l p}\right)_{4 \times 4}$, which is shown in Table 5 .

Table 5. Clumped Pythagorean fuzzy preference relation.

\begin{tabular}{ccccc}
\hline $\mathbf{Q}$ & $\mathbf{F}_{1}$ & $\mathbf{F}_{2}$ & $\mathbf{F}_{3}$ & $\mathbf{F}_{4}$ \\
\hline $\mathbf{F}_{1}$ & $(0.5000,0.5000)$ & $(0.7602,0.2308)$ & $(0.8889,0.1250)$ & $(0.7858,0.1429)$ \\
$\mathbf{F}_{2}$ & $(0.2463,0.7467)$ & $(0.5000,0.5000)$ & $(0.7077,0.2079)$ & $(0.5092,0.6811)$ \\
$\mathbf{F}_{3}$ & $(0.1490,0.8781)$ & $(0.4481,0.6264)$ & $(0.5000,0.5000)$ & $(0.2688,0.8182)$ \\
$\mathbf{F}_{4}$ & $(0.1682,0.7778)$ & $(0.7345,0.4687)$ & $(0.8623,0.1531)$ & $(0.5000,0.5000)$ \\
\hline
\end{tabular}

The PFDN D, corresponding to clumped PFPR Q, is drawn in Figure 14.

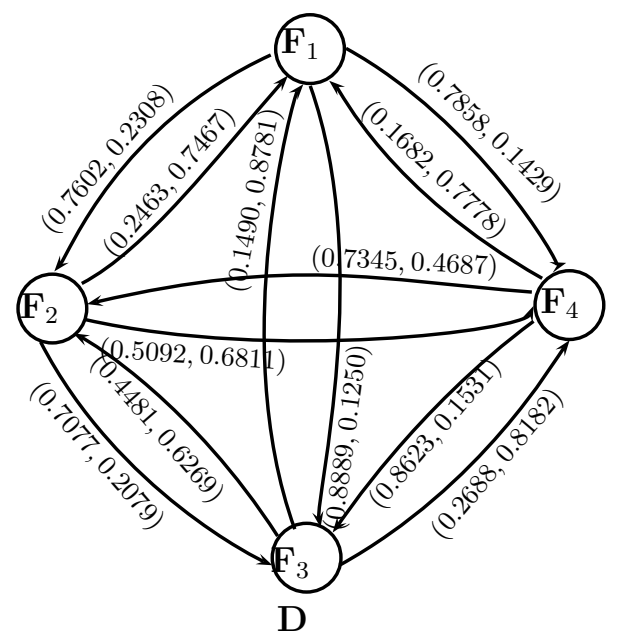

Figure 14. Directed network of clumped PFPR.

Under the condition $\mu_{l p} \geq 0.5(l, p=1,2,3,4)$, a partial directed network $\dot{\mathbf{D}}$ is drawn in Figure 15. 


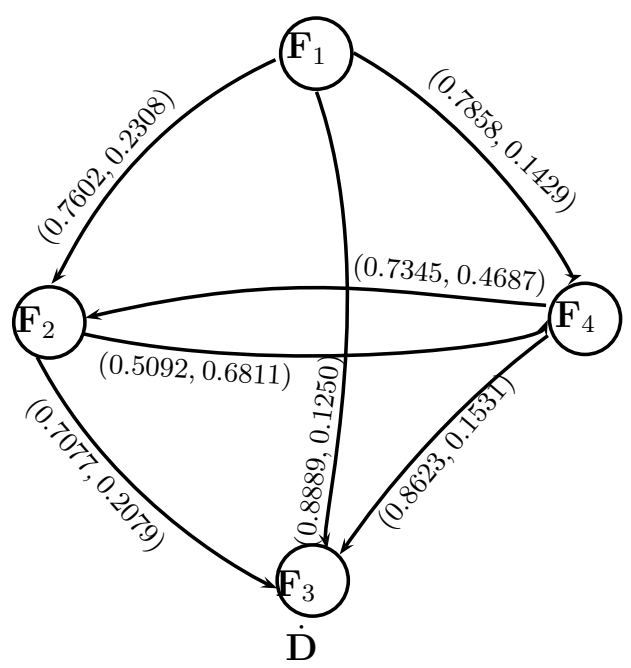

Figure 15. Partial directed network of clumped PFPR.

The out-degrees out-d $\left(\mathbf{F}_{l}\right)(l=1,2,3,4)$ of all textile industries in the partial graph are calculated as:

$$
\begin{gathered}
\text { out }-\mathrm{d}\left(\mathbf{F}_{1}\right)=(2.4349,0.4987), \text { out }-\mathrm{d}\left(\mathbf{F}_{2}\right)=(1.2169,0.8890), \\
\text { out }-\mathrm{d}\left(\mathbf{F}_{2}\right)=(0,0), \text { out }-\mathrm{d}\left(\mathbf{F}_{4}\right)=(1.5968,0.6218) .
\end{gathered}
$$

According to the membership value of out-degrees out-d $\left(\mathbf{F}_{l}\right)(l=1,2,3,4)$, we get the optimal ranking order of the four leading textile industries $\mathbf{F}_{l}$ as:

$$
\mathbf{F}_{1} \succ \mathbf{F}_{4} \succ \mathbf{F}_{2} \succ \mathbf{F}_{3}
$$

On the basis of ranking, we conclude that $\mathbf{F}_{1}$ is the most profitable textile industry among all. If the geometric averaging (PDFWGA) operator [47] is utilized in place of the PDFWAA operator, then the clumped PFN $q_{l p}=\left(\mu_{l p}, v_{l p}\right)(l, p=1,2,3,4)$ of the textile industry $\mathbf{F}_{l}$ over textile industry $\mathbf{F}_{p}$ regarding all considered criteria $\mathbf{C}^{(g)}(g=1,2,3,4$, is obtained by using Equation (2).

$$
\begin{aligned}
& q_{l p}=\operatorname{PDFWGA}\left(q_{l p}^{(1)}, q_{l p}^{(2)}, \ldots, q_{l p}^{(n)}\right) \\
& =\left(\frac{1}{1+\left[\sum_{g=1}^{n} \mathbf{W}_{g}\left(\frac{1-\mu_{l p}^{(g)}}{\mu_{l p}^{(g)}}\right)^{\gamma}\right]^{\frac{1}{\gamma}}}, \sqrt{\left.1-\frac{1}{1+\left[\sum_{g=1}^{n} \mathbf{W}_{g}\left(\frac{\left(v_{l p}^{(g)}\right)^{2}}{1-\left(v_{l p}^{(g)}\right)^{2}}\right)^{\gamma}\right]^{\frac{1}{\gamma}}}\right)} .\right.
\end{aligned}
$$

For $\gamma=1$, the corresponding clumped PFPR $\mathbf{Q}=\left(q_{l p}\right)_{4 \times 4}$ is represented in Table 6 .

Table 6. Clumped Pythagorean fuzzy preference relation.

\begin{tabular}{ccccc}
\hline $\mathbf{Q}$ & $\mathbf{F}_{1}$ & $\mathbf{F}_{2}$ & $\mathbf{F}_{3}$ & $\mathbf{F}_{4}$ \\
\hline $\mathbf{F}_{1}$ & $(0.5000,0.5000)$ & $(0.7467,0.2463)$ & $(0.8781,0.1490)$ & $(0.7778,0.1682)$ \\
$\mathbf{F}_{2}$ & $(0.2308,0.7602)$ & $(0.5000,0.5000)$ & $(0.6264,0.4481)$ & $(0.4687,0.7345)$ \\
$\mathbf{F}_{3}$ & $(0.1250,0.8889)$ & $(0.2079,0.7077)$ & $(0.5000,0.5000)$ & $(0.1531,0.8623)$ \\
$\mathbf{F}_{4}$ & $(0.1429,0.7858)$ & $(0.6811,0.5092)$ & $(0.8182,0.2688)$ & $(0.5000,0.5000)$ \\
\hline
\end{tabular}

The PFDN D, concerning clumped PFPR Q, is drawn in Figure 16. 


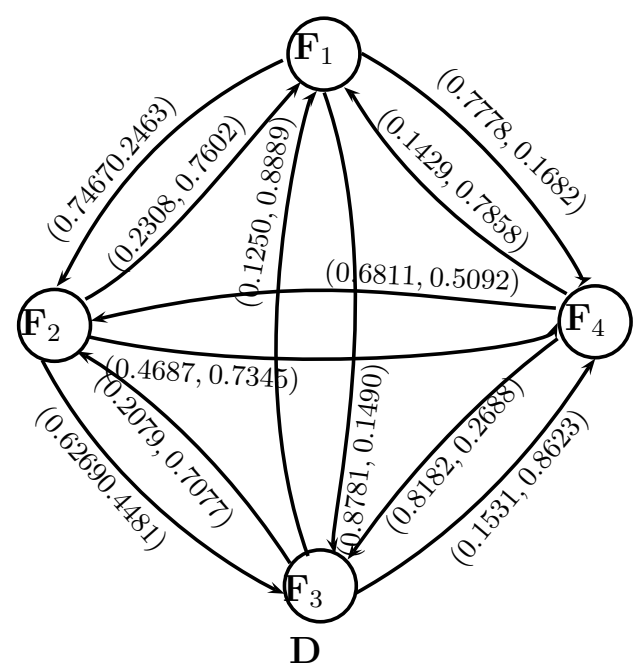

Figure 16. Directed network of clumped PFPR.

Under the condition $\mu_{l p} \geq 0.5(l, p=1,2,3,4)$, a partial directed network $\dot{\mathbf{D}}$ is drawn in Figure 17 .

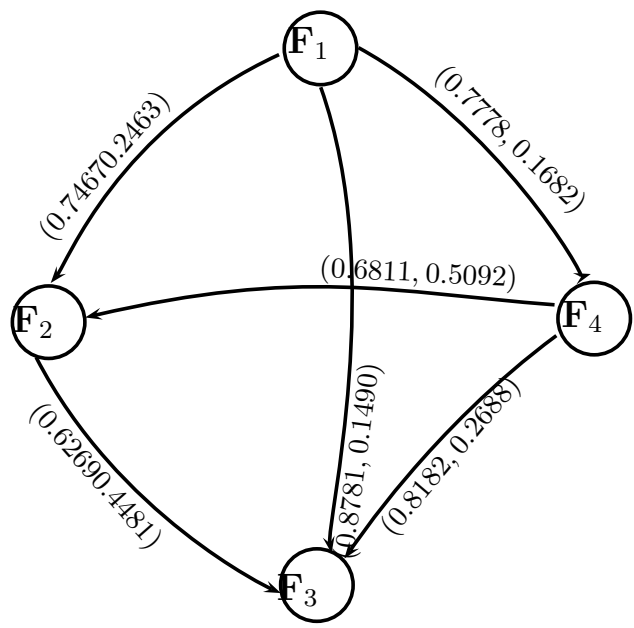

$\dot{\mathrm{D}}$

Figure 17. Partial directed network of clumped PFPR.

The out-degrees out-d $\left(\mathbf{F}_{l}\right)(l=1,2,3,4)$ of all textile industries in the partial graph are calculated as:

$$
\begin{aligned}
& \text { out }-\mathrm{d}\left(\mathbf{F}_{1}\right)=(2.4026,0.5635), \text { out }-\mathrm{d}\left(\mathbf{F}_{2}\right)=(0,0), \\
& \text { out }-\mathrm{d}\left(\mathbf{F}_{3}\right)=(0,0), \text { out }-\mathrm{d}\left(\mathbf{F}_{4}\right)=(1.4993,0.7780) .
\end{aligned}
$$

According to the membership value of out-degrees out- $\mathrm{d}\left(\mathbf{F}_{l}\right)(l=1,2,3,4)$, we get the optimal ranking order of the four leading textile industries $\mathbf{F}_{l}$ as:

$$
\mathbf{F}_{1} \succ \mathbf{F}_{4} \succ \mathbf{F}_{2} \sim \mathbf{F}_{3} .
$$

On the basis of ranking, we conclude that $\mathbf{F}_{1}$ is the most profitable textile industry among all.

Our proposed technique for multi-criteria decision making is displayed in the following Algorithm 1. 


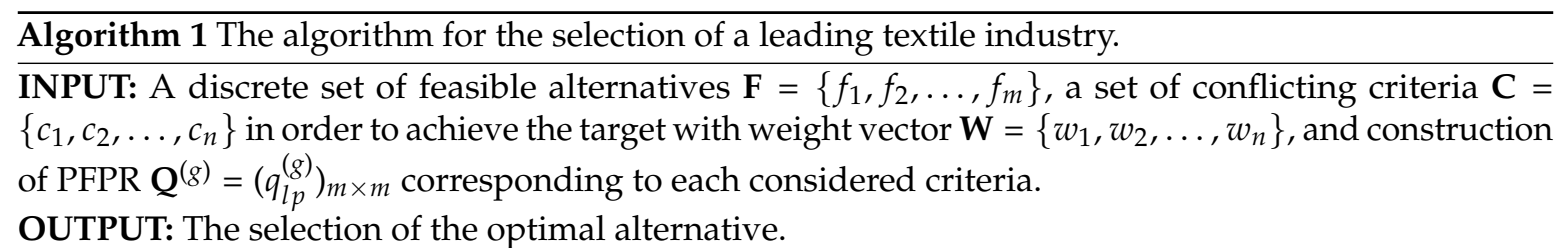

1. begin

2. Aggregate all $q_{l p}^{(g)}=\left(\mu_{l p}^{(g)}, v_{l p}^{(g)}\right)(l, p=1,2, \ldots, m)$ regarding criteria $\mathbf{C}_{g}(g=1,2,3,4)$, and get the PFPR $\mathbf{Q}=\left(q_{l p}\right)_{m \times m}$, where $q_{l p}=\left(\mu_{l p}, v_{l p}\right)$ is the PFE of the alternative $f_{l}$ over the alternative $f_{p}$ with respect to all the considered criteria $\mathbf{C}_{g}$ by using the PDFWAA operator:

$$
\begin{aligned}
q_{l p} & =\operatorname{PDFWAA}\left(q_{l p}^{(1)}, q_{l p}^{(2)}, \ldots, q_{l p}^{(n)}\right) \\
& =\left(\sqrt{1-\frac{1}{1+\left[\sum_{g=1}^{n} \mathbf{W}_{g}\left(\frac{\left(\mu_{l p}^{(g)}\right)^{2}}{1-\left(\mu_{l p}^{(g)}\right)^{2}}\right)^{\gamma}\right]^{\frac{1}{\gamma}}}}, \frac{1}{1+\left[\sum_{g=1}^{n} \mathbf{w}_{g}\left(\frac{1-v_{l p}^{(g)}}{v_{l p}^{(g)}}\right)^{\gamma}\right]^{\frac{1}{\gamma}}}\right), l, p=1,2,3, \ldots, m .
\end{aligned}
$$

or the PDFWGA operator:

$$
\begin{aligned}
q_{l p} & =\operatorname{PDFWGA}\left(q_{l p}^{(1)}, q_{l p}^{(2)}, \ldots, q_{l p}^{(n)}\right) \\
& =\left(\frac{1}{1+\left[\sum_{g=1}^{n} \mathbf{w}_{g}\left(\frac{1-\mu_{l p}^{(g)}}{\mu_{l p}^{(g)}}\right)^{\gamma}\right]^{\frac{1}{\gamma}}}, \sqrt{1-\frac{1}{1+\left[\sum_{g=1}^{n} \mathbf{W}_{g}\left(\frac{\left(v_{l p}^{(g)}\right)^{2}}{1-\left(v_{l p}^{(g)}\right)^{2}}\right)^{\gamma}\right]^{\frac{1}{\gamma}}}}\right), l, p=1,2,3, \ldots, m .
\end{aligned}
$$

3. Draw the PFDN D, regarding the aggregated PFPR $\mathbf{Q}$.

4. Under the condition $\mu_{l p} \geq 0.5(l, p=1,2, \ldots, m)$, draw the Pythagorean fuzzy partial directed graph $\dot{\mathrm{D}}$.

5. Calculate the out degrees out- $\mathrm{d}\left(f_{l}\right)(l=1,2, \ldots, m)$ of all the alternatives $f_{l}$ in the Pythagorean fuzzy partial directed graph $\dot{\mathbf{D}}$.

6. Put alternatives $f_{l}(l=1,2, \ldots, m)$ in order with regard to decreasing values of the membership degrees of out- $\mathrm{d}\left(f_{l}\right)$.

7. Alternative with the maximum membership degree of out- $\mathrm{d}\left(f_{l}\right)$ is the optimal alternative.

8. end

Algorithm 1 is also presented by a flowchart (Figure 18) for better understanding of the technique. 


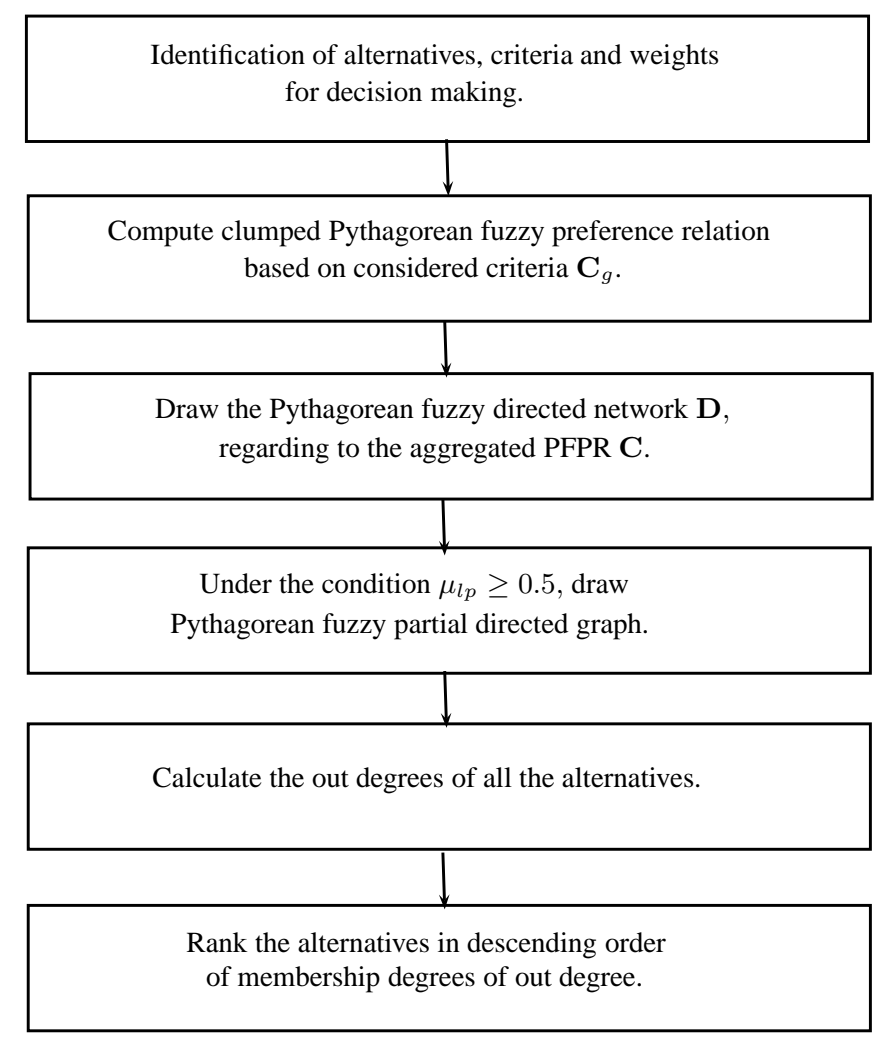

Figure 18. Flowchart of the proposed technique.

One can see that the ranking in Section 3.1 is with respect to a particular value of operational parameter $\gamma=1$. For distinct values of operational parameter $\gamma$, the stability of the ranking methodology may be noted and studied by considering a simulation study over the variety of values of the operational parameter depending on the needs.

\subsection{Comparative Analysis}

In this subsection, a comparative analysis is made between the newly proposed and exiting MCDM techniques. The Pythagorean fuzzy weighted average (PFWA) operator and the Pythagorean fuzzy weighted geometric (PFWG) operator introduced by Yager [16] are applied for solving the above decision-making problem. The ranking based on the techniques used is given in the following Table 7 .

Table 7. Different techniques for the decision results of the alternatives. PFWA, Pythagorean fuzzy weighted average; PFWG, Pythagorean fuzzy weighted geometric operator; PDFWAA, Pythagorean Dombi fuzzy weighted arithmetic averaging operator; PDFWGA, Pythagorean Dombi fuzzy weighted geometric averaging operator.

\begin{tabular}{cc}
\hline Techniques & Ranking of Alternatives \\
\hline Existing PFWA Technique [16] & $\mathbf{F}_{1} \succ \mathbf{F}_{4} \succ \mathbf{F}_{2} \succ \mathbf{F}_{3}$ \\
Existing PFWG Technique [16] & $\mathbf{F}_{1} \succ \mathbf{F}_{4} \succ \mathbf{F}_{2} \succ \mathbf{F}_{3}$ \\
Our Proposed PDFWAA Technique & $\mathbf{F}_{1} \succ \mathbf{F}_{4} \succ \mathbf{F}_{2} \succ \mathbf{F}_{3}$ \\
Our Proposed PDFWGA Technique & $\mathbf{F}_{1} \succ \mathbf{F}_{4} \succ \mathbf{F}_{2} \succ \mathbf{F}_{3}$ \\
\hline
\end{tabular}

Furthermore, Table 7 exhibits that the decision results based on the existing PFWA and PFWG techniques were consistent with our proposed PDFWAA and PDFWGA technique, which depicts the reliability of the technique. 


\section{Conclusions}

Graph models are extensively found everywhere in natural and human made structures such as process dynamics in physical, biological, and social systems and for modeling relations. PF models are more versatile and practical. It is seen that restrictions $0 \leq \mu \leq 1,0 \leq \mu+\nu \leq 1$ on FG and IFG, respectively, confine the area of these graphs to describe uncertain information that appears in the real world. PFGs with a constraint $0 \leq \mu^{2}+v^{2} \leq 1$, a generalized form, have extra spaces between membership and non-membership grades. This model gives more compatibility and precision to the system as compared to the fuzzy and intuitionistic fuzzy models. In this research article, the excellent flexibility of operational parameter $\lambda$ of Dombi operators in graph theoretical conceptions under the PF environment was observed. As the graph product is a technique that merges two graphs and produces a unique graph, hence on the basis of this, some basic graph products, in particular the direct product, semi-strong product, Cartesian product, strong product, and composition, for unifying two PDFGs, were introduced. By utilizing these products, various kinds of structural forms and models could be fused to provide a better one. For the organization process of space structures, these products may be very helpful. Further, we showed that the Cartesian product, strong product, semi-strong product, and the composition of two PDFGs were not PDFGs. Despite that if these graph products had crisp vertices, they were PDFGs. Meanwhile, these outcomes were taken on PDFGs maintaining the strongness property. Many decision-making situations can be easily solved by considering a variety of values of operational parameters. An incentive approach towards a decision-making problem related to the selection of a leading textile industry was adopted in our work. We hope this paper will help researchers to see the field of PDFGs at a glance. For further research, the vertex and edge regularity of these graph operations can be discussed.

Author Contributions: Investigation, M.A., J.M.D. and S.S.; writing-original draft, M.A. and J.M.D.; writing-review and editing, S.S.

Conflicts of Interest: The authors declare no conflict of interest.

\section{References}

1. Zadeh, L.A. Fuzzy sets. Inf. Control 1965, 8, 338-353. [CrossRef]

2. Hamacher, H. On Logical Aggregations of Non-Binar Explicit Decision Criteria; Rita, G., Ed.; Fischer Verlag: Frankfurt, Germany, 1978.

3. Kuwagaki, A. Sur l'équation fonctionnelle $f(x+y)=R\{f(x), f(y)\}$. Mem. Coll. Sci. Univ. Kyoto Ser. A Math. 1950, 26, 139-144. Available online: https://projecteuclid.org/download/pdf_1/euclid.kjm/1250777986 (accessed on 13 December 2019). [CrossRef]

4. Menger, K. Statistical metrics. J. Natl. Acad. Sci. 1942, 28, 535-537. [CrossRef] [PubMed]

5. Schweizer, B.; Sklar, S. Probabilistic Metric Spaces; Elsevier: Amsterdam, The Netherlands, 1983.

6. Alsina, C.; Trillas, E.; Valverde, L. On some logical connectives for fuzzy sets theory. J. Math. Anal. Appl. 1983, 93, 15-26. [CrossRef]

7. Klement, P.E.; Mesiar, R.; Pap, E. Triangular Norms; Kluwer Academic: Dordrecht, The Netherlands, 2000.

8. Hamacher, H. Logical combinations of fuzzy statements and their relative valuation functions. Cybern. Syst. Res. 1978, 3, 276-288.

9. Dubois, D.; Ostasiewicz, W.; Prade, H. Fuzzy Sets: History and Basic Notions; Springer: Boston, MA, USA, 2000.

10. Rosenfeld, A. Fuzzy graphs. In Fuzzy Sets and Their Applications to Cognitive and Decision Processes; Zadeh, L.A., Fu, K.S., Shimura, M., Eds.; Academic Press: Cambridge, MA, USA, 1975; pp. 77-95.

11. Mordeson, J.N.; Peng, C.S. Operations on fuzzy graphs. Inf. Sci. 1994, 79, 159-170. [CrossRef]

12. Atanassov, K.T. Intuitionistic fuzzy sets. Fuzzy Sets Syst. 1986, 20, 87-96. [CrossRef]

13. Shannon, A.; Atanassov, K.T. A first step to a theory of intuitioistic fuzzy graphs. In Proceedings of the First Workshop on Fuzzy Based Expert Systems, Sofia, Bulgaria, 28-30 September 1994; pp. 59-61.

14. Yager, R.R. Pythagorean fuzzy subsets. In Proceedings of the Joint IFSA World Congress and NAFIPS Annual Meeting, Edmonton, AB, Canada, 24-28 June 2013; pp. 57-61. 
15. Yager, R.R.; Abbasov, A.M. Pythagorean membership grades, complex numbers and decision making. Int. J. Intell. Syst. 2013, 28, 436-452. [CrossRef]

16. Yager, R.R. Pythagorean membership grades in multi-criteria decision making. IEEE Trans. Fuzzy Syst. 2014, 22, 958-965. [CrossRef]

17. Zhang, X.; $\mathrm{Xu}, \mathrm{Z}$. Extension of TOPSIS to multiple criteria decision making with Pythagorean fuzzy sets. Int. J. Intell. Syst. 2014, 29, 1061-1078. [CrossRef]

18. Peng, X.; Yang, Y. Some results for Pythagorean fuzzy sets. Int. J. Intell. Syst. 2015, 30, 1133-1160. [CrossRef]

19. Akram, M.; Dudek, W.A.; Ilyas, F. Group decision-making based on Pythagorean fuzzy TOPSIS method. Int. J. Intell. Syst. 2019. [CrossRef]

20. Akram, M.; Ilyas, F.; Garg, H. Multi-criteria group decision making based on ELECTRIC I method in Pythagorean fuzzy information. Soft Comput. 2019. [CrossRef]

21. Rangasamy, P.; Palaniappan, N. Some operations on intuitionistic fuzzy sets of second type. Notes Intuitionistic Fuzzy Sets 2003, 10, 1-19.

22. Ren, P.; Xu, Z.; Gou, X. Pythagorean fuzzy TODIM approach to multi-criteria decision making. Appl. Soft Comput. 2016, 42, 246-259. [CrossRef]

23. Peng, X.; Selvachandran, G. Pythagorean fuzzy set: State of the art and future directions. Artifical Intell. Rev. 2017. [CrossRef]

24. Garg, H. A new exponential operational laws and their aggregation operators of interval-valued Pythagorean fuzzy information. Int. J. Intell. Syst. 2018, 33, 653-683. [CrossRef]

25. Garg, H. Some methods for strategic decision-making problems with immediate probabilities in Pythagorean fuzzy environment. Int. J. Intell. Syst. 2018, 33, 687-712. [CrossRef]

26. Garg, H. Linguistic Pythagorean fuzzy sets and its applications in multi attribute decision making process. Int. J. Intell. Syst. 2018, 33, 1234-1263. [CrossRef]

27. Garg, H. Hesitant Pythagorean fuzzy sets and their aggregation operators in multiple-attribute decision-making. Int. J. Uncertain. Quantif. 2018, 8, 267-289. [CrossRef]

28. Garg, H. A novel correlation coefficients between Pythagorean fuzzy sets and its applications to decision-making processes. Int. J. Intell. Syst. 2016, 31, 1234-1253. [CrossRef]

29. Naz, S.; Ashraf, S.; Akram, M. A novel approach to decision-making with Pythagorean fuzzy information. Mathematics 2018, 6, 95. [CrossRef]

30. Verma, R.; Merigo, J.M.; Sahni, M. Pythagorean fuzzy graphs: Some results. arXiv 2018, arXiv:1806.06721v1.

31. Akram, M.; Naz, S. Energy of Pythagorean fuzzy graphs with applications. Mathematics 2018, 6, 136. [CrossRef]

32. Akram, M.; Habib, A.; Ilyas, F.; Dar, J.M. Specific types of Pythagorean fyzzy graphs and application to decision-making. Math. Comput. Appl. 2018, 23. [CrossRef]

33. Akram, M.; Dar, J.M.; Naz, S. Certain graphs under Pythagorean fuzzy environment. Complex Intell. Syst. 2019, 5, 127-144. [CrossRef]

34. Akram, M.; Dar, J.M.; Farooq, A. Planar graphs under Pythagorean fuzzy environment. Mathematics $2018,6$. [CrossRef]

35. Akram, M.; Ilyas, F.; Saeid, A.B. Certain notions of Pythagorean fuzzy graphs. J. Intell. Fuzzy Syst. 2019, 36, 5857-5874. [CrossRef]

36. Naz, S.; Rashmanlou, H.; Malik, M.A. Operations on single-valued neutrosophic graphs with application. J. Intell. Fuzzy Syst. 2017, 32, 2137-2151. [CrossRef]

37. Akram, M.; Habib, A. $q$-Rung picture fuzzy graphs: A creative view on regularity with applications. J. Appl. Math. Comput. 2019, 61, 235-280. [CrossRef]

38. Habib, A.; Akram, M.; Farooq, A. q-Rung orthopair fuzzy competition graphs with application in the soil ecosystem. Mathematics 2019, 7. [CrossRef]

39. Akram, M.; Habib, A.; Koam, A.N. A novel description on edge-regular $q$-rung picture fuzzy graphs with application. Symmetry 2019, 11, 489. [CrossRef]

40. Dombi, J. A general class of fuzzy operators, the DeMorgan class of fuzzy operators and fuzziness measures induced by fuzzy operators. Fuzzy Sets Syst. 1982, 8, 149-163. [CrossRef]

41. Dombi, J. Towards a general class of operators for fuzzy systems. IEEE Trans. Fuzzy Syst. 2008, 16, 477-484. [CrossRef] 
42. Chen, J.; Ye, J. Some single-valued neutrosophic Dombi weighted aggregation operators for multiple attribute decision-making. Symmetry 2017, 9. [CrossRef]

43. Shi, L.; Ye, J. Dombi aggregation operators of neutrosophic cubic sets for multiple attribute decision-making. Algorithms 2018, 11. [CrossRef]

44. Jana, C.; Pal, M.; Wang, J. Bipolar fuzzy Dombi aggregation operators and its application in multiple-attribute decision-making process. J. Ambient Intell. Humaniz. Comput. 2018. [CrossRef]

45. Liu, P.D.; Liu, J.L.; Chen, S.M. Some intuitionistic fuzzy Dombi Bonferroni mean operators and their application to multi-attribute group decision making. J. Oper. Res. Soc. 2018, 69, 1-24. [CrossRef]

46. He, X. Typhoon disaster assessment based on Dombi hesitant fuzzy information aggregation operators. Nat. Hazards 2018, 90, 1153-1175. [CrossRef]

47. Akram, M.; Dudek, W.A.; Dar, J.M. Pythagorean Dombi fuzzy aggregation operators with application in multi-criteria decision-making. Int. J. Intell. Syst. 2019. [CrossRef]

48. Ashraf, S.; Naz, S.; Kerre, E.E. Dombi fuzzy graphs. Fuzzy Inf. Eng. 2018, 10, 58-79. [CrossRef]

49. Akram, M.; Dar, J.M.; Naz, S. Pythagorean Dombi fuzzy graphs. Complex Intell. Syst. 2019, 1-26. [CrossRef]

(C) 2019 by the authors. Licensee MDPI, Basel, Switzerland. This article is an open access article distributed under the terms and conditions of the Creative Commons Attribution (CC BY) license (http://creativecommons.org/licenses/by/4.0/). 